\title{
Amelioration of Alzheimer's disease pathology by mitophagy inducers identified via machine learning and a cross-species workflow
}

\author{
Chenglong Xie ${ }^{1,2,3,4,5,21}$, Xu-Xu Zhuang ${ }^{6,21}$, Zhangming Niu ${ }^{7,8,21}$, Ruixue Ai ${ }^{2,21}$, Sofie Lautrup ${ }^{2}$, \\ Shuangjia Zheng ${ }^{9}$, Yinghui Jiang ${ }^{8}$, Ruiyu Han², Tanima Sen Gupta², Shuqin Cao², \\ Maria Jose Lagartos-Donate ${ }^{2}$, Cui-Zan Cai ${ }^{6}$, Li-Ming $\mathrm{Xie}^{6}$, Domenica Caponio (D) ${ }^{2}$, \\ Wen-Wen Wang ${ }^{10}$, Tomas Schmauck-Medina ${ }^{2}{ }^{2}$, Jianying Zhang ${ }^{2}$, He-ling Wang ${ }^{2}{ }^{2}$, Guofeng Lou ${ }^{2}$, \\ Xianglu Xiao ${ }^{8}$, Wenhua Zheng ${ }^{11}$, Konstantinos Palikaras ${ }^{12}$, Guang Yang ${ }^{13,14}$, Kim A. Caldwell ${ }^{15,16}$, \\ Guy A. Caldwell ${ }^{15,16}$, Han-Ming Shen ${ }^{17,18}$, Hilde Nilsen ${ }^{2,19}$, Jia-Hong Lu ${ }^{6 \bowtie}$ and Evandro F. Fang ${ }^{2,19,20 凶}$
}

A reduced removal of dysfunctional mitochondria is common to aging and age-related neurodegenerative pathologies such as Alzheimer's disease (AD). Strategies for treating such impaired mitophagy would benefit from the identification of mitophagy modulators. Here we report the combined use of unsupervised machine learning (involving vector representations of molecular structures, pharmacophore fingerprinting and conformer fingerprinting) and a cross-species approach for the screening and experimental validation of new mitophagy-inducing compounds. From a library of naturally occurring compounds, the workflow allowed us to identify 18 small molecules, and among them two potent mitophagy inducers (Kaempferol and Rhapontigenin). In nematode and rodent models of $A D$, we show that both mitophagy inducers increased the survival and functionality of glutamatergic and cholinergic neurons, abrogated amyloid- $\beta$ and tau pathologies, and improved the animals' memory. Our findings suggest the existence of a conserved mechanism of memory loss across the AD models, this mechanism being mediated by defective mitophagy. The computational-experimental screening and validation workflow might help uncover potent mitophagy modulators that stimulate neuronal health and brain homeostasis.

A ccumulation of damaged mitochondria in the brain is a hallmark of brain aging and related neurodegenerative diseases, including Alzheimer's disease $(\mathrm{AD})^{1-5}$. As the most common form of dementia, $\mathrm{AD}$ affects around 50 million individuals worldwide without an available cure ${ }^{6}$. Accumulation of amyloid $\beta$ (A $\beta)$ and neurofibrillary tangles (majorly $\mathrm{p}$-Tau aggregates) are the disease-defining pathological features of AD. However, clinical drug developments targeting $\mathrm{A} \beta$ and Tau have struggled to produce positive results ${ }^{7}$, highlighting the urgent need for discovery and development of novel therapeutic interventions. Mitochondria are fundamental subcellular organelles that generate adenosine triphosphate (ATP), which is essential for the excitability and survival of neurons. In addition, they are at the centre of signalling pathways regulating $\mathrm{Ca}^{2+}$, oxidative stress, developmental and synaptic plasticity, as well as neuronal fate determination ${ }^{8}$. Mitochondria constantly experience endogenous (for example, DNA damage and oxidative toxicants) and exogenous (for example, environmental exposure) stresses, which cause structural and/or functional damage to these essential organelles ${ }^{9}$. In a normal physiological environment, damaged mitochondria are efficiently cleared by mitophagy, a subtype of selective macroautophagy (hereafter referred to as autophagy) ${ }^{10}$. However, in elderly individuals or people with common neurodegenerative diseases such as AD, Parkinson's disease, Amyotrophic lateral sclerosis and Huntington's disease in which accumulation of defective mitochondria is a common feature, and possibly a driving force of memory impairment and dementia, autophagic processes may be disrupted ${ }^{1,5,10}$. Emerging evidence highlights that mitophagy impairment mediates the accrual of

'Department of Neurology, The First Affiliated Hospital of Wenzhou Medical University, Wenzhou, China. ${ }^{2}$ Department of Clinical Molecular Biology, University of Oslo and Akershus University Hospital, Lørenskog, Norway. ${ }^{3}$ Institute of Aging, Wenzhou Medical University, Wenzhou, China. ${ }^{4}$ Oujiang Laboratory, Wenzhou, Zhejiang, China. ${ }^{5}$ Key Laboratory of Alzheimer's Disease of Zhejiang Province, Wenzhou, China. ${ }^{6}$ State Key Laboratory of Quality Research in Chinese Medicine, Institute of Chinese Medical Sciences, University of Macau, Macau, China. ${ }^{7}$ Aladdin Healthcare Technologies Ltd., London, UK. ${ }^{8}$ MindRank Al Ltd., Hangzhou, Zhejiang, China. ${ }^{9}$ School of Data and Computer Science, Sun Yat-sen University, Guangzhou, China. ${ }^{10} \mathrm{Center}$ of Traditional Chinese Medicine, The Second Affiliated Hospital and Yuying Children's Hospital of Wenzhou Medical University, Wenzhou, China. ${ }^{1} F a c u l t y$ of Health Sciences, University of Macau, Taipa, Macau, China. ${ }^{2}$ Department of Physiology, School of Medicine, National and Kapodistrian University of Athens, Athens, Greece. ${ }^{13}$ Cardiovascular Research Centre, Royal Brompton Hospital, London, UK. ${ }^{14}$ National Heart and Lung Institute, Imperial College London, London, UK. ${ }^{15}$ Department of Biological Sciences, The University of Alabama, Tuscaloosa, AL, USA. ${ }^{16}$ Departments of Neurology and Neurobiology, Center for Neurodegeneration and Experimental Therapeutics, Nathan Shock Center for Research on the Basic Biology of Aging, University of Alabama at Birmingham School of Medicine, Birmingham, AL, USA. ${ }^{17}$ Department of Physiology, Yong Loo Lin School of Medicine, National University of Singapore, Singapore, Singapore. ${ }^{18} \mathrm{Faculty}$ of Health Sciences, University of Macau, Macau, China. ${ }^{19}$ The Norwegian Centre on Healthy Ageing (NO-Age), Oslo, Norway. ${ }^{20}$ Department of Geriatrics, The First Affiliated Hospital, Zhengzhou University, Zhengzhou, China. ${ }^{21}$ These authors contributed equally: Chenglong Xie, Xu-Xu Zhuang, Zhangming Niu and Ruixue Ai.凶e-mail: jiahonglu@um.edu.mo; e.f.fang@medisin.uio.no 
dysfunctional mitochondria in the AD brain ${ }^{11}$. Indeed, the basallevels of mitophagy are less than $50 \%$ in $\mathrm{AD}$ patient brain tissue compared with healthy controls ${ }^{11}$. Moreover, several regulators of autophagy and mitophagy pathways, such as phosphatidylinositol-binding clathrin assembly protein (PICALM) ${ }^{12}$, presenilin 1 (PS1) ${ }^{13}$, phosphatase and tensin homologue (PTEN)-induced kinase1 (PINK1), TANK-binding kinase 1 (TBK1), Unc-51-like kinase-1 (ULK1) ${ }^{11}$ and $\mathrm{Bcl}-2$ associated athanogene 3 (BAG-3) ${ }^{14}$ are lowly expressed or impaired in $\mathrm{AD}$ patients. Genetic and/or pharmacologic restoration of mitophagy inhibits disease progression in preclinical AD models $^{11,15}$. Given the continued failures in anti-AD drug development, approaches targeting the broader aspects of AD pathologies, such as defective mitophagy, may hold a therapeutic potential.

Bioavailable neuronal mitophagy inducers are scarce. Thus, we set out to develop a screening workflow combining advanced artificial intelligence (AI) and classical wet laboratory approaches to identify novel mitophagy modulators as potential drug candidates for $\mathrm{AD}$ treatment. The application of traditional chemistry or high-throughput approaches for drug discovery is time-consuming and also carry a high failure rate ${ }^{16}$. Machine learning is emerging as a powerful, fast, reliable and cost-effective approach to drug development, which can accelerate discovery and decision making for predefined questions with precise data ${ }^{17-20}$. Machine learning has been used in pharmaceutical development, bioactivity prediction, de novo molecular design, synthesis prediction and biological image analysis, among other applications ${ }^{19,21,22}$. It is a popular tool in drug discovery when using a large arsenal of compounds; however, the limitation of broad application is the necessity for a sizeable number of labelled data points to ensure model generalizability and avoid overfitting ${ }^{19,22}$. In view of the scarcity of known mitophagy inducers, an alternative machine learning approach is the use of 'biological fingerprints', which are representations of chemical structures originally designed to assist in chemical database substructure searching ${ }^{23}$. Here we outline the development of an AI-aided high-throughput screen workflow that combines AI, mammalian cells, nematodes and mice to create an approach for identifying potent mitophagy modulators.

\section{Results}

An AI-aided model for screening of mitophagy inducers. A combinational molecular representation approach, including Mol2vec, pharmacophore fingerprint and 3D conformers fingerprint, was used for modelling (Fig. 1a,b). We first compiled a dataset that was large-scale, structurally diverse and task related. The $\mathrm{ChEMBL}^{24}$ and ZINC natural product database ${ }^{25}$ were filtered using procedures outlined elsewhere ${ }^{26}$, producing a dataset with 19.9 million compounds (that is, the pre-training dataset). This pre-training dataset was used to train the multi-representations model, which translated a molecule into an information-enriched structure vector in an unsupervised manner, without the need for numerous annotated data. The model followed a natural language processing strategy ${ }^{26}$, wherein molecules were considered as sentences and substructures as words. By iteratively learning the relative position of each substructure in a molecule, the model could finally capture the global structural information of each substructure in the chemical space.
New molecules could be described by summing the substructure vectors retrieved from a pre-trained Mol2vec model. The obtained compound feature vectors could then be used to calculate the structural distance of any two compounds in the projected chemical space. Further, to fill in the blanks of $2 \mathrm{D}$ pharmacophore and $3 \mathrm{D}$ conformer information, the pharmacophore and shape fingerprinting techniques were introduced to augment the representation of molecules (Fig. 1a). A total of 14 known mitophagy inducers were used as reference (Supplementary Table 1).

After model development and augmentation, we subsequently applied it to identify potential mitophagy inducers from a natural product library (named Macau Library), which contained 3,274 natural compounds isolated mainly from a series of traditional Chinese medicinal plants that have been used to treat neurodegenerative diseases and other diseases ${ }^{27,28}$. Similarity scores for each compound against each of the 14 known mitophagy inducers were determined, compounds were ranked on the basis of their structural (Mol2vec score), pharmacophore fingerprint and shape distance (3D conformers fingerprint) against the known inducers (Supplementary Table 1). We identified a total of 18 molecules in the Macau Library that were most similar to the existing (known) mitophagy inducers, with a threshold of 0.75 . We set the threshold to 0.75 on the basis of published works ${ }^{29,30}$ and our in-house justification of the workload for wet-lab validation. Detailed information of the 18 in silico-selected molecules is documented (Supplementary Fig. 1 and Table 2). We further performed chemical similarity analysis of the top 18 molecules: while 1D similarities are lower than $40 \%$ between any two compounds, there are some compounds with high scores in 2D (for example, 91\% for T2174 and T0579) and 3D (for example, 71\% for T3S1068 and T2177) similarity analyses (Additional Supplementary Table). More details on the AI procedures can be found in Methods.

In vitro and in vivo validation of mitophagy candidates. The 18 AI-selected molecules were then subjected to experimental verification in both human cells (HeLa cells) and the soil-dwelling nematode Caenorhabditis elegans. HeLa cells co-expressing the E3 ubiquitin ligase Parkin and the mitochondria-targeted form of monomeric Keima fluorescent reporter $(\mathrm{mt}-\mathrm{Keima})^{31}$ were used. Keima is a coral-derived, lysosomal degradation-resistant, dual-excitation ratio-metric fluorescent protein that is $\mathrm{pH}$-sensitive; it shows shorter-wavelength excitation (green) in healthy mitochondria normally with neutral $\mathrm{pH}$, while it turns to longer-wavelength excitation (red) in damaged mitochondria undergoing acidic lysosomal degradation (Fig. $2 \mathrm{a})^{31}$. These features of the mt-Keima reporter allow qualitative assessment of mitophagic flux in both cells and mouse model ${ }^{31-33}$. To ensure high translational potential, we started with a series of doses covering $0.1,1.0$ and $10 \mu \mathrm{M}$, with $10 \mu \mathrm{M}$ as the cut-off threshold. In a first-pass study of the 18 AI-selected compounds, 8 molecules (Quercetin (Macau Library ID: T-2174), Quercetin dihydrate (T-6630), Tacrolimus (T-2144), Ascomycin (T-2481), Isorhamnetin (T-2836), Pinostilbene (T-3755), Kaempferol (Kaem, T-2177) and Rhapontigenin (Rhap, T-3776)) induced mitophagy at $10 \mu \mathrm{M}$; the remaining 10 molecules did not induce detectable mitophagy up to $10 \mu \mathrm{M}$ and were excluded from the study at this

Fig. 1 | The use of combined machine learning strategies to identify novel mitophagy inducers. a, The workflow for model pre-training: (i) Molecules within the pre-training dataset were transferred into SMILES sequences, molecular interaction features and 3D conformers fingerprint in the data preparation stage; (ii) Three encoders (for 1D, 2D and 3D representations) were then designed to embed the input data, and these representational embeddings were aggregated into the encoder model of the multi-representation; (iii) The multi-representational embeddings were then passed to the representation decoder to pre-train the multi-representation molecule model. ' $F$ ' and ' $G$ ' stand for 'Functional encoder' and 'Generator' respectively. b, The workflow for the virtual screening process: (i) The virtual screening library contained 3,274 molecules from a traditional Chinese medicine dataset, named Macau Library; (ii) The 1D, 2D and 3D molecular representations for each compound were generated on the basis of the pre-trained molecule representation models; (iii) The representations were then aggregated and clustered, and a hyper-space filter was applied to the representations to filter out outliers; (iv) The similarity scores for each compound were calculated to generate the top $\mathrm{N}$ candidate compounds. 
point (Fig. 2b,c and Supplementary Fig. 2a,c,d). It was noted that Quercetin dihydrate and Quercetin exhibited very similar results, likely due to their structural and functional similarities, thus Quercetin dihydrate was eliminated from the study at this point. To confirm whether the 7 remaining molecules trigger mitophagy in a dose-dependent manner, we administered higher doses of each compound $(20,50$ and $100 \mu \mathrm{M})$ to the same HeLa mt-Keima cells.
We were unable to observe any dose-dependent mitophagic upregulation in response to Quercetin, Tacrolimus and Ascomycin supplementation past $10 \mu \mathrm{M}$. However, Isorhamnetin, Pinostilbene, Kaem and Rhap administration triggered mitophagy in a dose-dependent manner (Fig. 2b,c). Therefore collectively, among the 18 AI-selected molecules, 8 showed an ability to stimulate mitophagy in vitro, with 4 of them inducing mitophagy in a dose-dependent manner.
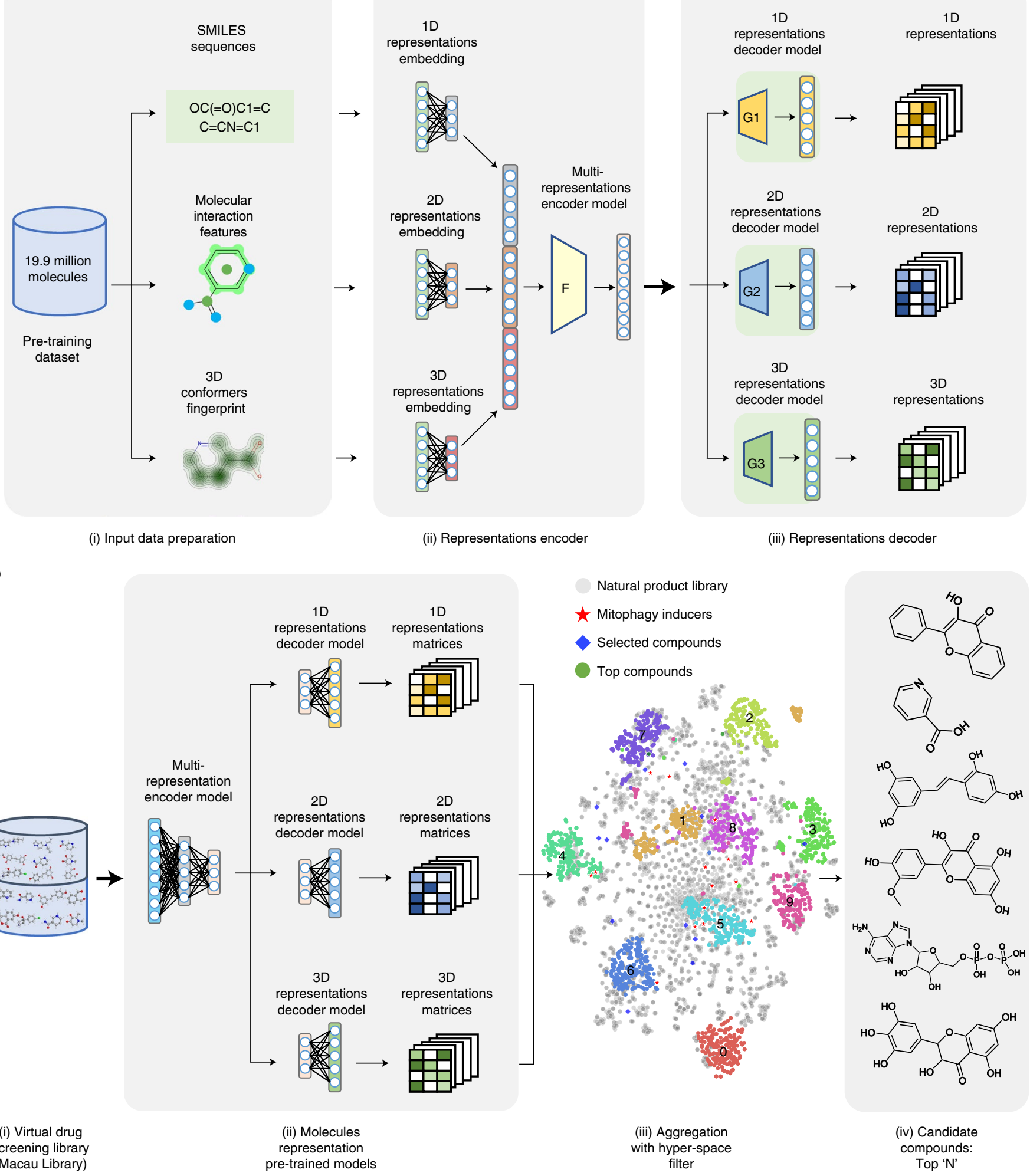
To investigate whether the aforementioned mitophagy inducers could mediate neuronal mitophagy in vivo, we used transgenic nematodes with pan-neuronal expression of mitochondria-targeted Rosella (mt-Rosella, a dual colour-emission biosensor). The mt-Rosella biosensor comprises a green fluorescent protein (GFP) variant sensitive to the acidic environment of the lysosomal lumen, which is fused to the fast-maturing $\mathrm{pH}$-insensitive DsRed. Mitophagy index is assessed by monitoring the GFP/DsRed ratio, with reduced values signifying mitophagy induction ${ }^{11}$ (Supplementary Fig. 4a). We supplemented $0.2 \mathrm{mM}$ and $1.0 \mathrm{mM}$ of each mitophagy-inducing compound to mt-Rosella-expressing animals from eggs onwards and analysed mitophagy levels in 1-day-old adults. Rotenone, a mitochondrial complex I inhibitor, was used as positive control to trigger mitophagy. Quercetin (at $1 \mathrm{mM}$ ), Kaem (at $0.2 \mathrm{mM}$ ) and Rhap (at both $0.2 \mathrm{mM}$ and $1 \mathrm{mM}$ ) were able to induce neuronal mitophagy in worms, while Tacrolimus, Ascomycin, Isorhamnetin and Pinostilbene were negative for neuronal mitophagy induction (Fig. 2d and Supplementary Fig. 4b). In summary, among the 18 AI-selected candidates, 3 - Quercetin, Kaem and Rhap - stimulated mitophagy in both human cells and C. elegans neurons.

In addition to using $\mathrm{HeLa}$ mt-Keima cells and mt-Rosella-expressing animals to quantify mitophagy by Kaem and Rhap, we further validated the robust mitophagy induction capacities of Kaem and Rhap. Firstly, immunoblot data indicate that both Kaem and Rhap dose-independently (20, 40 and $80 \mu \mathrm{M}$ for $24 \mathrm{~h}$ ) reduced the expression of the mitochondrial outer membrane protein MFN2 and mitochondrial inner membrane protein Tim23 in both YFP-Parkin-expressing HeLa cells and Mito-GFPand mCherry-Parkin-expressing HeLa cells (Fig. 3a-h). Secondly, Kaem and Rhap $(20 \mu \mathrm{M}, 24 \mathrm{~h})$ enhanced co-localization of mitochondria (Mito-GFP) with the LAMP1-antibody-labelled lysosome, indicating increased lysosomal degradation of mitochondria via mitophagy (Fig. 3i,j). Thirdly, Kaem and Rhap at $0.2 \mathrm{mM}$ stimulated neuronal mitophagy in C. elegans as evidenced by increased LGG-1/Atg-8 to DCT-1/NIX co-localization, and increased mitochondria in the lysosomes as shown by reduced GFP/DsRed (Fig. $3 \mathrm{k}, 1)$. Fourthly, data from electron microscopy (EM) showed that Kaem and Rhap induced mitophagosome-like events in HeLa cells (Fig. $3 \mathrm{~m}$ and Extended Data Fig. 1a), as well as in hippocampal brain tissues from wild-type (WT) and AD-like 3xTg mice (Fig. $3 \mathrm{n}$ and Extended Data Fig. 1b; details on the mouse studies are shown below). To note, lower doses of either Kaem or Rhap were unable to induce mitophagy in HeLa mt-Keima cells $(2.5$ and $5 \mu \mathrm{M}$, 24 h; Supplementary Fig. 2b) or the nematode neurons (0.01, 0.05, $0.1 \mathrm{mM}$; Fig. $3 \mathrm{k}, 1)$. Collectively, these data unequivocally point to robust mitophagy stimulation capacity of both Kaem and Rhap in cell culture system, nematodes and mice.

Additionally, we compared our combinational AI model (Fig. 1a) with other machine learning approaches (1D, 2D or 3D) to determine their accuracy in identifying mitophagy inducers. We selected the top 5 scored compounds from each of the independent approaches for validation in HeLa mt-Keima cells. Compounds in the $1 \mathrm{D}$ - and $3 \mathrm{D}$-selected lists, at $10 \mu \mathrm{M}$, were unable to induce detectable mitophagy in HeLa mt-Keima cells (Supplementary
Fig. 3). All top 5 compounds recommended by the $2 \mathrm{D}$ approach were in the top 18 list selected by our combinational AI model: 3 compounds (T0879, T2812, T2144 at $10 \mu \mathrm{M}$ ) were unable to induce mitophagy, the remaining 2 (Ascomycin (T2481) and Pinostilbene (T3755)) were able to induce mitophagy in cells, but were unable to induce neuronal mitophagy in nematodes (Fig. 2). Additionally, we reviewed and listed the hit rate for experimental high-throughput screening and other AI drug discovery projects for a comprehensive comparison study. Overall, the hit rate of our model (in vitro validation) is higher than the experimental high-throughput screening ( $44 \%$ vs $0.14 \%)$ and substantially outperforms other machine learning, quantitative structure-activity relationship (QSAR) and computer-aided approaches (Supplementary Table 3). Collectively, these in silico, in vitro and in vivo data indicate that our combinational AI approach is more accurate in predicting molecules with mitophagy induction and neuroprotection activities both in vitro and in vivo, than the individual $1 \mathrm{D}, 2 \mathrm{D}$ or $3 \mathrm{D}$ approaches.

Kaem and Rhap inhibit memory loss in $\mathbf{A} \beta_{1-42}$ C. elegans. Recent evidence underlies the likely causative role of compromised mitophagy in $\mathrm{AD}$ pathogenesis ${ }^{11,34}$. Thus, we examined the impact of the newly identified mitophagy stimulators on memory improvement in both $A \beta$ and Tau nematode models. To investigate whether pharmacological upregulation of mitophagy restores memory deficits, we evaluated learned behaviour in transgenic nematodes, whereby they have pan-neuronal expression of human $A \beta_{1-42}\left(\mathrm{hA} \beta_{1-42}\right)^{35}$, via aversive olfactory learning chemotaxis assay (where a negative value correlates with chemotaxis-related memory ${ }^{11}$. hA $\beta_{1-42}$ nematodes treated with Kaem or Rhap displayed improved learned behavioural performance, while Quercetin did not appear to restore associative memory deficits (Fig. 4a and Supplementary Fig. 5a). Thus, among the 18 AI-selected candidates, Kaem and Rhap demonstrated the capacity to stimulate mitophagy in both human cells and C. elegans neurons, and improved an established measure of simple associative memory in these transgenic hA $\beta_{1-42}$ worms.

We then investigated the underlying molecular mechanisms to identify how Kaem and Rhap improve memory, focusing on mitophagy/autophagy-related pathways. While none of the compounds had any effect on the mRNA levels of pink-1, pdr-1 (orthologue of human PARK2/Parkin), bec-1 (orthologue of human $B E C N 1 / B e c l i n-1), v p s-34$ and $s k n-1$ (a stress response gene also involved in mitophagy ${ }^{36}$ ), they both increased transcriptional levels of $d c t-1$ and sqst-1 (SQSTM1 gene in humans) in transgenic hA $\beta_{1-42}$ worms (Fig. 4b). To consider translational and post-translational modifications, and due to limitations in available antibodies for C. elegans studies, we extended our mechanistic studies to human HeLa cells and scrutinized the mitophagy-inducing capacity of the molecules by checking them against a list of mitophagy/autophagy proteins that are known to be critical in mitochondrial metabolism or that are altered in $\mathrm{AD}^{11}$. In most cases, Kaem increased levels of PINK1, Parkin, Beclin-1, LC3B-II and AMBRA1, and reduced p62 in a dose-dependent manner; a very similar pattern was seen in the Rhap-treated human cells (Extended Data Fig. 2). Moreover, Rhap treatment increased the phosphorylation levels of the autophagy

Fig. 2 | Evaluation of mitophagy stimulation capacity of the Al top-scored molecules in vitro (mt-Keima) and in animals (mt-Rosella). a, A schematic representation showing mechanisms of how the mt-Keima protein can be used as a mitophagy reporter. For confocal microscopy, dual-excitation ratio imaging was carried out with two sequential excitation lasers ( $458 \mathrm{~nm}$ and $561 \mathrm{~nm}$ ). Representative confocal images are of HeLa cells expressing mt-Keima treated with vehicle (DMSO) or Carbonyl cyanide m-chlorophenyl hydrazone (CCCP) (15 $\mu$ M, 3 h). b,c, Effects of Quercetin, Tacrolimus, Ascomycin, Isorhamnetin, Pinostilbene, Kaem and Rhap (from $0.1 \mu \mathrm{M}$ to $100 \mu \mathrm{M}, 24 \mathrm{~h}$ ) on mitophagy induction. d, Effects of in vitro-positive mitophagy inducers on the induction of neuronal mitophagy in worms expressing mt-Rosella reporter. Rotenone ( $5 \mu \mathrm{M}$ and $10 \mu \mathrm{M}, 4 \mathrm{~h})$ was used as positive control. Data were pooled from 2 biological replicates (total $n=20-35$ nematodes per group), with results shown as mean \pm s.e.m. Two-way ANOVA followed by Tukey's multiple comparisons test; NS, no significance; ${ }^{\star} P<0.05,{ }^{\star \star} P<0.01,{ }^{\star \star \star} P<0.001$. A set of representative images of cellular positive (related to Fig. $2 \mathrm{~b}, \mathrm{c}$ ) and negative mitophagy inducers (with quantifications) is included in Supplementary Fig. 2. Mechanisms of the mt-Rosella sensor as well as a set of representative images (related to Fig. 2d) are shown in Supplementary Fig. 4. 
factor ULK1 at Ser555, which is essential for mitophagy initiation ${ }^{37}$ (Extended Data Fig. 2). In addition, to increase the expression of multiple mitophagy-related proteins, Kaem or Rhap supplementation resulted in reduced protein levels of Mitofusin-2 (MFN2) and elevated the ratio of p-DRP1 (Ser616)/DRP1 (Extended Data Fig. 2). These findings suggest that mitochondrial fission is a

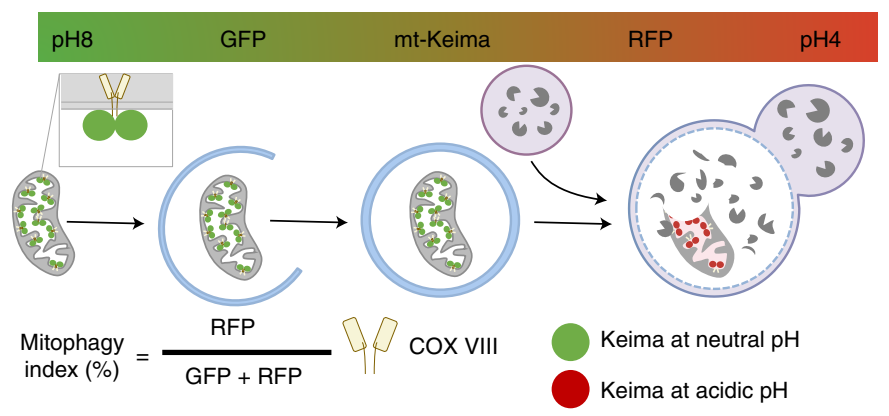

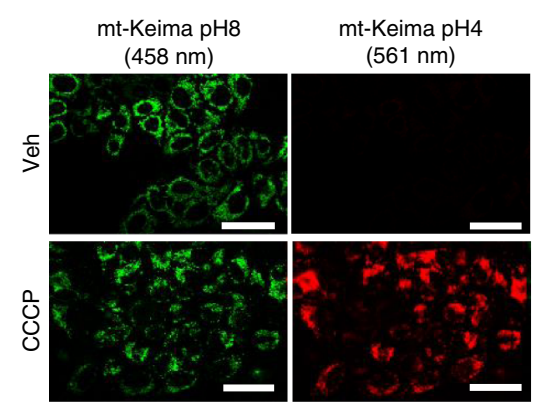

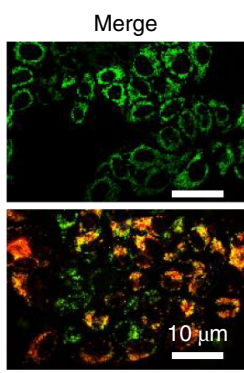

b
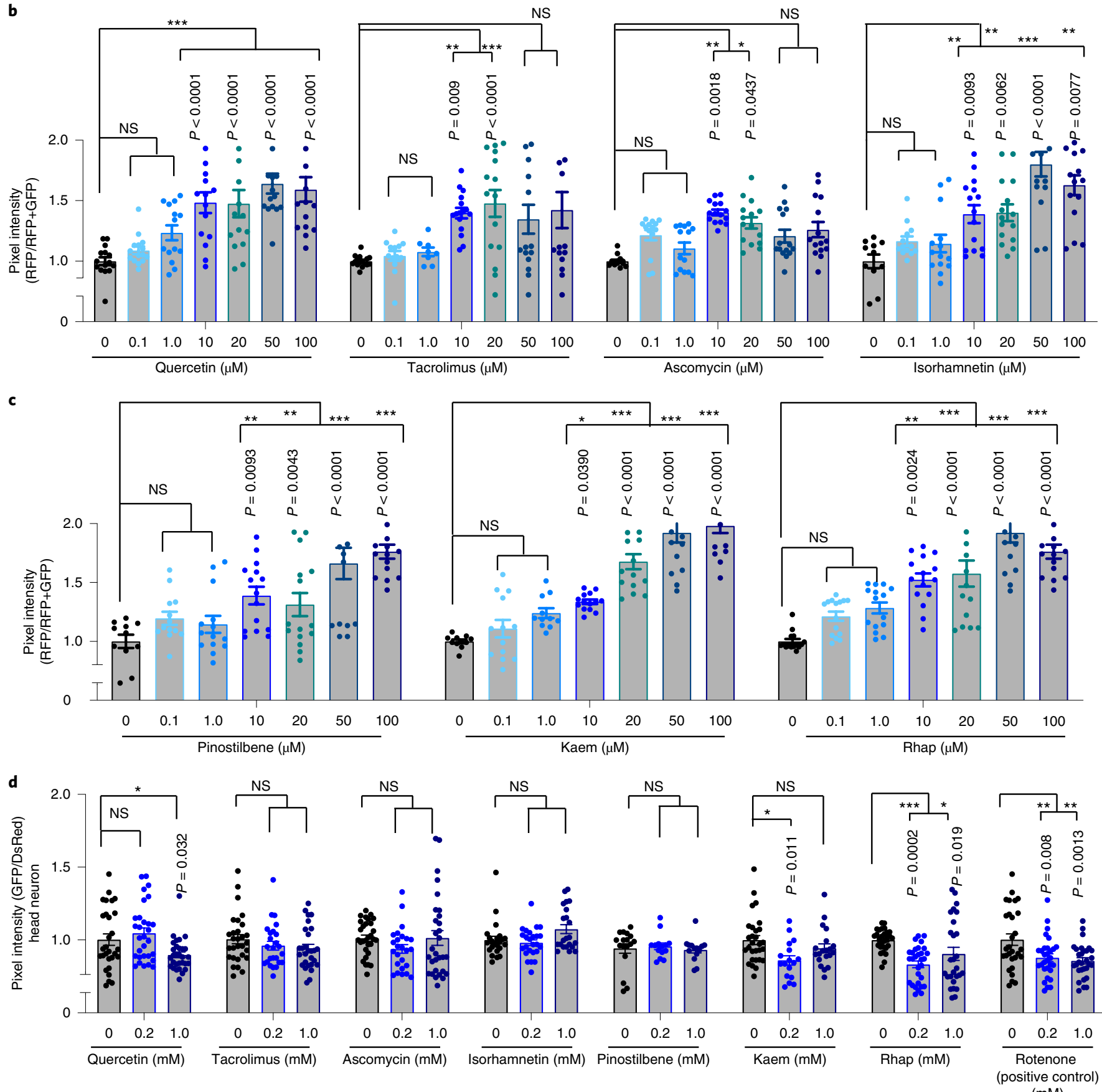
triggered upon Kaem and Rhap treatment, leading to the generation of smaller and fragmented organelles that can be easily engulfed by autophagosomes ${ }^{38}$. To further investigate whether Kaem- and Rhap-dependent memory improvement in the $\mathrm{hA} \beta_{1-42}$ worms is due to mitophagy induction (rather than any off-target effects), we introduced loss-of-function mutations (pink-1, pdr-1, dct-1) or knocked down selected genes (sqst-1, bec-1, via RNAi) on the basis of PCR and immunoblot data. Depletion of $p d r-1$ and $d c t-1$ abrogated Kaem-dependent memory improvement in the hA $\beta_{1-42}$ worms, whereas Rhap-induced memory improvement was dependent on the pink-1 pathway (Fig. 4c(left) and Supplementary Fig. 5f). Collectively, Kaem and Rhap appear to trigger mitophagy through the upregulation and/or activation of specific mitophagy components and via the modulation of mitochondrial dynamics.

To uncover the mechanisms of memory retention mediated by Kaem and Rhap at a cellular level, we asked whether these compounds act through the glutamatergic or cholinergic neurons, which are both impaired in $\mathrm{AD}^{39}$. Overexpression of $\mathrm{hA} \beta_{1-42}$ specifically in the glutamatergic neurons in worms $\left(\mathrm{hA} \beta_{1-42}\right.$ Glu $)$ has been shown to induce neurodegeneration ${ }^{36,40}$. We examined whether Kaem and Rhap could alleviate neurodegeneration and improve associative learning in these $\mathrm{hA} \beta_{1-42}{ }^{\text {Glu }}$ worms. Both Kaem and Rhap administered at $0.2 \mathrm{mM}$ improved memory deficits observed in $\mathrm{hA} \beta_{1-42}$ Glu; hApoE4 ${ }^{\text {Glu }}$ worms, as evidenced by the restoration of the chemotactic index to a level even surpassing that of hA $\beta_{1-42}$ Glu; hApoE$3^{\text {Glu }}$ control animals (Fig. 4c(right)). As previously described ${ }^{35,36}$, five specific glutamatergic neurons in the tail region of $C$. elegans hermaphrodites (LUA (R), LUA (L), PVR, PLM (R) and PLM (L)) (Supplementary Fig. $5 \mathrm{~g}($ left) and Video) afford a means of robust quantification of neurodegeneration in vivo. These anatomically isolated neurons facilitate unparalleled accuracy in scoring loss of neuronal processes and cell bodies at the single-neuron level, as they reproducibly degenerate in response to constitutive hA $\beta$ expression, and this occurs progressively with age ${ }^{40,41}$. Kaem inhibited glutamatergic neurodegeneration in adult Day $3 \mathrm{hA} \beta_{1-42}$ Glu; hApoE4 ${ }^{\text {Glu }}$ worms (Fig. $\left.4 \mathrm{~d}(\mathrm{left})\right)$. There was a trend towards glutamatergic neuroprotection by Rhap as a small percentage of worms reached 5 neurons, with fewer worms in the 1-2 neurons groups (Fig. $4 \mathrm{~d}(\mathrm{left}) ; P=0.0714)$. Indeed, in addition to inhibiting neuronal loss, administration of either Kaem or Rhap improved neuronal health as evidenced by increased GFP intensity (for example, in PVR neurons, Fig. 4d(right)) and also improved neuronal morphology (Supplementary Fig. 5g). Impairment of the cholinergic system plays an important role in the pathophysiology of $\mathrm{AD}$, and cholinergic therapies serve as a standard pharmacological approach in $\mathrm{AD}^{42}$. To investigate the effect of Kaem or Rhap on synaptic transmission of acetylcholine, pan-neuronal hA $\beta_{1-42}$ worms were exposed to an acetylcholinesterase inhibitor, aldicarb, and the time-course of paralyzing effects was scored ${ }^{43}$. While Kaem had no detectable effect on aldicarb-induced paralysis, Rhap greatly improved resistance of the $\mathrm{hA} \beta_{1-42}$ worms to aldicarb-induced paralysis, indicating possible cholinergic protection by Rhap (Fig. 4e and Supplementary Fig. 6a,b). The aldicarb-hypersensitive strain VC223 and the aldicarb-resistant NM204 were used as controls (Supplementary Fig. 6g).

We further asked whether Kaem and Rhap could reduce hA $\beta_{1-42}$ production by using N2a mouse neuroblastoma cells expressing the Swedish K595N and M596L mutations in Amyloid-beta precursor protein/APP (APPSwe). Both Kaem and Rhap reduced full-length APP, CTF- $\alpha$ (non-amyloidogenic pathway via $\alpha$-secretase) and CTF- $\beta$ (amyloidogenic pathway via $\beta$-secretase) in these mouse neuroblastoma cells in a dose-dependent manner, suggesting a possibility of reduction in amounts of CTF- $\beta$ available to be cleaved to $\mathrm{hA} \beta_{1-42}$ via $\gamma$-secretase (Extended Data Fig. $1 \mathrm{c}-\mathrm{f}$, and further verified in mouse brain detailed below). In summary, Kaem and Rhap improve the function or survival of glutamatergic and cholinergic neurons and reduce $\mathrm{hA} \beta_{1-42}$ production.

Kaem and Rhap improve memory by reducing Tau pathologies. In addition to alleviating $A \beta$ pathology, we asked whether Kaem and Rhap protect against tauopathies by using the well-characterized CK12 (hTau4R1N(P301L) ${ }^{44}$, pan-neuronal expression) and BR5270 (with pro-aggregant hTau(F3 280$)$ tau fragment ${ }^{45}$, pan-neuronal expression) strains. While the hTau(P301L) worms are known to have impaired memory ${ }^{11}$, both Kaem and Rhap improved memory in this Tau strain (Fig. 5a,b). Tacrolimus, Ascomycin, Isorhamnetin or Pinostilbene did not show associative memory improvement to the hTau $(\mathrm{P} 301 \mathrm{~L})$ worms at $1 \mathrm{mM}$, but Tacrolimus and Isorhamnetin improved associative memory at $0.2 \mathrm{mM}$ (Supplementary Fig. 5b-e).

Kaem increased mRNA levels of $d c t-1$, pink-1, pdr-1 and bec-1, while having no effect on sqst-1,vps-34 or skn-1 in the hTau(P301L) worms (Fig. $5 \mathrm{c}$ ). Rhap treatment resulted in increased mRNA levels of pink-1, sqst-1, vps-34 and skn-1 without having an effect on dct-1, pdr-1 or bec-1 (Fig. 5d). Kaem-induced memory improvement in hTau(P301L) worms was in a pink-1-, dct-1- and bec-1-dependent manner; Rhap-induced memory improvement in hTau(P301L) worms was in a dct-1-dependent manner (Fig. 5e). Furthermore, both Kaem and Rhap greatly improved the resistance of the hTau(P301L) worms against aldicarb-induced paralysis, indicating possible cholinergic protection (Supplementary Fig. 6c,d). Further investigation into the effects of Kaem and Rhap in hTau(F3 $\Delta 280)$ worms showed that both Kaem and Rhap improved the memory capacity in these worms $^{11}$ (Extended Data Fig. 3a). Mechanistically, although Kaem and Rhap had no or only minor changes in pink-1 and $p d r-1$ mRNA levels (Extended Data Fig. 3b), Kaem- and Rhap-induced memory improvement in the hTau(F3 $\Delta 280)$ worms was in a pink-1- and pdr-1-, but not dct-1-dependent manner (Extended Data Fig. 3c,d). Furthermore, both Kaem and Rhap greatly improved the resistance

Fig. 3 | Kaem and Rhap induce mitophagy in cells, C. elegans neurons and mouse brain. a, Effects of Kaem and Rhap on the protein levels of mitofusin2 (MFN2), YFP-Parkin and Tim23 in HeLa cells stably overexpressing YFP-Parkin. b-d, Semi-quantification of $\mathbf{a}(n=3$ biological replicates). e, Effects of Kaem and Rhap on the protein levels of MFN2, YFP-Parkin and Tim23 in HeLa cells stably overexpressing Mito-GFP and mCherry-Parkin. f-h, Semi-quantification of $\mathbf{e}(n=3$ biological replicates). For $\mathbf{a}$ and $\mathbf{e}$, CCCP was used as positive control. $\mathbf{i}$, Images showing co-localization of mitochondria (Mito-GFP) and lysosomes (LAMP1 antibody) under Kaem and Rhap (20 uM, 24 h) administration in GFP-mito-mCherry-Parkin HeLa cells. White arrows indicate mitophagy events. $\mathbf{j}$, Quantification of $\mathbf{i}$ with data from 3 biological repeats with around 5-7 images per biological repeat. $\mathbf{k}$, Transgenic nematodes were treated with Kaem and Rhap (both with $0.01,0.05,0.1$ and $0.2 \mathrm{mM}$ ), with mitophagy events calculated by the co-localization between the autophagic marker DsRed::LGG-1 and the mitophagy receptor DCT-1::GFP in neurons. $n=18-20$ neurons from 2 biological repeats. While the left panel shows one representative set of images, quantitative data are shown in the right panel. I, Effects of Kaem and Rhap on the induction of neuronal mitophagy in worms with $\mathrm{mt}$-Rosella reporter. Data were pooled from 2 biological replicates (total $n=20-35$ nematodes per group), with the results shown as mean \pm s.e.m. $\mathbf{m}, \mathbf{n}$, Data of quantified electron microscopic images showing effects of Kaem and Rhap on mitochondrial morphology and mitophagy-like events in mt-Keima HeLa cells $(\mathbf{m})(20 \mu \mathrm{M}$ for $24 \mathrm{~h})$ and mouse hippocampal brain tissues (n) (100 mg kg-1 $\mathrm{d}^{-1}$ via oral gavage from 12 months for 7 consecutive days; $n=3$ mice per group, with 4 random hippocampal neuronal images per mouse). Representative images are shown in Extended Data Fig. 1a,b. All quantitative data are shown as mean \pm s.e.m. One-way ANOVA followed by Šidák's multiple comparisons test; ${ }^{\star \star} P 0.01,{ }^{\star \star \star} P 0.001$. Original unprocessed western blot gel data are in Source Data Fig. 4. 
of the hTau(F3 $\Delta 280)$ worms against aldicarb-induced paralysis, indicating possible cholinergic protection (Supplementary Fig. $6 \mathrm{e}, \mathrm{f})$. In addition to memory benefit, Kaem and Rhap also improved healthspan measured as an enhancement of pharyngeal pumping in the hTau(P301L) worms (Extended Data Fig. 3f), although no statistical significance in improved mobility was observed (Extended Data Fig. 3g).
We then examined whether Kaem or Rhap supplementation exerts a beneficial effect by reducing pathological Tau aggregates. Phosphorylations of designated Tau sites are essential for Tau aggregation. Indeed, Kaem and Rhap diminished Tau phosphorylation levels at multiple sites such as Thr181, Ser202/Thr205 and Thr231 in mammalian HEK293-Tau P301L cells (Fig. 5f). Considering that Tau fibrils mediate transmission of neurofibrillary tangles a
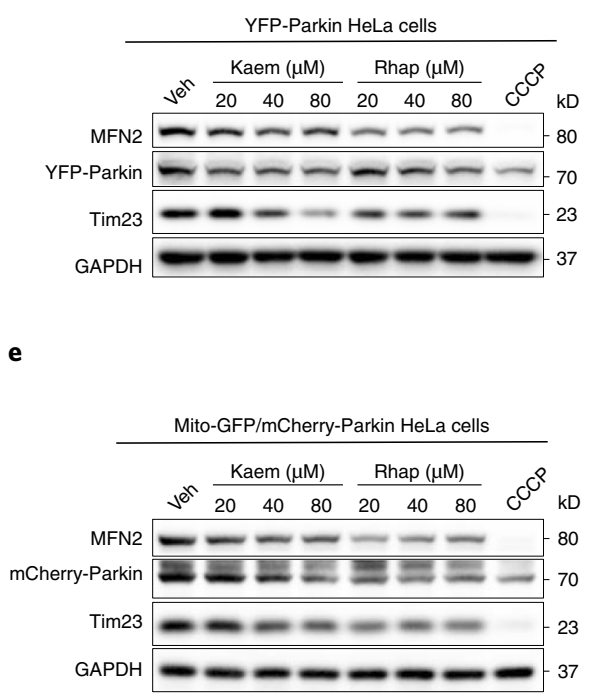

b

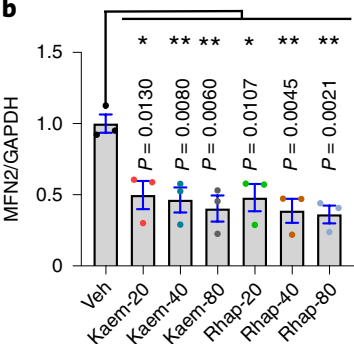

f

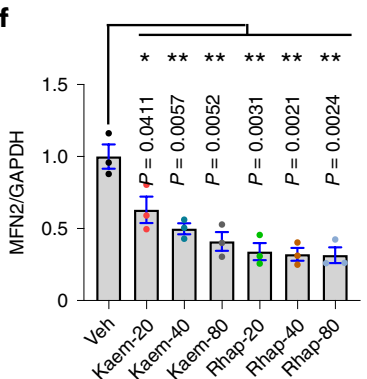

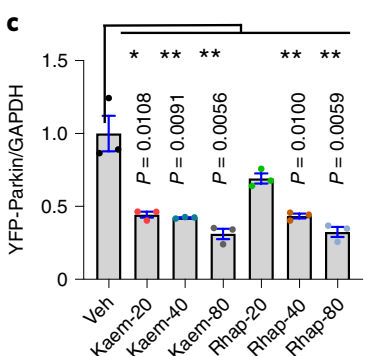

$\mathbf{g}$

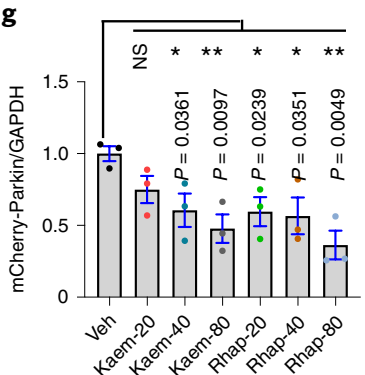

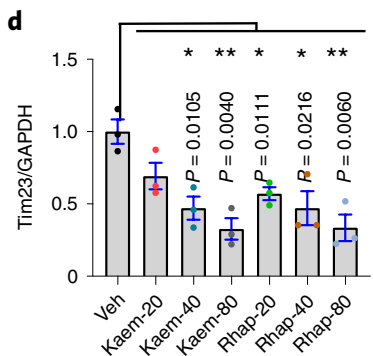

h

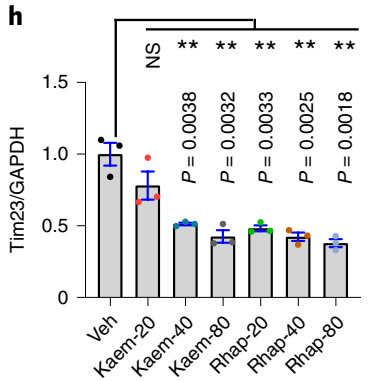

i
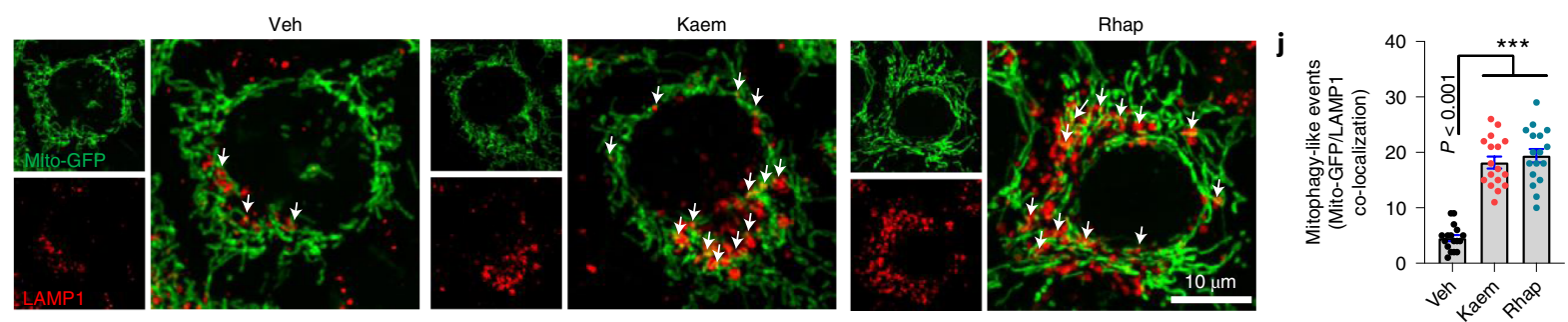

k
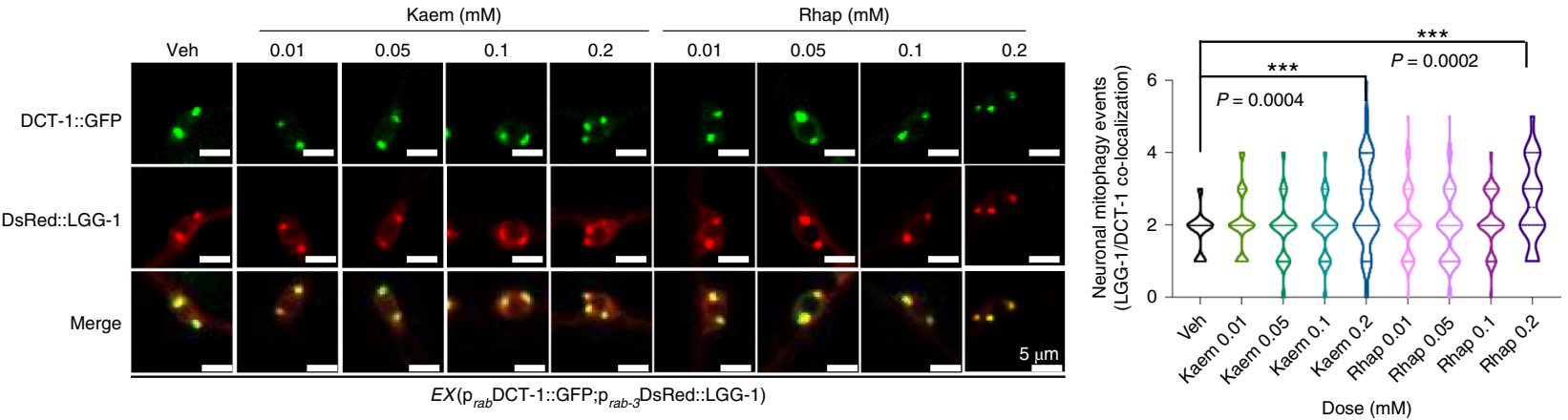

I

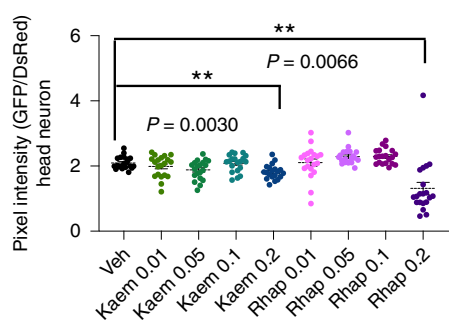

Dose (mM)

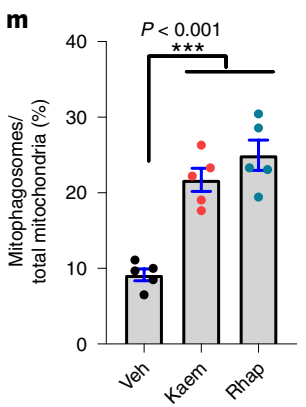


a

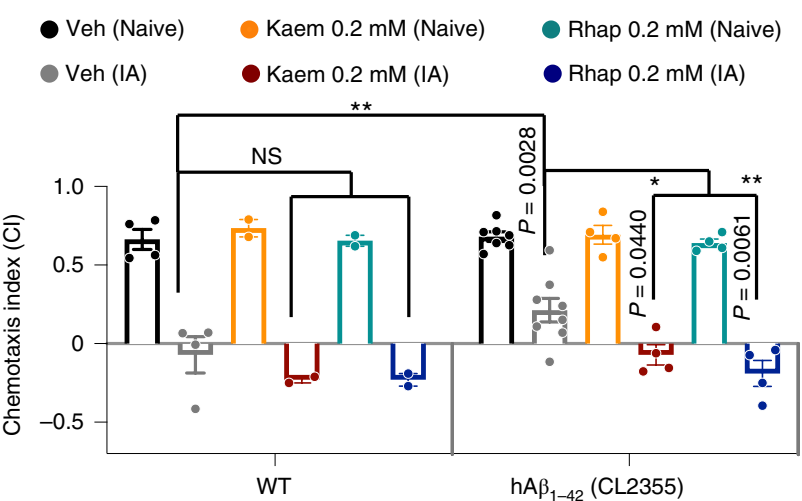

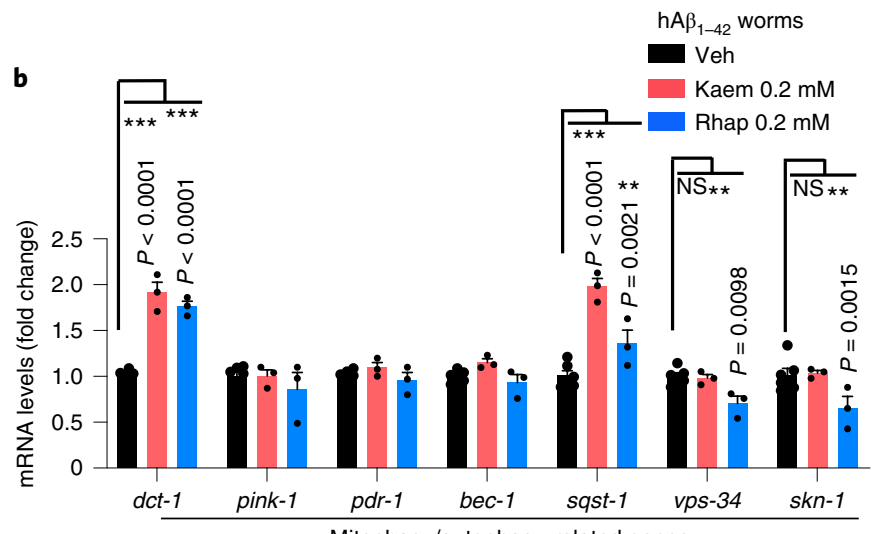

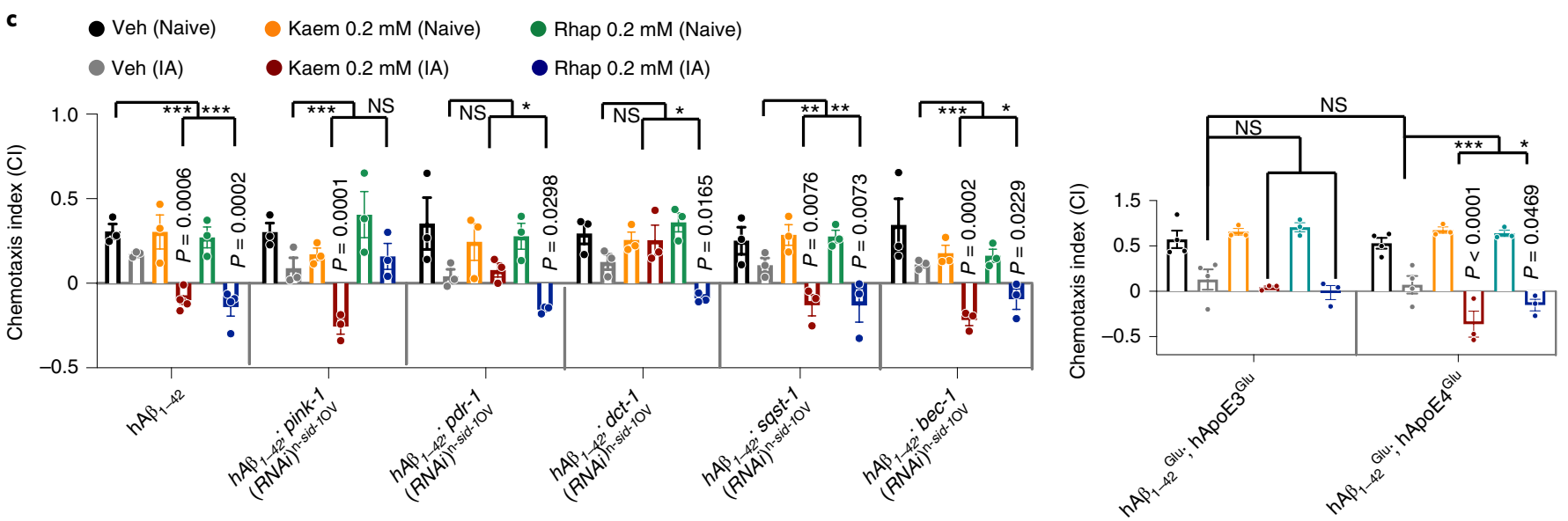

d
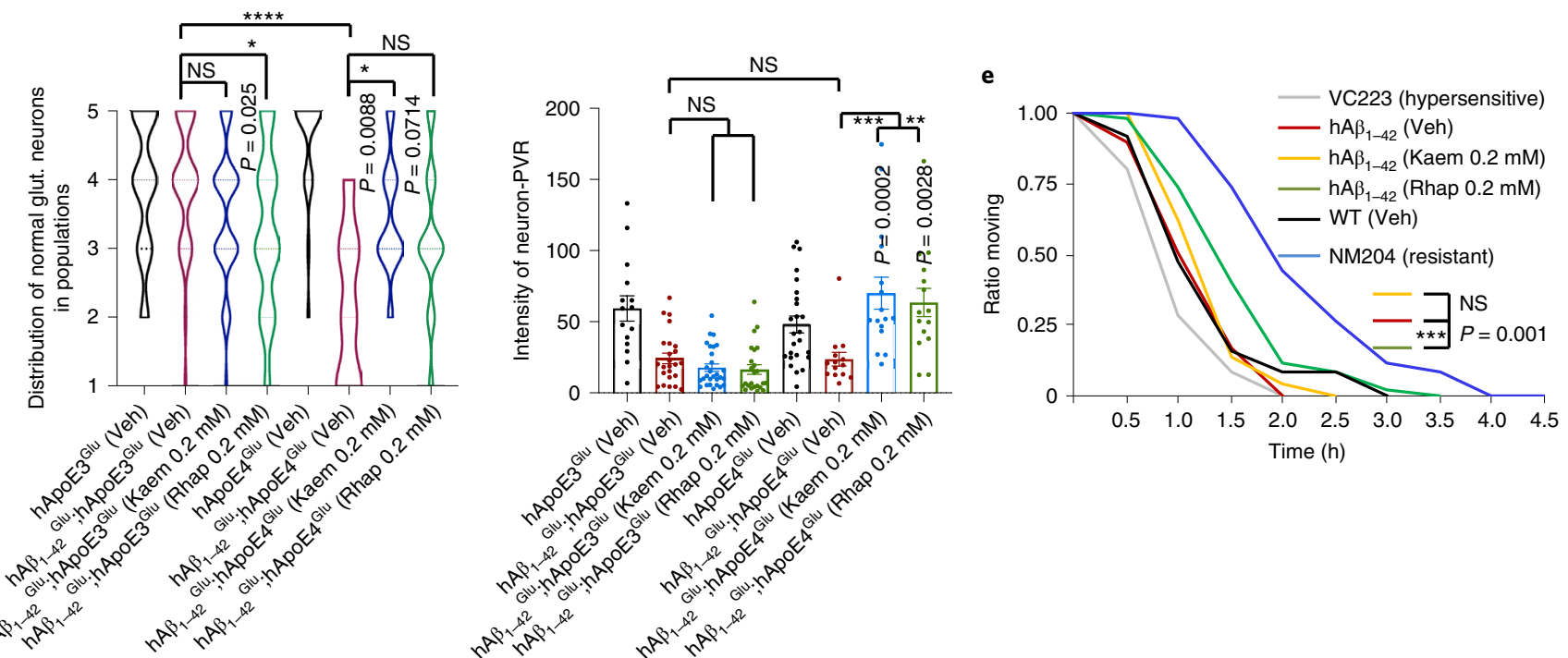

Fig. 4 | Mitophagy stimulation restores memory deficit and abrogates pathologies in AD-like A $\beta$ worms, and regulates cellular $A \beta$ production in mouse neuroblastoma cells. a, Effects of Kaem and Rhap on associative memory in adult day 1 WT and hA $\beta_{1-42}$ (CL2355) worms. Data were pooled from at least 4 biological replicates. $\mathbf{b}$, Effects of Kaem and Rhap on designated gene expression in day 1 adult worms. Data are from 1 representative biological repeat (3 technical repeats) from a total of 3 biological replicates. c, Left: effects of pink-1, pdr-1, dct-1, sqst-1 and bec-1 on Kaem- and Rhap-dependent memory improvement in the $\mathrm{hA} \beta_{1-42}$ (CL2355) worms. Right: effects of Kaem and Rhap on associative memory in adult day $1 \mathrm{hA} \beta_{1-42}$, , $\mathrm{hApoE3}{ }^{\mathrm{Glu}}$ (UA353) and hA $\beta_{1-}$ ${ }_{42}$ Glu.hApoE4 Glu (UA355) worms. 'Glu' denotes that either hA $\beta_{1-42}$ or hApoEs were expressed only in the glutamatergic neurons. Data were pooled from at least 4 biological replicates. d, Effect of Kaem or Rhap on glutamatergic neuroprotection in the hA $\beta_{1-42}$, Glu.ApoE4 worms and other worm strains. Left: distribution of worms with different numbers of 5 designated tail neurons ( $n=80-100$ from 2 biological replicates). Right: the fluorescent intensity of PVR neurons ( $n=15$ from 2 biological replicates). e, Effects of Kaem and Rhap on acetylcholinesterase inhibitor aldicarb-induced paralysis. VC223 (a strain hypersensitive to aldicarb-induced paralysis) and NM204 (a strain resistant to aldicarb-induced paralysis) were used as controls. All quantitative data are shown as mean \pm s.e.m. Two-way ANOVA followed by Tukey's multiple comparisons test (a-e); NS, no significance; ${ }^{\star} P<0.05$, ${ }^{\star \star} P<0.01,{ }^{\star \star \star} P<0.001$. Effects of Kaem and Rhap on $A \beta$ generation in mouse neuroblastoma cells are shown in Extended Data Fig. 1c-f. Additional data related to e are in Supplementary Fig. 6. 
(a possible hypothesis of age-dependent spreading of Tau pathology in $\mathrm{AD}$ patients $\mathrm{s}^{4-48}$ ), we asked whether Kaem and Rhap could alleviate 'seeded Tau'-induced Tau pathology. To explore this angle, we employed a high-content microscopy-based assay using HEK293 cells stably expressing the 0N4R isoform of human Tau, bearing the P301S mutation with a C-terminal Venus fluorescent protein $\operatorname{tag}^{49}$. It has been reported that ectopic addition of recombinant heparin-assembled P301S Tau (Tau seeds with no Venus tag) promoted the generation of intracellular bright Tau foci from a disperse distribution, indicating extracellular inclusion of pathological Tau seed-induced intracellular Tau aggregation ${ }^{49}$ (Extended Data Fig. 3h,i). While Rhap had no statistically significant effect on the reduction of Tau seed-induced intracellular Tau aggregation from a dose range between $3.125 \mu \mathrm{M}$ to $25 \mu \mathrm{M}$, Kaem dramatically reduced intracellular Tau aggregation in a dose-dependent manner, with $25 \mu \mathrm{M}$ showing over $80 \%$ reduction (Fig. $5 \mathrm{~g}$ ). Moreover, we assessed whether Kaem or Rhap affects the degradation of Tau aggregates; however, both did not reduce the numbers of Tau foci at up to $25 \mu \mathrm{M}$ for $24 \mathrm{~h}$ (Extended Data Fig. 3j,k). Kaem-induced decrease of seed-induced Tau aggregation was not due to cell death, as we did not detect any side effect on cell viability even up to $50 \mu \mathrm{M}$ of Kaem for $24 \mathrm{~h}$ (Extended Data Fig. 31). To conclude, Kaem and Rhap improve memory in Tau-expressing nematodes, as well as antagonize multiple p-Tau sites and/or Tau aggregation.

Effects of combined treatment and other factors on the hTau worms. We further investigated whether a combination of Kaem and Rhap had any additive or synergistic effect to combat memory loss in the $\mathrm{AD}$ worms. In the transgenic hTau $(\mathrm{P} 301 \mathrm{~L})$ worms, a dose of either $0.2 \mathrm{mM}$ Kaem or Rhap was able to improve memory. When administered together, we did not observe any additive or synergistic benefit in memory improvement (Fig. 5h). Further, since population studies indicate that individuals with $\mathrm{AD}$ have a shorter lifespan than healthy controls ${ }^{50,51}$, we examined whether enhanced memory (Fig. 5a,b) and healthspan (Extended Data Fig. 3f) also correlated with an increased lifespan. While the hTau(P301L) worms had a shorter lifespan compared with the WT N2 worms (Extended Data Fig. 1g), Kaem $(0.2 \mathrm{mM})$ or Rhap $(0.2 \mathrm{mM})$ administration extended the WT N2 nematode average lifespan by $24.5 \%$ and $32.5 \%$, and hTau(P301L) nematode lifespan by $5.8 \%$ and $17.4 \%$, respectively (Extended Data Fig. 1h,i and Supplementary Table 4). No synergistic effects in lifespan extension were noticed when combining Kaem $(0.2 \mathrm{mM})$ and Rhap $(0.2 \mathrm{mM})$ in either hTau(P301L) worms or N2 control animals (Extended Data Fig. 1h,i and Supplementary Table 4).

The changes in the biological effects (for example, chemotaxis-based memory performance) on the worms as induced by the tested compounds may also be attributable to alternate mechanisms, such as microbial metabolism (for example, dependence on the live food Escherichia coli (OP50) to metabolize the metabolic precursors to more bioactive ones) or changes in eating pattern due to a dietary restriction mimetic ${ }^{52,53}$. We performed additional analyses looking into the potential effect of microbial metabolism and eating habits on memory. Since both Kaem and Rhap were supplemented in the hTau(F3 $\Delta 280)$-expressing worms fed dead OP50, memory improvements were not likely to have been affected by microbial metabolism (Extended Data Fig. 3e). Likewise, exposing the worms to Kaem and Rhap likely did not reduce short-term food intake, but rather increased it; this may be due to the increased pharyngeal pumping observed with drug treatment (Extended Data Fig. 3f), thus excluding any indirect effects arising from dietary restriction.

Kaem and Rhap forestall pathologies in the 3xTg AD mice. Encouraged by the in silico, in vitro and nematode data, we wondered whether the anti-AD effects would translate to rodents. Therefore, we decided to test this using classical 3xTg AD mice bearing both $A \beta$ and Tau pathologies ${ }^{54}$. We treated the $3 \times \mathrm{Tg} \mathrm{AD}$ mice from 12.5 months with both compounds $\left(100 \mathrm{mg} \mathrm{kg}^{-1} \mathrm{~d}^{-1}\right)$ via oral gavage for 2 consecutive months and subsequently assessed memory and pathologies. In line with the results obtained using the C. elegans AD models, both Kaem and Rhap greatly improved spatial learning and memory in the Morris water maze test in terms of latency to the platform from days 1 to 6 (Fig. 6a,b), and in-platform frequency for the probe trial at day 7 (Fig. 6c). Similarly, Kaem and Rhap improved spatial memory in 3xTg AD mice tested using the $\mathrm{Y}$ maze spontaneous alternation performance test (Fig. 6d). Both molecules enhanced visual recognition memory when tested with a novel object recognition (NOR) test (Fig. 6e) compared with WT mice (Veh).

We further asked whether improved cognition in the $3 \mathrm{xTg}$ AD mice was due to the alleviation of $A \beta$ and Tau pathologies. Indeed, Kaem and Rhap comparably reduced insoluble $A \beta_{1-40}$ (Fig. 6f), as well as soluble and insoluble levels of $A \beta_{1-42}$ (Fig. $6 \mathrm{~g}$ ) in the hippocampal tissue of the $3 x T g \mathrm{AD}$ mice; consistently, an anti-A $\beta_{1-16}$ (clone $6 \mathrm{E} 10$ )-antibody probed immune-fluorescent evaluation showed reduced $A \beta$ load per region of interest (ROI) (Fig. 6h,i). Mechanistically, Kaem and Rhap reduced $A \beta$ production as supported by decreased levels of FL-APP, CTF- $\beta$ and CTF- $\alpha$ in the hippocampal tissue of the $3 \times$ Tg $\mathrm{AD}$ mice (Fig. $6 \mathrm{j}-1$ and Extended Data Fig. 4a), mirroring the results seen in the N2a mouse neuroblastoma cells (Extended Data Fig. $1 c-f)$. While microglia use energy (generated mainly by mitochondria) to clear $A \beta$ plaques, we recently reported over $60 \%$ reduction of microglial mitophagy in the hippocampal tissue from an APP/PS1 mouse model, which could be a cause for the reduced phagocytosis of $\mathrm{A} \beta$ plaques in $\mathrm{AD}$; mitophagy stimulation increased microglial phagocytosis in the APP/PS1 mice ${ }^{11,55}$. Accordingly, we evaluated whether Kaem and Rhap influenced microglial activity and whether the increased microglial activity could enhance the removal of $\mathrm{A} \beta$ plaques. Indeed, Kaem and Rhap increased phagocytic engulfment of $\mathrm{A} \beta$ plaques by microglia from $35.4 \%$ (microglia with $\mathrm{A} \beta /$ total microglia, Veh Group) to $83.0 \%$ and $72.6 \%$, respectively (Extended Data Fig. 4d,e). Kaem and Rhap also increased microglial populations in the $\mathrm{AD}$ hippocampus from 8.3 (number per region of interest) to 15.7 and 13.7, respectively (Extended Data Fig. 4d). Further, Kaemand Rhap-treated mice displayed a decreased number and length of microglial processes, highlighting their shift towards a phagocytic state (Extended Data Fig. 4d).

Fig. 5 | Mitophagy stimulation restores memory deficit in the AD-like $h$ Tau(P301L) C. elegans model and inhibits Tau pathologies in mammalian cells. $\mathbf{a}, \mathbf{b}$, Effects of Kaem (a) or Rhap (b) on associative memory in transgenic nematodes expressing hTau(P301L) (CK12). Data were pooled from 4 biological replicates. c,d, Effects of Kaem (c) and Rhap (d) on designated gene expressions in adult day 1 worms. Data are from a total of 3 biological replicates. e, Effects of pink-1, pdr-1, dct-1, sqst-1 and bec-1 on Kaem- and Rhap-dependent memory improvement in the hTau(P301L) worms. f, Western blot data with semi-quantifications showing changes in designated phosphorylated Tau sites in the HEK 293 cells expressing pTRE3G-mcherry-BI promoter-EGFP Tau P301L (HEK 293 3G-EGFP-Tau P301L/mCherry) with $24 \mathrm{~h}$ treatment of Kaem or Rhap. $\mathbf{g}$, Effects of Kaem and Rhap on seeded Tau-induced endogenous Tau aggregation in the HEK293 cells expressing ON4R P301S Tau-Venus. Data are from 3 biological replicates. h, Evaluation of any synergistic effects of Kaem and Rhap on associative memory in hTau(P301L) (CK12) worms. Data were pooled from 3 biological replicates. All quantitative data are shown as mean \pm s.e.m. Two-way ANOVA followed by Tukey's multiple comparisons test (a-h). NS, no significance; ${ }^{\star} P<0.05,{ }^{\star \star} P<0.01,{ }^{\star \star \star} P<0.001$. Additional Tau seeding data are included in Extended Data Fig. 3h-k. Original western blot gels for $\mathbf{f}$ are included in Source Data Fig. 2. 
We then examined aggregation levels of Tau in response to Kaem and Rhap treatment. While Kaem reduced $62.2 \%$ of AT8 (for p-Tau Ser202/Thr205)-positive cells, Rhap abrogated 73.5\% of AT8-positive cells in the hippocampus of the 3xTg AD mice (Fig. $6 \mathrm{~m}, \mathrm{n}$ ). At the molecular level, Kaem and Rhap dramatically reduced the phosphorylation levels of multiple classical p-Tau sites, a
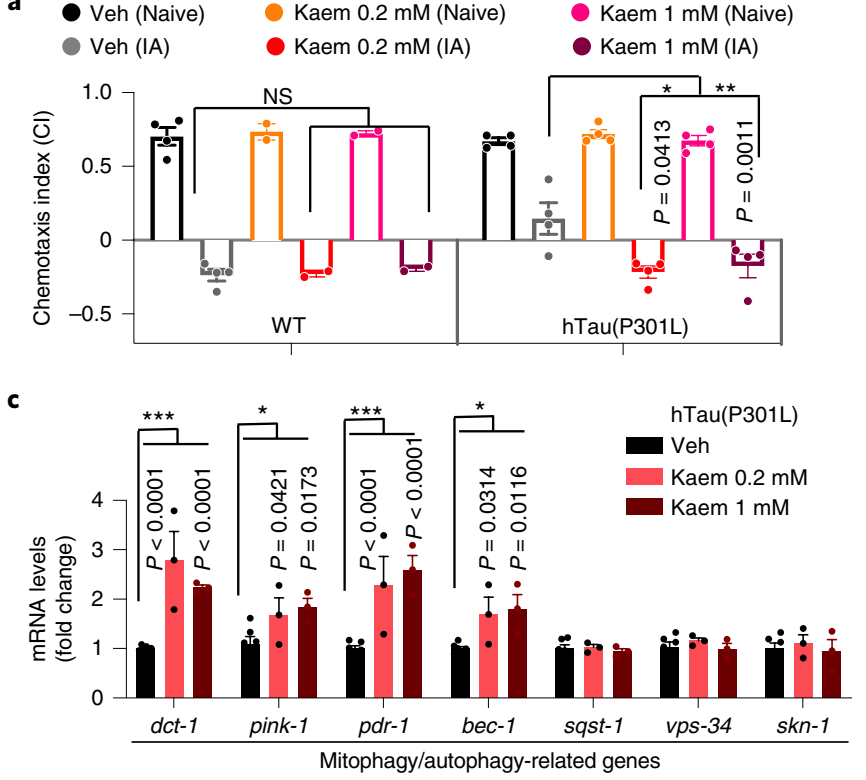

b Veh (Naive) Rhap $0.2 \mathrm{mM}$ (Naive) Rhap $1 \mathrm{mM}$ (Naive) Veh (IA) Rhap $0.2 \mathrm{mM}$ (IA) Rhap $1 \mathrm{mM}(\mathrm{IA})$
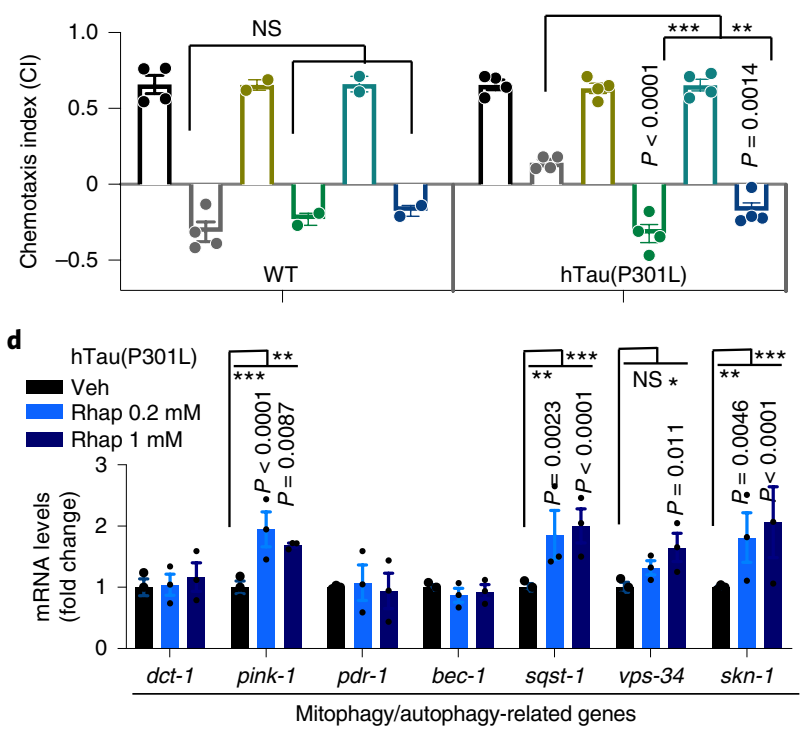
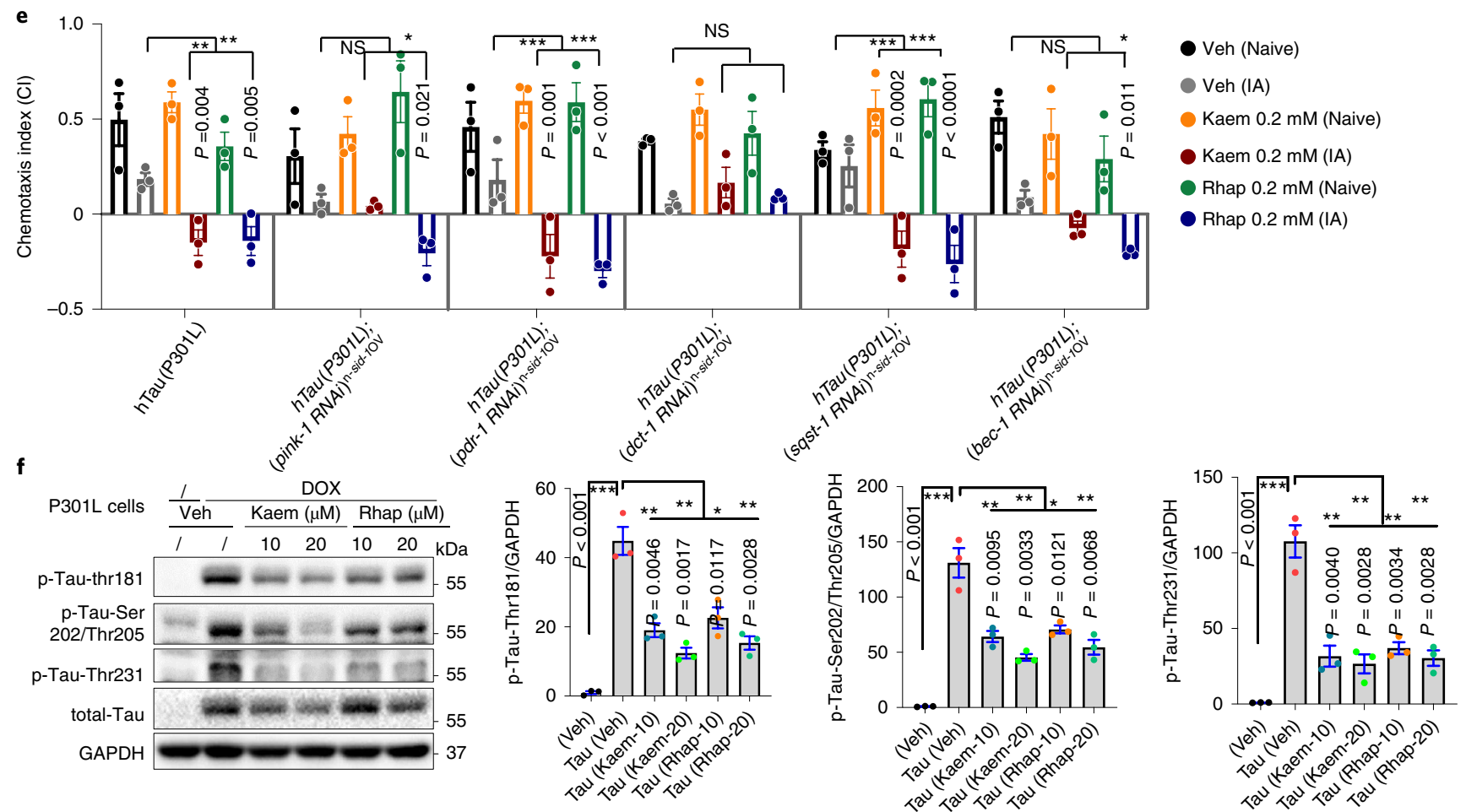

g Tau-seed (10 nM)

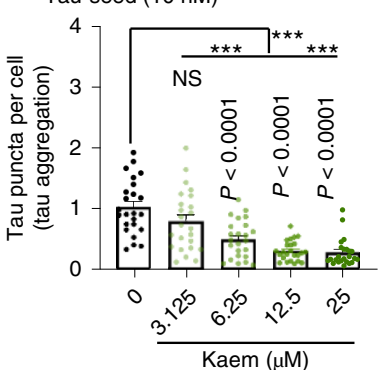

h

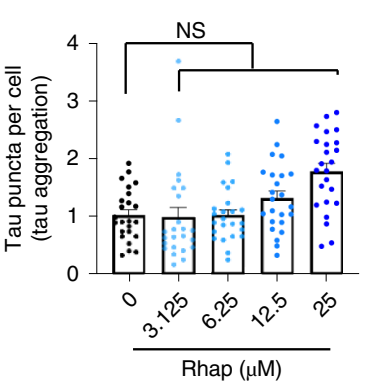

h

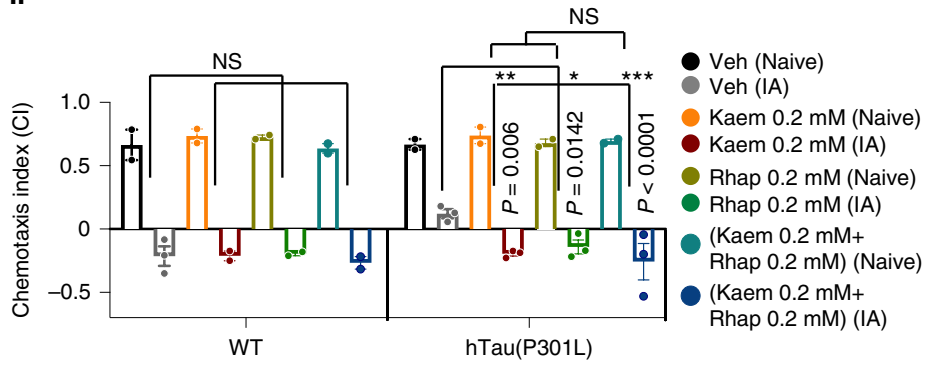




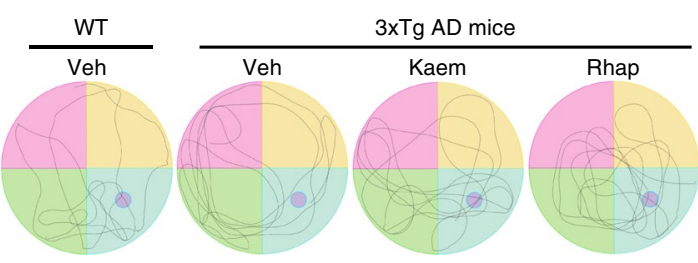

b

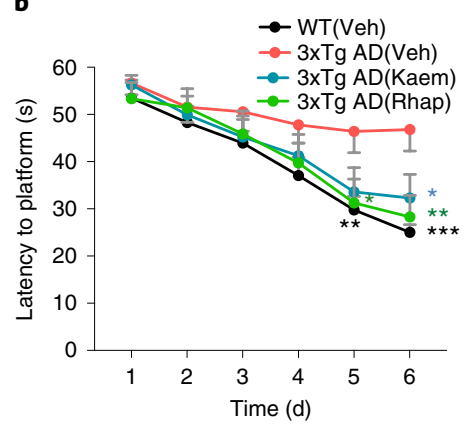

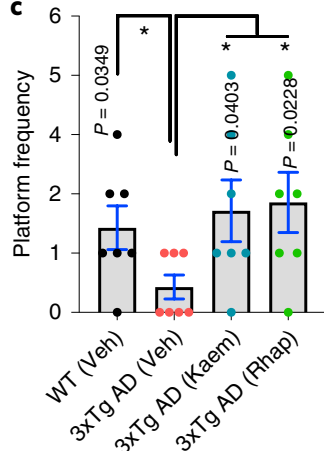
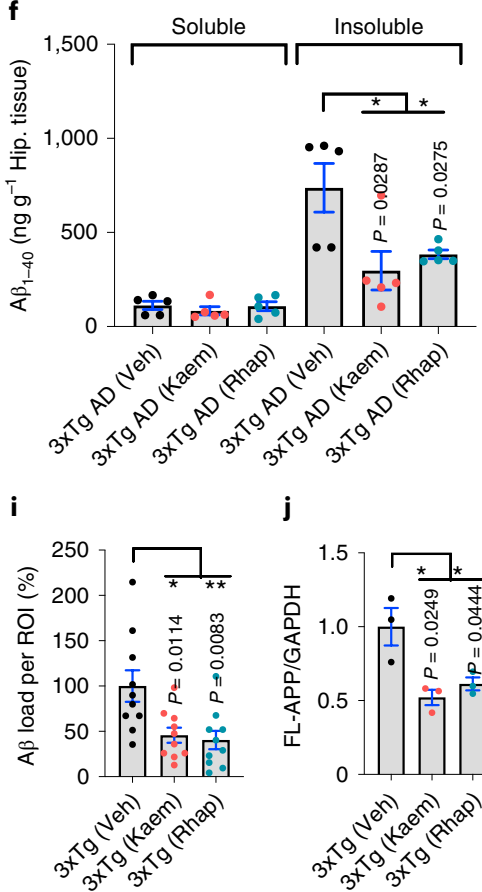

j

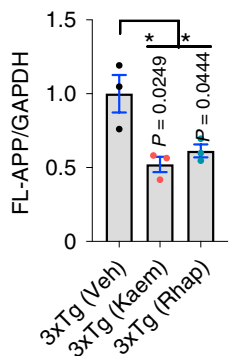

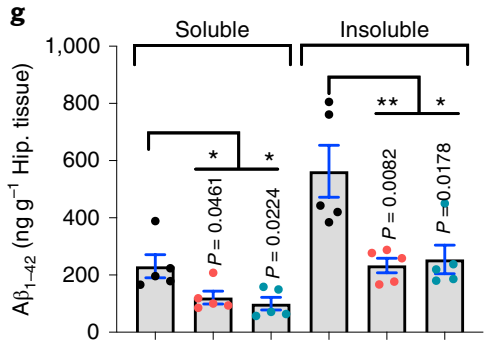

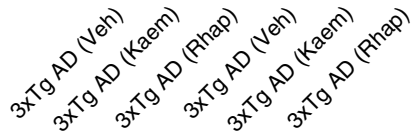

$\mathbf{k}$

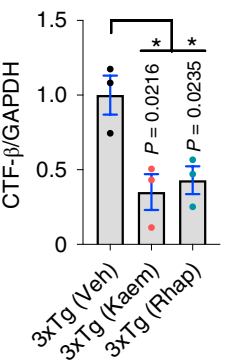

I

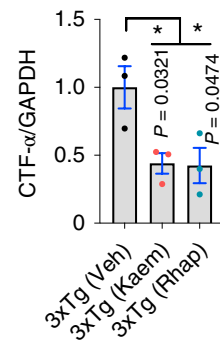

$\mathbf{0}$

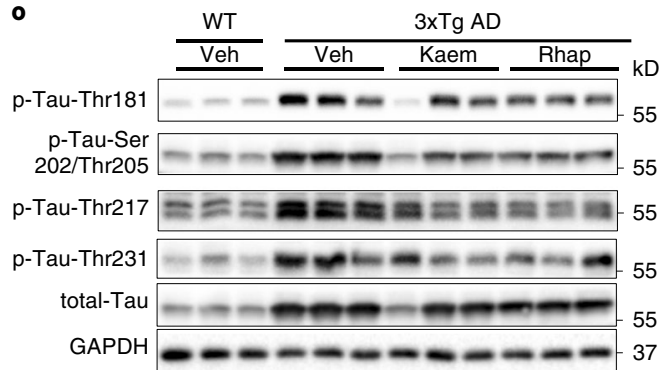

Fig. 6 | Mitophagy stimulation forestalls memory loss and ameliorates both $\mathbf{A} \boldsymbol{\beta}$ and Tau pathologies in $3 \times \operatorname{Tg}$ AD mice. The $3 \times \operatorname{Tg} A D$ mice were treated with Kaem or Rhap $\left(100 \mathrm{mg} \mathrm{kg}^{-1} \mathrm{~d}^{-1}\right)$ via oral gavage for 2 months starting from 12.5 months of age. a, Representative images of the swimming tracks of mice at day 7 in the Morris water maze test ( $n=6$ mice per group). $\mathbf{b}$, Latency to the platform of mice from days 1 to 6 . c, Platform frequency of mice in the probe trial at day 7. d,e, Effects of Kaem and Rhap on spontaneous alternation (d) (Y maze) and novel object recognition/NOR (e). $\mathbf{f}, \mathbf{g}$, Soluble and insoluble $A \beta_{1-40}$ and $A \beta_{1-42}$ levels in hippocampal tissues. $n=5$ mice in all groups. $\mathbf{h}$, Immunohistochemical analysis of $A \beta$ load in $3 \times T g$ AD mice hippocampi and cortices under Kaem or Rhap treatment. Experiments were repeated twice independently, with similar results. i, Quantification of A $\beta$ load per ROI in images from $\mathbf{h} . n=10$ random areas in the ROIs from 3 mice per group. j-I, Semi-quantification of western blot data showing effects of Kaem and Rhap on the levels of full-length APP (FL-APP), CTF- $\beta$ and CTF- $\alpha$ in hippocampal tissues from the $3 \times \operatorname{Tg}$ AD mice $(n=3$ biologically independent samples). $\mathbf{m}$, Representative immunofluorescence staining of AT8-positive cells in the cortex and hippocampus of 3xTg AD mouse brains. Experiments were repeated twice independently, with similar results. The blue and red squares denote designated brain regions were magnified. $\mathbf{n}$, Quantified data of $\mathbf{m}(n=10$ random areas in the ROls from 3 mice in each group). o, Effects of Kaem and Rhap on the expression levels of different p-Tau sites (Thr181, Ser202/Thr205, Thr217 and Thr231) in hippocampal tissues from the $3 \times \operatorname{Tg}$ AD mice ( $n=3$ mice per group). All quantitative data are shown as mean $\pm s$.e.m. Two-way ANOVA followed by Tukey's multiple comparisons test $(\mathbf{b}-\mathbf{g}, \mathbf{i}-\mathbf{I}, \mathbf{n})$. NS, no significance; ${ }^{\star} P<0.05,{ }^{\star \star} P<0.01,{ }^{\star \star \star} P<0.001$. Additional data on the mechanisms of mitophagy induction by Kaem and Rhap in mice are shown in Extended Data Fig. 4. Original western blot gels for $\mathbf{0}$ are included in Source Data Fig. 3. 
such as Thr181, Ser202/Thr205 and Thr231, by over 50\% (Fig. 60 and Extended Data Figs. 1j,k and 4b,c). Recent clinical studies point to a new p-Tau site, p-Tau217, which increases during early $\mathrm{AD}$ and correlates with $\mathrm{AD}$ with high sensitivity and discriminative accuracy ${ }^{56,57}$. To this end, we evaluated the effect of Kaem and Rhap on p-Tau217. Intriguingly, Kaem and Rhap caused a $57.8 \%$ and a $66.6 \%$ reduction in p-Tau217 expression, respectively (Fig. 60 and Extended Data Fig. 11). To investigate whether the Kaem- and Rhap-induced reduction in p-Tau was dependent on mitophagy, we knocked down PINK1 gene in HEK 293 (with Tau P301L) cells. Immunoblotting data show that PINK1 knockdown almost completely annulled the effect of Kaem and Rhap on the phosphorylation status of Tau, suggesting that these mitophagy inducers inhibit p-Tau (thr181) and p-Tau (thr217) via a PINK1-dependent pathway (Extended Data Fig. 1m-o).

We further checked mitophagy status in the $3 \times \mathrm{Tg} \mathrm{AD}$ mice. Immunoblotting data from the hippocampal tissue show that major mitophagy proteins, including PINK1, Parkin, OPTN and p-ULK1 (Ser555), were reduced in the 3xTg AD mice when compared with WT mice. Meanwhile, levels of these proteins were higher in Kaemand Rhap-fed AD mice (Extended Data Fig. 4f-j). In line with our results from cells and worms, our mouse data suggest that Kaem and Rhap restore impaired mitophagy in the $3 \times \mathrm{Tg} \mathrm{AD}$ mice. In line with the immunoblot data, electron microscopic analysis shows accumulated mitochondria in the hippocampal tissue of the $3 \mathrm{xTg} \mathrm{AD}$ mice, while this phenomenon was alleviated with Kaem or Rhap treatment accompanying increased mitophagy (Fig. $3 \mathrm{n}$ and Extended Data Fig. 1b). Furthermore, Kaem and Rhap increased protein expression levels of members of the mitochondrial OXPHOS complex (CV-ATP5A, CIII-UQCRC2 and CI-NDUFB8) in hippocampal tissue of 3xTg AD mice (Extended Data Fig. 4m).

While basal autophagy was lower in the hippocampal tissue of the 3xTg AD mice (lower LC3B-II/LC3B-I and higher p62) compared with WT mice, administration of Kaem and Rhap increased autophagy in the 3xTg AD mice (Extended Data Fig. 4f,k,l). Indeed, Kaem and Rhap promoted autophagy in vitro at $10 \mu \mathrm{M}$ (Extended Data Fig. $4 n-p$ ), a dose that also triggers mitophagy in vitro (Fig. 2c). However, Kaem- and Rhap-induced memory improvement in worms is dependent on key mitophagy genes, such as pink-1, pdr1 or $d c t-1 /$ NIX (Figs. 4c(left) and 5e, and Extended Data Fig. 3c). In addition, Kaem- or Rhap-induced inhibition of multiple p-Tau sites (Thr181 and Thr217) was dependent on the mitophagy gene PINK-1 (Extended Data Fig. 1m-o). Given the intertwined linkage between mitophagy and autophagy, we do not exclude the possibility of a Kaem- and Rhap-induced non-mitophagy-related autophagy in the contribution to the multiple behavioural and pathological benefits reported here. Collectively, these combined behavioural, histological and molecular data indicate the bioavailability and strong capacity of Kaem and Rhap to retard memory loss, while simultaneously decreasing both $\mathrm{A} \beta$ and Tau pathologies in the $3 \mathrm{xTg}$ AD mouse model.

\section{Discussion}

Aiming to unveil additional AD aetiologies and support the development of novel anti-AD drug candidates, we have established an in silico-in vitro-worm-mouse screening technology and validation approach to identify novel mitophagy modulators. Our method revealed two bioavailable neuronal mitophagy inducers with anti-AD potential, as they showed conserved memory retention capacity in one $A \beta$ nematode model, two Tau nematode models and the $3 \times \mathrm{Tg}$ AD rodent model. Compared with most clinical-trial-stage anti-AD drug candidates, which target only $\mathrm{A} \beta$ or Tau pathology ${ }^{7}$, these mitophagy inducers work on both. Mechanistically, they inhibit $A \beta$ pathology via production inhibition and microglial phagocytic clearance, abrogate Tau pathology via inhibition of multiple p-Tau sites (including p-Tau181, Ser202/Thr205, Thr231 and
Thr217) and reduce spreading of pathological Tau. Kaem and Rhap are likely biological safe as the doses used for C. elegans and mice did not show detectable side effects (Extended Data Fig. 5 and Fig. 6). E.g., based on the toxicity assay results, treatments with Kaem or Rhap at concentrations ranging from 0.01 to $1 \mathrm{mM}$ did not influence worm development (Extended Data Fig. 5a-d).

Over the past years, the scope of AI has moved from sheer theoretical knowledge to real-world applications, including drug development for aging and various diseases ${ }^{17,19,22,58}$. Our AI-driven virtual screening recommendation has achieved a $44 \%(8 / 18)$ success rate, which is much higher than traditional screening methods for small compounds. For instance, typical rates from experimental high-throughput screening have ranged between $0.01 \%$ to $0.5 \%$, while typical hit rates for prospective virtual screening have been reported at $1 \%-10 \%{ }^{59,60}$. Due to the low numbers of reference compounds, that is, mitophagy inducers (Supplementary Table 1), we have not been able to identify candidate compounds using machine learning approaches that may require large numbers of reference compounds (typically requiring between 100 to over 1,000 compounds) ${ }^{19,22}$. To overcome the shortage of reference compounds, we have chosen to use a combinational approach, combining simplified molecular-input line-entry system (SMILES) sequences, molecular interaction features and 3D conformers fingerprint, which can represent the molecule as a fixed bit-string on the basis of expert-encoded substructures, but do not require big training datasets $^{23}$. However, since the fingerprint techniques rely heavily on the manually designed functional groups in the local chemical environment, they cannot capture global structural and pharmacophore information, and thus usually underperform in the context of complicated biological systems ${ }^{61}$. More recently, several fingerprint-inspired unsupervised machine learning approaches (for example, Mol2 $\mathrm{vec}^{26}$ and $\mathrm{N}$-gram ${ }^{26}$ ) have rapidly advanced to utilizing larger compound datasets such as ChEMBL and ZINC for the comprehensive representation of molecules ${ }^{24,25}$, yielding gains in property prediction performance over manually crafted fingerprints and preliminary machine learning methods. Compared to the sparse fingerprint vectors, our method has provided an efficient and information-enriched molecular embedding technique via learning the global relationship and 'semantic similarity' of each component. This has encouraged us to bypass the large dataset required by an unsupervised machine learning regime and has established a pipeline for virtual screening in complicated biological systems on the basis of only a few data points. Our machine learning approach could be applied to the screening of additional new mitophagy stimulators in larger drug libraries. Advanced AI technologies, such as a trained deep neural network ${ }^{62}$, could be used to predict mitophagy induction activity in molecules that are structurally different from known mitophagy inducers in the future, when larger numbers of reference compounds become available.

Our findings reveal a conserved mechanism for memory loss that is at least partially mediated by defective mitophagy, while our in silico, high-accuracy screening technology paves the way for identifying exploitable mitophagy stimulators to improve healthspan and cognition in humans. Since mitochondria are essential for neuronal plasticity and survival, we have postulated that defective mitophagy driven by genetics, aging and/or other environmental factors, displays a causative role ahead of $A \beta$ and Tau pathologies in $\mathrm{AD}$ development and progression ${ }^{1,2,11}$. The current study further consolidates this hypothesis since key mitophagy pathways, including the PINK1/Parkin pathway, possess a central role in memory improvement upon supplementation with mitophagy-inducing compounds. Kaem is a natural flavanol available in a variety of plants including beans, tea, kale and broccoli ${ }^{63}$. Rhap is a stilbenoid/ phytoalexin, an antimicrobial compound produced by plants, such as Vitis species (grapes), etc. A well-known natural analogue of Rhap is resveratrol, which shows anti-aging and neuroprotective activi- 
ties in cross-species animal studies and clinical trials ${ }^{64,65}$. Preclinical studies have shown that the anti-AD potential of Kaem may lie in its anti-oxidative and anti-inflammatory activities ${ }^{66,67}$. Indeed, a recent ongoing community-based prospective cohort study with a total of 921 participants, suggests that taking flavonols, including Kaem, reduces the risk of $\mathrm{AD}^{63}$. The current study verifies the anti-AD potential of Kaem using both nematode and rodent models of $\mathrm{AD}$, and shows that mitophagy induction is a major molecular mechanism of neuroprotection for AD. We also identified Rhap as a robust mitophagy inducer able to potentially inhibit $A \beta$ and Tau pathologies in both worm and mouse models of $\mathrm{AD}$, in a largely mitophagy-dependent manner. The pharmacokinetic data for both Kaem and Rhap are available. Kaem can cross the blood-brain barrier, ${ }^{68-70}$ whether Rhap is able to cross the blood-brain barrier has yet to be determined. Future clinical studies should address the appropriate dose range, delivery routes and any potential side effects. To note, our mitophagy-targeting drug screening strategy also reduces the risk of eliminating potential anti-AD drug candidates that may have little or no capacity to induce mitophagy. Among the 18 AI-selected molecules, Tacrolimus was able to induce mitophagy in HeLa cells but not in worm neurons. However, Tacrolimus was able to improve memory in the hTau $(\mathrm{P} 301 \mathrm{~L})$ C. elegans model at a concentration of $0.2 \mathrm{mM}$ (but not at $1 \mathrm{mM}$ ) (Supplementary Fig. 5b). Tacrolimus, a calcineurin inhibitor and an immunosuppressant, inhibited $\mathrm{A} \beta$-induced dendritic spine $\operatorname{loss}^{71}$ and is scheduled for a pilot open-labelled clinical trial (NCT04263519).

Efficacy and safety determine clinical success in drug development ${ }^{72}$. A challenge in virtual screening and computer-aided drug design is the existence of 'pan-assay interference compounds' (PAINS), which are chemical compounds that normally cause false-positive results in high-throughput screens ${ }^{73-76}$. The application of carefully selected PAINS-related filters into machine learning/AI-based drug screening ${ }^{75}$, including the one we generated here, should be used. While we do not exclude the possibility that Rhap and Kaem could be hydroxylated, our strategy involving sophisticated in vitro analyses and multiple bioassays across two distinct in vivo experimental systems consistently shows the specificity and efficacy of the two lead compounds. Furthermore, the value of the effective dose of a drug candidate is a pivotal indicator of the potential of clinical studies. While the in vitro cell culture-based effective doses for many of the promising drug candidates are in the $\mathrm{nM}$ to $10 \mu \mathrm{M}$ range $\mathrm{e}^{7,77}$, there are drug candidates with effective doses in the $\mathrm{mM}$ range. For example, while nicotinamide adenine dinucleotide (oxidized form, $\mathrm{NAD}^{+}$) is reduced in aging and $\mathrm{AD}$, there are many clinical trials on the application of $\mathrm{NAD}^{+}$precursors, such as nicotinamide riboside and nicotinamide mononucleotide, in treating neurodegenerative diseases and multiple age-related diseases ${ }^{5}$. The effective doses for nicotinamide riboside/nicotinamide mononucleotide in cell culture are between 0.5 to $3.0 \mathrm{mM}$; the doses used in clinical trials range from 250 to $2,000 \mathrm{mg} \mathrm{d}^{-1}$ without detectable toxicity ${ }^{5,78}$. For Kaem and Rhap, no detectable toxicity was observed at $50 \mu \mathrm{M}$ in cells (effective dose $=10 \mu \mathrm{M})($ Extended Data Fig. 3l), at $0.5 \mathrm{mM}$ in C. elegans (effective dose $=0.2 \mathrm{mM}$ ) (Extended Data Fig. $5 \mathrm{a}-\mathrm{d})$ or at $\left(100 \mathrm{mg} \mathrm{kg}^{-1} \mathrm{~d}^{-1}\right)$ in mice (Fig. 6). These data strongly indicate that Kaem and Rhap are effective and safe in both cells and animals.

It is intriguing that aging and defective mitophagy converge with $\mathrm{AD}$-defining $\mathrm{A} \beta$ and Tau pathologies to inflict synaptic loss and neuronal death leading to subsequent cognitive dysfunction and brain homeostatic collapse. Although hyperactivation of mitophagy may not be without detrimental consequences for health, targeted mitophagic restoration is able to alleviate memory loss in a diverse set of nematode and rodent models of $\mathrm{AD}^{11,15,79}$. The broader impacts of mitophagy in targeting multiple aspects of AD pathogenesis, such as impaired proteostasis, inflammation, neuronal death, glial dysfunction and energy metabolism ${ }^{1,11,80}$, support the potential value of examining mitophagy inducers for AD mitigation in a clinical setting.

\section{Methods}

Machine learning-based screening of mitophagy inducers. Multi-representation models (1D, 2D and 3D) were first pre-trained to generate representations for compounds. The goal of the pre-training process is to generate comprehensive molecular representations for different molecules. The pre-training models can generate vector representations for molecules that are not included in the initial pre-training dataset. The compounds from the ChEMBL and ZINC database were filtered using the parameters noted in Jaeger $\mathrm{et}^{\mathrm{al}^{26}}$. We transformed the compounds into SMILES representations using $\mathrm{RDKit}^{81}$, which simplified multi-dimensional molecular structures into ordered text chains. In total, 19.9 million molecules were included in all further processes (hereafter called the pre-training dataset). A Mol2vec model was pre-trained on the basis of the pre-training dataset and new molecules could be described by summing the substructure vectors retrieved from a pre-trained Mol2vec model. The 2D pharmacophore fingerprint was implemented to introduce the fragment graph relationship between different molecules. Ehrlich ${ }^{82}$ defined a pharmacophore as "a molecular framework that carries (phoros) the essential features responsible for the biological activity of a drug (pharmakon)". Therefore, the structural similarity between different molecules could be captured on the derived distance between the topological distributions of the various atoms that were inherent in the function of the molecules themselves. The $2 \mathrm{D}$ pharmacophore fingerprint was obtained using $\mathrm{RDKit}^{81}$, producing a vectorized prioritization of molecules based on the $2 \mathrm{D}$ topological similarity (that is, the fingerprint). The $3 \mathrm{D}$ conformers fingerprint method, initially described by Landrum et al., was introduced to augment the 3D information ${ }^{83}$. The original dataset (with an initial 19.9 million compounds) was again run through RDKit using the $3 \mathrm{D}$ conformer fingerprint module. This technique used information about the features (chemically active areas) on a molecule, producing a 'feature-map vector' that captured information around the projected biological activity of molecules.

We applied the 1D, 2D and 3D fingerprints to identify potential mitophagy inducers within the screening process. The similarity score for each of the 3,724 compounds with the filtered molecules was calculated, with a higher score indicating a higher degree of similarity. We defined the strength of the relationship from the three models trained by different information, that is, 1D-molecules semantic information, 2D-fragments graph relationship and 3D-atomic bonds, spatial angle and length as the similarity. The similarity score was calculated as:

$$
s=v * w,
$$

where $s$ is the similarity score, ${ }^{*}$ is the vector dot product, and $v$ (the inductor vector) and $w$ (the compound vector) are high-dimensional vector representations from the pre-trained model.

We clustered the representation based on the 14 mitophagy inducers scored by the similarity function (equation 1). We then filtered the outliers and ranked the low-score compounds on the basis of filter function (equation 2).

$$
\text { filter function }=\left\{\begin{array}{cc}
0 & s=1 \\
s & \theta \leq s<1 \\
0 & s<\theta
\end{array},\right.
$$

where $s$ is the similarity score and $\theta$ stands for the cut-off value $(=0.75)$. The molecules with a similarity ranking score $\geq 0.75$ were selected. In total, the selection method described here produced 18 novel analogue compounds for validation (Supplementary Table 2).

Small compounds in the Macau Library. All AI-selected compounds were from the State Key Laboratory of Quality Research in Chinese Medicine, Institute of Chinese Medical Sciences, University of Macau, Macau SAR, China (J.-H.L.). All molecules were of at least $98 \%$ purity and dissolved in dimethylsulfoxide (DMSO) for experiments. For the in vitro and in vivo experiments, Kaem (HY-14590) and Rhap (HY-N2229) were purchased from MedChemExpress, with $99.62 \%$ and $99.66 \%$ purity, respectively.

C. elegans strains and genetics. A list of strains used in this study is shown in Supplementary Table 6.

Drug treatment of C. elegans. All the C. elegans strains were maintained at $20^{\circ} \mathrm{C}$ in standard nematode growth medium (NGM) plates with OP50 as a food resource ${ }^{84}$. All the experiments were performed at $20^{\circ} \mathrm{C}$, unless specified elsewhere. DMSO-dissolved compounds (Kaem, Rhap, Quercetin, Ascomycin, Tacrolimus, Isorhamnetin and Pinostilbene) were added just before pouring the NGM plates. Worms were treated with designated drugs from either egg hatching or L4 stage, or other time points as specified elsewhere.

mRNA quantification using C. elegans tissue. Real-time PCR was performed as previously described ${ }^{85}$. Worms of designated age were collected, washed with M9 
buffer, followed by isolation of total RNA using TRIzol (Thermo Fisher, Catalog no. 15596026). For complementary DNA synthesis, messenger RNA was reversely transcribed using an iScript cDNA Synthesis Kit (catalog no. 1708890, Bio-Rad) for $5 \mathrm{~min}$ at $25^{\circ} \mathrm{C}, 20 \mathrm{~min}$ at $46^{\circ} \mathrm{C}, 1 \mathrm{~min}$ at $95^{\circ} \mathrm{C}$ and finally at $4{ }^{\circ} \mathrm{C}$ for storage. cDNA samples were then used for standard real-time quantitative reverse transcription PCR (real-time qRT-PCR) to quantify mRNA levels of $d c t-1$, pink-1, pdr-1, bec-1, sqst-1,vps-34, skn-1 and rheb-1 mRNA using the following primers:

dct-1: 5'-GGCTCCAACCTTACCACTCC- ${ }^{\prime}$ and $5^{\prime}$-GCAAATCCTACT GCTGCTCC-3';

pink-1: 5-AGCATATCGAATCGCAAATGAGTTAG-3' and

5'-TCGACCGTGGCGAGTTACAAG-3',

pdr-1: 5'-AGCCACCGAGCGATTGATTGC-3' and

5'-GTGGCATTTTGGGCATCTTCTTG-3';

bec-1: 5'-CTGTCAGCATCCGTTGAGGT-3' and

5'-AGAGCGTCAGAGCAATCATTACA-3';

sqst-1: 5'-ATCCGCTCCTCACCAAATGC-3' and

5'-TGTTGGACGAAGGGGAACAG-3';

$v p s-34: 5^{\prime}$-ATGATTCCAGGTATGCGGGC-3' and

5'-CTGACGAGCAAGTTGAGAGGA-3';

skn-1: ACAGTGCTTCTCTTCGGTAGC-3' and

5'-GAGACCCATTGGACGGTTGA-3'; and

rheb-1: 5'-ACAAGACGGATCTCAGCACG-3' and

5'-TCGAACACCTCATGCACTCG-3'.

Quantitative PCR was performed in triplicates, and the real-time PCR reactions were performed using QuantStudio 7 Flex System v1.1 (Applied Biosystems by Life Technologies) by heating to $95^{\circ} \mathrm{C}$ for $10 \mathrm{~min}$, cycling 40 times between $95^{\circ} \mathrm{C}$ for $15 \mathrm{~s}, 60^{\circ} \mathrm{C}$ for $1 \mathrm{~min}$, and taking a melt-curve analysis between $95^{\circ} \mathrm{C}$ for $15 \mathrm{~s}$ and $60^{\circ} \mathrm{C}$ for $1 \mathrm{~min}$

Evaluation of neuronal mitophagy inducers using a C. elegans mitophagy reporter strain. A C. elegans neuronal mitophagy reporter strain (neuronal mt-Rosella) was used to quantify neuronal mitophagy in worms as previously described: ${ }^{11}$ transgenic animals expressing a pan-neuronal mt-Rosella biosensor that combines a GFP variant sensitive to the acidic environment of the lysosomal lumen, fused to the $\mathrm{pH}$-insensitive Discosoma red fluorescent protein (DsRed) Mitophagy was calculated as GFP/DsRed. Thus, the lower the ratio of pixel intensity, the higher the level of mitophagy. For all nematode experiments, compounds $(0.01-1 \mathrm{mM})$ were administered from egg stage onwards. Unless specified, adult day 1 (the day after the L4 stage) nematodes were used for the experiments. Moreover, transgenic nematodes expressing the DCT-1::GFP mitophagy reporter, together with the autophagosomal marker LGG-1::DsRed in neurons were used to quantify mitophagy as previously reported ${ }^{11}$. Kaem and Rhap (0.01-1 mM) were administered from egg hatching onwards. Images of adult day 1 worms were taken using confocal microscopy, with co-localization between LGG-1 and DCT-1 serving to quantify the number of mitophagy events.

C. elegans short-term memory assay. Chemotaxis to soluble and volatile compounds (isoamyl alcohol, IA) was performed at $20^{\circ} \mathrm{C}$ on $10 \mathrm{~cm}$ agar plates as previously described ${ }^{11,86}$. In step 1, synchronized worms (around 200 worms per group) were collected and washed 5 times with M9 buffer, followed by placement in plain NGM plates (with no OP50) with/without IA for $90 \mathrm{~min}$. In this step, for the IA conditioning plate, a droplet of $10 \mu$ l pure IA was placed on the middle of the lid. In step 2, to prepare assay plates, $20 \mu \mathrm{l} 20 \mathrm{mM} \mathrm{NaN}_{3}$ was added on 'IA' and trap ' $T$ ' points, respectively. Assay plates were left at room temperature $\left(20-22^{\circ} \mathrm{C}\right)$ for $30 \mathrm{~min}$ to dry before testing. A $0.5 \times 0.5 \mathrm{~cm}$ Parafilm was placed over the 'IA' area of each plate. In step 3, worms from step 1 were collected via washing with M9 buffer, followed by placing the rinsed worms on the source point ' $S$ ' area (additional M9 buffer was quickly dried with tissue paper). Four $\mu$ l diluted IA (1/50) was added on the Parafilm placed on the 'IA' area. The worms were left at room temperature for $2 \mathrm{~h}$. For step 4, the number of worms in different regions were counted, including the ' $\mathrm{S}$ ', 'IA' and ' $\mathrm{T}$ ' areas. The chemotaxis index was calculated as (\#' $\mathrm{IA}-\#^{\prime} \mathrm{T}$ ')/ $\left(\#^{\prime} I A^{\prime}+\#^{\prime} T^{\prime}+\#^{\prime} S^{\prime}\right)$, where '\#' denotes numbers ${ }^{87}$. A smaller score correlates with better memory performance. Three to six biological replications were performed for all nematode experiments. The memory assay was performed using adult day 1 worms, unless specified elsewhere.

\section{C. elegans lifespan assay, pharyngeal pumping rate, mobility and toxicity} assays. Lifespan analysis was performed at $20^{\circ} \mathrm{C}$ as previously described ${ }^{88}$, using $3.5 \mathrm{~cm}$ NGM plates with $E$. coli OP50 seeded $3 \mathrm{~d}$ ahead of experiments. Drugs (final concentrations were $0.2 \mathrm{mM}$ Rhap, and $0.2 \mathrm{mM} \mathrm{Kaem}$ ) were added during pouring of the NGM plates. Synchronous animal populations were generated via a $6 \mathrm{~h}$ egg lay by gravid adults to obtain tightly synchronized embryos that were allowed to develop into adulthood under defined conditions. Around 35-40 L4 stage worms (day 0) were transferred to $3.5 \mathrm{~cm}$ plates to obtain synchronous populations of at least 100 animals per condition. Animals were scored as dead or alive and transferred every $2-3 \mathrm{~d}$ to fresh plates seeded with OP50 during the fertile period, and then every $5 \mathrm{~d}$ until death. Worms were examined every day and were considered dead when they had stopped pharyngeal pumping and were unresponsive to touch. Worms that died due to internal bagging, crawling on the edge of the plates or gonad extrusion were scored as censored. These animals were included in lifespan analyses up to the point of censorship and were weighted by ' 0 ' in mortality calculations. Parameters like mean, standard deviation of the mean and $P$ value were calculated using the log-rank test (Mantel-Cox) from a pooled population of animals. Kaplan-Meier (K-M) survival curves were used for lifespan presentation. We used the GraphPad Prism software package for statistical analysis and to determine lifespan values. For evaluations of pharyngeal pumping rates, worms were synchronized and raised to adults as mentioned in the lifespan assay methodology. At designated ages, pharynx contractions were manually counted for $30 \mathrm{~s}^{88}$. C. elegans movement analysis was performed as described ${ }^{89}$. Briefly, worms were exposed to designated concentrations of Kaem $(0.2 \mathrm{mM})$ and Rhap $(0.2 \mathrm{mM})$ beginning at egg stage and the maximum velocity of movement was determined at adult day 1 . Fifty to 100 nematodes were placed in one plate and recorded for $1 \mathrm{~h}$ and $30 \mathrm{~min}$ using the Movement Tracker system (Nemamtrix) to assess mobility. Three biological repeats were carried out. A double-blinded approach was used to ensure objectivity. Toxicity experiments, including fecundity (3 h egg-lay), egg hatching and larval development, were conducted using N2 C. elegans at $20^{\circ} \mathrm{C}$ as detailed elsewhere ${ }^{90}$. Briefly, synchronized eggs were placed on NGM plates seeded with E. coli (OP50). L4 larvae were subsequently transferred onto fresh OP50-seeded NGM plates and allowed to grow to adulthood. Ten adult day 1 worms were transferred onto assay NGM plates with OP50 and containing either $\operatorname{Kaem}(0.01,0.05,0.1,0.2,1 \mathrm{mM})$, Rhap $(0.01,0.05,0.1,0.2,1 \mathrm{mM})$ or vehicle control. Three plates of worms for each group were set up to achieve an $n=30$ per experimental condition. The gravid worms were subjected to a $3 \mathrm{~h}$ egg-lay followed by removal of worms from the plates. The number of eggs laid was quantified as a measure of the reproductive capacity of the worms. The following day, the number of unhatched eggs and L1 larvae were counted to determine the efficiency of egg hatching. Development to L4 larvae was assessed $36 \mathrm{~h}$ later after egg laying as a measure of larval growth. Finally, the growth of L4 larvae to adulthood was quantified $16 \mathrm{~h}$ after the L4 larval stage.

Evaluation of neuronal activity and neurodegeneration in C. elegans. Glutamatergic neurodegeneration was analysed using a well-established method reported elsewhere ${ }^{40,91}$. In brief, adult day 3 animals were prepared for confocal imaging. While there are around 15 glutamatergic neurons in the worm tail region $^{92}, 5$ were the focus of this analysis: LUA (R), LUA (L), PVR, PLM (R) and PLM (L). An animal was scored as normal if all 5 neurons were present and without malformations such as axon breaks, swelling, distension or separation of the soma. In addition, GFP intensity of the PVR neurons was quantified using the software Image J-1.50, with data from 30 worms randomly selected in each group from 2 biological replicates. Only PVR neurons with no clear malformations such as axon breaks, swelling, distension or abnormal location in the tail, were used for intensity quantification. The experiments were repeated 2 times with $40-50$ worms per group per experiment.

Cholinergic synaptic transmission assay was performed as previously described ${ }^{43}$. This assay evaluates the sensitivity of worms to the synaptic transmission of acetylcholine at the neuromuscular junction via monitoring the paralyzing effect of an acetylcholinesterase inhibitor, aldicarb. In the presence of aldicarb, breakdown of acetylcholine is inhibited, resulting in the accumulation of acetylcholine in the synaptic cleft. This build-up of acetylcholine results in over-activation of cholinergic receptors, followed by muscle hyper-contraction, paralysis and finally death. Briefly, synchronized adult day 1 worms, achieved via a $5 \mathrm{~h}$ egg-lay, were incubated in the presence of aldicarb at different concentrations and scored every $30 \mathrm{~min}$ for the time course of the aldicarb-induced paralysis. While a series of doses were used (vehicle, $0.25,0.5$ and $1 \mathrm{mM}$ ) for optimization, $0.5 \mathrm{mM}$ was chosen for further rescue experiments $( \pm 0.2 \mathrm{mM}$ Kaem or $0.2 \mathrm{mM}$ Rhap, or as described elsewhere). Thirty worms per group were used for the experiments, with finalized data from 2-4 biological repeats. VC223 (a strain hypersensitive to aldicarb-induced paralysis) and NM204 (a strain resistant to aldicarb-induced paralysis) were used as controls. Data were analysed and presented using Prism 8.0 (GraphPad Software).

Detection of in vitro mitophagy using mt-Keima fluorescent reporter. Imaging of mt-Keima HeLa cells was performed as reported elsewhere ${ }^{31}$, using different settings for GFP and red fluorescent protein (RFP). mt-Keima is a ratiometric $\mathrm{pH}$-sensitive fluorescent protein that exhibits green fluorescence (excitation $458 \mathrm{~nm}$ ) in basic or neutral conditions and red fluorescence (excitation $561 \mathrm{~nm}$ ) in acidic conditions $s^{33}$. For our experiments, the settings used were green channel (excitation $458 \mathrm{~nm}$, emission $570-695 \mathrm{~nm}$ ) to visualize normal mitochondria, and red channel (excitation $561 \mathrm{~nm}$, emission $570-695 \mathrm{~nm}$ ) to visualize mitochondria undergoing mitophagy. The mt-Keima HeLa cells were treated with different drugs of designated doses for $24 \mathrm{~h}$, followed by confocal imaging. A total of 5 randomly selected regions per sample was chosen for imaging, with a total of 3 biological repeats. Data were quantified, using ImageJ software, as the total number of red pixels divided by the total number of all pixels.

Evaluation of Tau aggregation and degradation in HEK293 cells expressed with P301S Tau-Venus. HEK293 cells stably expressing the 0N4R isoform of human Tau, bearing P301S mutation with a C-terminal Venus tag treated with exogenous recombinant heparin-assembled P301S Tau (tau seeds) were used for the experiments. Exogenous Tau seeds promote the conversion of endogenous 
Tau Venus from a dispersed distribution to bright foci ${ }^{49}$. Both the HEK293 P301S Tau-Venus cell line and the Tau seeds were a gift from the Dr William A. McEwan Lab at the University of Cambridge. The HEK293 P301S Tau-Venus cells were cultured in DMEM (with 10\% fetal bovine serum (FBS) and 1\% penicillinstreptomycin (P\&S)) in a humidified incubator at $37^{\circ} \mathrm{C}$ with $5 \% \mathrm{CO}_{2}$. On the day of the experiment, cells were trypsinized and seeded in Opti-MEM (Gibco, no FBS, no antibiotics) at a density of 5,000 cells per well per $50 \mu \mathrm{l}$ in a 96 -well plate (precoated with Poly d-lysine/PDL) and incubated for $24 \mathrm{~h}$. To induce endogenous Tau aggregation, $50 \mu \mathrm{l}$ Tau-containing Opti-MEM lacking FBS and antibiotics $(20 \mathrm{nM} \mathrm{Tau}+1 \%$ Lipofectamine 2000$)$ was pre-incubated at room temperature for $10 \mathrm{~min}$, then added to each well for $1.5 \mathrm{~h}$ incubation at $37^{\circ} \mathrm{C}$. Two types of experiments were performed: one to evaluate Tau aggregation and the other to quantify degradation of aggregated Tau. Experiment one was to evaluate how drugs affected seeded Tau-induced endogenous Tau aggregation: $50 \mu 1$ DMEM (with 20\% FBS and 1\% P\&S) containing vehicle (DMSO), Kaem $(3.125,6.25,12.5$, $25 \mu \mathrm{M})$ or Rhap $(3.125,6.25,12.5,25 \mu \mathrm{M})$ were added to respective wells, then incubated for $72 \mathrm{~h}$. Cells were then fixed with $4 \%$ formaldehyde solution in $1 \times$ PBS for $10 \mathrm{~min}$ at room temperature. Cells were then stained with DAPI $\left(1 \mu \mathrm{g} \mathrm{ml} \mathrm{m}^{-1}\right)$ in $1 \times$ PBS for $10 \mathrm{~min}$ before imaging. For experiment two, to evaluate how drugs affect degradation of aggregated Tau, $50 \mu \mathrm{L}$ DMEM (with 20\% FBS and 1\% P\&S) was added, followed by $48 \mathrm{~h}$ incubation. The medium was replaced with $150 \mu \mathrm{l}$ DMEM (with 10\% FBS and 1\% P\&S) and vehicle (DMSO), or Kaem $(3.125,6.25$, $12.5,25 \mu \mathrm{M})$ or Rhap $(3.125,6.25,12.5,25 \mu \mathrm{M})$ for $24 \mathrm{~h}$. Cells were fixed with $4 \%$ formaldehyde solution in PBS for $10 \mathrm{~min}$ at room temperature. Cells were stained with DAPI $\left(1 \mu \mathrm{g} \mathrm{ml}^{-1}\right)$ in PBS for $10 \mathrm{~min}$ before imaging. A fluorescent microscope (ZEISS Axio Zoom.V16) was used for imaging. One image per well was taken, with a total of 8 technical repeats per biological repeat. Data were analysed using ImageJ, from images from 3 biological repeats.

Cell culture for mechanistic studies. The N2a, YFP-Parkin HeLa, Mito-GFP/ mCherry-Parkin Hela, HEK 293 and GFP-LC3 HeLa cells were cultured in DMEM containing $10 \%$ FBS and $1 \%$ P\&S. N2S (N2a cells expressing human Swedish mutant APP695) and HEK 293 cells expressing pTRE3G-mcherry-BI promoter-EGFP Tau P301L were maintained in DMEM supplemented with $10 \% \mathrm{FBS}, 1 \% \mathrm{P} \& \mathrm{~S}$ and $200 \mu \mathrm{g} \mathrm{ml}^{-1} \mathrm{G} 418$. A high concentration (approximately $\left.1,000 \mu \mathrm{g} \mathrm{ml}^{-1}\right) \mathrm{G} 418$ was used for selection, and a low concentration $\left(200 \mu \mathrm{g} \mathrm{ml}^{-1}\right)$ was used for maintenance. The expression of EGFP-Tau P301L is controlled by the addition of doxycycline (DOX) to the culture medium before indicated treatments. All the cell lines were maintained in the incubator at $37^{\circ} \mathrm{C}$ with $5 \% \mathrm{CO}_{2}$. After designated treatments, cells were subjected to imaging (autophagy) or collected for western blot analysis. For the autophagy imaging, GFP-LC3 HeLa cells were fixed and stained with DAPI. The images were acquired using a confocal microscope (TCS SP8, Leica)

Transgenic mice, behavioural, pathological and mechanistic studies. Mice homozygous for all three mutant alleles (3xTg AD; homozygous for the Psen 1 mutation and homozygous for the co-injected APPSwe and tauP301L transgenes ( $\operatorname{Tg}($ APPSwe,tauP301L)1Lfa)) were obtained from the Jackson Laboratory. The 3xTg AD mice and age-matched C57 mice were housed in individually ventilated cages on standardized rodent bedding. Mice were housed under constant light cycle ( $12 \mathrm{~h}$ light/dark) with free food and water available. The 12.5 -month-old $3 \times \mathrm{Tg} \mathrm{AD}$ mice were treated with Kaem or Rhap $\left(100 \mathrm{mg} \mathrm{kg}^{-1} \mathrm{~d}^{-1}\right)$ by oral gavage for 2 months, and subsequently evaluated for behavioural and molecular endpoints. All animal care and experimental procedures were approved by the Committee on the Ethics of Animal Experiments of the University of Macau (UMARE-013-2019).

Mouse behavioural studies. Several behavioural assays, including Morris Water Maze (MWM), Y maze and NOR were used to investigate changes in learning and memory. The MWM test was performed as described previously ${ }^{93}$. The device is a circular white pool $(120 \mathrm{~cm}$ diameter $\times 50 \mathrm{~cm}$ depth $)$ filled with water dyed white with $\mathrm{TiO} 2$, and with temperature maintained at $22^{\circ} \mathrm{C}$. A $10-\mathrm{cm}$-diameter platform was placed $1 \mathrm{~cm}$ below the water surface at a fixed position. Mice were trained with 4 trials per day for 6 consecutive days. Each trial lasted $60 \mathrm{~s}$ or until the mouse found the platform. If the mouse did not find the platform during the allocated time period, the experimenter directed the mouse to the platform. After each trial, the mouse was placed on the platform for $10 \mathrm{~s}$. On the 7 th day, the platform was removed for a probe trial (60s) to assess long-term spatial memory retrieval. All parameters were recorded by a video tracking system (Labmaze V3.0, Zhongshi Technology). The Y maze spontaneous alternation performance (SAP) test measures the ability to recognize a previously explored environment ${ }^{94}$. The maze consists of $3 \mathrm{arms}$ ( $30 \mathrm{~cm}$ length $\times 20 \mathrm{~cm}$ height $\times 6 \mathrm{~cm}$ width) with an angle of $120^{\circ}$ between each arm. Mice were introduced to the centre of the Y maze and allowed to freely explore the maze for $10 \mathrm{~min}$. The maze was cleaned with $75 \%$ ethanol solution between animals to eliminate odour traces. The number of entries into each arm was recorded with Labmaze video tracking system. SAP is the number of subsequent forays into a novel arm over the course of 3 entries. The spontaneous alternation $(\%)=$ number of actual alternations/(total arm entries -2$) \times 100$. The NOR tests were performed as described previously
The device is a grey plastic box $(35 \mathrm{~cm}$ length $\times 35 \mathrm{~cm}$ width $\times 25 \mathrm{~cm}$ height $)$. Mice could explore two identical objects for 5 min during the training phase; $2 \mathrm{~h}$ later, each mouse was returned to the box, which had been modified to contain one familiar object and one novel object. The box was cleaned with $75 \%$ ethanol solution between animals to eliminate odour traces. The Labmaze video tracking system was used to monitor exploration behaviour. Exploration time was calculated as the length of time each mouse sniffed or pointed its nose or paws at the object. The 'recognition index' refers to the time spent exploring the novel object relative to the time spent exploring both objects. For the behavioural studies, 7 mice were used. No statistical methods were used to predetermine sample sizes, but our sample sizes are similar to those reported elsewhere ${ }^{96}$

EM. The ultrastructure of mitochondria and mitophagosomes was visualized and imaged with EM using the method reported elsewhere ${ }^{11}$. In brief, after killing the mice with the standard approach and a quick collection of the designated brain tissue, Veh-, Kaem- and Rhap-treated mouse hippocampal tissues were fixed in $2.5 \%$ glutaraldehyde ( $\mathrm{pH} 7.4$ ) for $2 \mathrm{~h}$. After three washes with $0.1 \mathrm{M}$ phosphate buffer ( $\mathrm{pH} 7.2$ ), the tissues were fixed in $1 \%$ osmic acid at $4{ }^{\circ} \mathrm{C}$ for $2 \mathrm{~h}$. The samples were gradient dehydrated in a series of ethanol baths. Subsequently, the samples were embedded in Epon-Araldite resin for penetration and placed in a mold for polymerization. After the semi-thin section was used for positioning, the ultra-thin section was made and collected for microstructure analysis, followed by counter-staining with $3 \%$ uranyl acetate and $2.7 \%$ lead citrate. The samples were then observed with an HT7800 transmission electron microscope. Three mice per group were used for EM examination, with 10-20 images randomly taken. Data were analysed manually in a double-blinded manner.

Immunohistochemistry/Immunofluorescence. After the behavioural tests, anaesthetized mice were perfused with pre-cooled $1 \times$ PBS. Half brains were placed in $4 \%$ paraformaldehyde in PBS for $24 \mathrm{~h}$ and then equilibrated in $30 \%$ sucrose for $72 \mathrm{~h}$ at $4{ }^{\circ} \mathrm{C} ; 1: 8$ series equidistant floating $30 \mu \mathrm{m}$ coronal sections $(240 \mu \mathrm{m}$ interval) were prepared, including the dentate gyrus area. Approximately 9-10 slices from each mouse were incubated in blocking buffer (5\% goat serum and $0.3 \%$ Triton X-100 in PBS) for $30 \mathrm{~min}$ at room temperature. Samples were then incubated overnight with the primary antibody at $4^{\circ} \mathrm{C}$ and then incubated with the appropriate fluorescent probe-conjugated secondary antibodies for $1 \mathrm{~h}$ at room temperature while protected from light. Nuclei were stained with DAPI at a $1: 1,000$ dilution. Specific primary antibodies used included mouse anti- $\beta$-amyloid, 1-16 antibody (clone 6E10, catalog no. 803002, BioLegend), rabbit anti-iba 1 antibody (019-19741, Wako) and mouse anti-AT8 antibody (cat no. MN1020, ThermoFisher). The slices were mounted with FluorSave reagent and visualized using the Leica TCS SP8 confocal microscope. A $\beta$ plaques and AT8-positive cells per ROI were counted and quantified using ImageJ software. To assess morphological changes of microglial cells, ImageJ was used to measure numbers and length of processes per microglial cell ${ }^{11,96}$. To evaluate phagocytosis, numbers of iba1-positive microglia cells that were also $\mathrm{A} \beta$-positive in the CA1 region were counted.

For the co-labelling analysis, rabbit anti-ibal antibody (019-19741, FUJIFILM Wako Pure Chemical) and mouse anti- $\beta$-amyloid, $1-16$ antibody (no. 803002 , BioLegend) were used. Primary antibodies were visualized with Alexa-Fluor 488 and Alex-Fluor 555 secondary antibodies (ThermoFisher), and the cell nuclei visualized with DAPI. For the quantification of $A \beta$ plaques, deposits larger than $10 \mu \mathrm{m}$ were included. At least 3 random images per mouse from a total of 3 mice were used for quantification. To detect the co-localization of mitochondria and lysosomes after Kaem and Rhap treatments, LAMP1 antibody (no. sc-18821, Santa Cruz) was used to label lysosomes in Mito-GFP/mCherry-Parkin HeLa cells. A Leica TCS SP8 confocal microscope was used to capture pictures and ImageJ software was used to quantify the co-localization events. To detect the effects of the top 5 potential compounds on mitophagy induction, HeLa cells expressing $\mathrm{mt}$-Keima were treated with designated compounds for $24 \mathrm{~h}$, then imaged and quantified with a Zeiss LSM 880 Confocal System.

Enzyme-linked immunosorbent assay (ELISA) for $\mathbf{A} \boldsymbol{\beta}_{1-40}$ and $\mathbf{A} \boldsymbol{\beta}_{1-42}$. ELISA assays were performed as reported previously, with minor modifications $s^{97}$. Mouse hippocampal tissues were homogenized individually with Qiagen TissueLyser-II homogenizer in radioimmunoprecipitation assay (RIPA) buffer (R0728, Sigma) containing protease and phosphatase inhibitors. Extracts were sonicated and spun at $100,000 \mathrm{~g}$ for $20 \mathrm{~min}$ at $4^{\circ} \mathrm{C}$. Insoluble pellets were extracted in $70 \%$ formic acid by sonication and spun at $100,000 \mathrm{~g}$ for $20 \mathrm{~min}$. Samples were neutralized in $1 \mathrm{M}$ Tris buffer ( $\mathrm{pH}$ 11, 1:12 ratio dilution) and diluted in blocking buffer before loading on ELISA. A $\beta$ in the RIPA fraction was regarded as the detergent-soluble fraction, while $A \beta$ in the neutralized formic acid fraction was considered as the detergent-insoluble fraction. The ELISA plates were coated overnight with $6 \mathrm{E} 10$ $\left(4 \mu \mathrm{g} \mathrm{ml}^{-1}\right)$ antibody in coating buffer $0.1 \mathrm{M} \mathrm{Na}_{2} \mathrm{CO}_{3}$ ( $\left.\mathrm{pH} 9.6\right)$ and blocked with $4 \%$ Block Ace in PBS for $2 \mathrm{~h}$. Equal amounts of the fractions were loaded in duplicate wells and incubated at room temperature for $2 \mathrm{~h}$ under shaking. Biotinylated 5C3 (no. 0060S, Nanotools) and 8G7 (no. 0061S, Nanotools) were used to determine $\mathrm{A} \beta_{1-40}$ and $\mathrm{A} \beta_{1-42}$, respectively. Secondary antibodies were diluted at 1:1,000 concentration in $1 \%$ Block Ace solution and incubated at room temperature for $2 \mathrm{~h}$ 
After washing the plates with PBS with $0.1 \%$ Tween 20 (PBST), streptavidin-HRP was added and the plates were incubated at $37^{\circ} \mathrm{C}$ for $1 \mathrm{~h}$. TMB substrate (no. 555214 , BD Biosciences) was then added to the plates, and plates were incubated at room temperature for $30 \mathrm{~min}$. Finally, an equal volume of stop solution was added, and absorbance was measured at $450 \mathrm{~nm}$.

Western blots using materials from cells and mouse brain. Western blot assay was done as previously described ${ }^{88}$. Samples from different cell lines and mouse brain tissues were collected and prepared using $1 \times$ RIPA buffer containing protease and phosphatase inhibitors, and the protein concentrations tested using the bovine serum albumin (BSA) method. Proteins were separated on a NuPAGE 4-12\% Bis-Tris Protein Gel at $200 \mathrm{~V}$ running for $1 \mathrm{~h}$ (catalogue no. NP0336BOX, Thermo Fisher Scientific) and set transfer system at $250 \mathrm{~mA}$ for $\sim 2-3 \mathrm{~h}$ (for all the proteins from $15 \mathrm{kDa}$ to $350 \mathrm{kDa}$ ) on PVDF membrane and then probed with various antibodies. Chemiluminescence detection was performed using a ChemiDoc XRS System (Bio-Rad Laboratories). Gamma adjustment was used to reduce the dark background when necessary. Quantification was performed using ImageJ. Antibodies used were as follows (all from Cell Signaling Technology, unless otherwise stated): PINK1 antibody (catalog no. ab75487, Abcam; no. A7131, ABclonal), Parkin antibody (no. NB100-91921, Novus), FUNDC1 antibody (no. ab74834, Abcam), LC3B antibody (no. NB100-2220, Novus), Beclin1 antibody (no. 3495s), phospho-DRP1 antibody (no. S616), DRP1 antibody (no. 8570s), p62 antibody (no. 8025s), MFN2 antibody (no. 94823s), phospho-ULK1 antibody (no. 5869s), ULK1 antibody (no. 6439s), AMBRA1 antibody (no. 24907s), OPTN antibody (no. A1845, ABclonal), Tau antibody (no. 46687s), p-Tau-thr181 (no. 12285s), p-Tau-thr231 (no. ab151559, Abcam), p-Tau-ser202/thr205 (no. MN1020, ThermoFisher), p-Tau-thr217 (no. 44-744, ThermoFisher), beta amyloid polyclonal antibody (no. 51-2700, ThermoFisher), Parkin Rabbit pAb (no. A0968, ABclonal), Tim 23 antibody (no. 611222, BD Biosciences), Total OXPHOS Rodent WB Antibody Cocktail (no. ab110413, Abcam) and $\beta$-actin antibody (no. A5441, Sigma). Secondary antibodies included anti-mouse immunoglobulin G (IgG; catalogue no. 7076s) and anti-rabbit IgG (no. 7074s). Primary antibodies were used at a 1:1,000 dilution, with secondary antibodies used at a 1:2,000-5,000 dilution.

Statistical analysis. Data are presented as mean \pm s.e.m., unless otherwise specified. Two-tailed unpaired $t$-tests were used for comparisons between two groups. Group differences were analysed with one-way analysis of variance (ANOVA) followed by Sidák's multiple comparisons test or two-way ANOVA followed by Tukey's multiple comparisons test for multiple groups. Prism 8.0 (GraphPad Software) was used for the statistical analysis. $P$ values $<0.05$ were considered statistically significant.

Reporting Summary. Further information on research design is available in the Nature Research Reporting Summary linked to this article.

\section{Data availability}

The main data supporting the findings of this study are available within the Article and its Supplementary Information. The raw wet-lab data generated in this study are available from the corresponding authors on reasonable request. Restrictions apply to the AI-related data: all requests for raw and processed data will be reviewed by Mindrank AI to verify whether the request is subject to any intellectual property or confidentiality constraints. Source data are provided with this paper.

\section{Code availability}

Custom code was specific to our computing infrastructure and mainly used for data input/output and parallelization across computers and graphics processors. The code, currently under a patent-examination process, is available from the corresponding authors on reasonable request.

Received: 20 February 2021; Accepted: 24 September 2021; Published online: 6 January 2022

\section{References}

1. Lou, G. et al. Mitophagy and neuroprotection. Trends Mol. Med. 26, 8-20 (2019).

2. Kerr, J. S. et al. Mitophagy and Alzheimer's disease: cellular and molecular mechanisms. Trends Neurosci. 40, 151-166 (2017).

3. Yuan, J. et al. Two conserved epigenetic regulators prevent healthy ageing. Nature 579, 118-122 (2020).

4. Kobro-Flatmoen, A. et al. Re-emphasizing early Alzheimer's disease pathology starting in select entorhinal neurons, with a special focus on mitophagy. Ageing Res. Rev. 67, 101307 (2021).

5. Lautrup, S., Sinclair, D. A., Mattson, M. P. \& Fang, E. F. NAD(+) in brain aging and neurodegenerative disorders. Cell Metab. 30, 630-655 (2019).

6. Patterson, C. World Alzheimer Report 2018: The State of the Art of Dementia Research: New Frontiers (ADI, 2019).

7. Cummings, J., Lee, G., Ritter, A., Sabbagh, M. \& Zhong, K. Alzheimer's disease drug development pipeline: 2019. Alzheimers Dement. 5, 272-293 (2019).
8. Mattson, M. P., Gleichmann, M. \& Cheng, A. Mitochondria in neuroplasticity and neurological disorders. Neuron 60, 748-766 (2008).

9. Tigano, M., Vargas, D. C., Tremblay-Belzile, S., Fu, Y. \& Sfeir, A. Nuclear sensing of breaks in mitochondrial DNA enhances immune surveillance. Nature 591, 477-481 (2021).

10. Aman, Y. et al. Autophagy in healthy aging and disease. Nat. Aging 1, 634-650 (2021)

11. Fang, E. F. et al. Mitophagy inhibits amyloid-beta and tau pathology and reverses cognitive deficits in models of Alzheimer's disease. Nat. Neurosci. 22 401-412 (2019).

12. Harold, D. et al. Genome-wide association study identifies variants at CLU and PICALM associated with Alzheimer's disease. Nat. Genet. 41, 1088-1093 (2009).

13. Lee, J. H. et al. Lysosomal proteolysis and autophagy require presenilin 1 and are disrupted by Alzheimer-related PS1 mutations. Cell 141, 1146-1158 (2010)

14. Fu, H. et al. A tau homeostasis signature is linked with the cellular and regional vulnerability of excitatory neurons to tau pathology. Nat. Neurosci. 22, 47-56 (2019).

15. Du, F. et al. PINK1 signalling rescues amyloid pathology and mitochondrial dysfunction in Alzheimer's disease. Brain 140, 3233-3251 (2017).

16. Brown, E. D. \& Wright, G. D. Antibacterial drug discovery in the resistance era. Nature 529, 336-343 (2016)

17. LeCun, Y., Bengio, Y. \& Hinton, G. Deep learning. Nature 521, 436-444 (2015).

18. Vamathevan, J. et al. Applications of machine learning in drug discovery and development. Nat. Rev. Drug Discov. 18, 463-477 (2019)

19. Stokes, J. M. et al. A deep learning approach to antibiotic discovery. Cell $\mathbf{1 8 0}$ 688-702 e613 (2020).

20. Mamoshina, P., Vieira, A., Putin, E. \& Zhavoronkov, A. Applications of deep learning in biomedicine. Mol. Pharm. 13, 1445-1454 (2016).

21. Chen, H., Engkvist, O., Wang, Y., Olivecrona, M. \& Blaschke, T. The rise of deep learning in drug discovery. Drug Discov. Today 23, 1241-1250 (2018).

22. Zhavoronkov, A. et al. Deep learning enables rapid identification of potent DDR1 kinase inhibitors. Nat. Biotechnol. 37, 1038-1040 (2019).

23. Rogers, D. \& Hahn, M. Extended-connectivity fingerprints. J. Chem. Inf. Model. 50, 742-754 (2010).

24. Gaulton, A. et al. The ChEMBL database in 2017. Nucleic Acids Res. 45 D945-D954 (2017).

25. Sterling, T. \& Irwin, J. J. ZINC 15-ligand discovery for everyone. J. Chem. Inf Model. 55, 2324-2337 (2015).

26. Jaeger, S., Fulle, S. \& Turk, S. Mol2vec: unsupervised machine learning approach with chemical intuition. J. Chem. Inf. Model. 58, 27-35 (2018).

27. Yuan, N. N. et al. Canthin-6-one accelerates alpha-synuclein degradation by enhancing UPS activity: drug target identification by CRISPR-Cas9 whole genome-wide screening technology. Front. Pharm. 10, 16 (2019).

28. Cai, C. Z. et al. Natural alkaloid harmine promotes degradation of alpha-synuclein via PKA-mediated ubiquitin-proteasome system activation. Phytomedicine 61, 152842 (2019).

29. Yang, X., Wang, Y., Byrne, R., Schneider, G. \& Yang, S. Concepts of artificial intelligence for computer-assisted drug discovery. Chem. Rev. 119, 10520-10594 (2019)

30. Pham, T. H., Qiu, Y., Zeng, J., Xie, L. \& Zhang, P. A deep learning framework for high-throughput mechanism-driven phenotype compound screening and its application to COVID-19 drug repurposing. Nat. Mach. Intell. 3, 247-257 (2021)

31. Sun, N. et al. Measuring in vivo mitophagy. Mol. Cell 60, 685-696 (2015).

32. Katayama, H., Kogure, T., Mizushima, N., Yoshimori, T. \& Miyawaki, A. A sensitive and quantitative technique for detecting autophagic events based on lysosomal delivery. Chem. Biol. 18, 1042-1052 (2011).

33. Fang, E. F. et al. In vitro and in vivo detection of mitophagy in human cells, C. elegans, and mice. J. Vis. Exp. 22, 56301 (2017).

34. Kingwell, K. Turning up mitophagy in Alzheimer disease. Nat. Rev. Drug Discov. https://www.nature.com/articles/d41573-019-00035-6 (2019).

35. Dosanjh, L. E., Brown, M. K., Rao, G., Link, C. D. \& Luo, Y. Behavioral phenotyping of a transgenic Caenorhabditis elegans expressing neuronal amyloid-beta. J. Alzheimers Dis. 19, 681-690 (2010).

36. Fang, E. F. et al. Tomatidine enhances lifespan and healthspan in C. elegans through mitophagy induction via the SKN-1/Nrf2 pathway. Sci. Rep. 7, 46208 (2017).

37. Lazarou, M. et al. The ubiquitin kinase PINK1 recruits autophagy receptors to induce mitophagy. Nature 524, 309-314 (2015).

38. Fang, E. F. et al. Defective mitophagy in XPA via PARP-1 hyperactivation and NAD(+)/SIRT1 reduction. Cell 157, 882-896 (2014).

39. Francis, P. T. The interplay of neurotransmitters in Alzheimer's disease. CNS Spectr. 10, 6-9 (2005).

40. Griffin, E. F. et al. ApoE-associated modulation of neuroprotection from Abeta-mediated neurodegeneration in transgenic Caenorhabditis elegans. Dis. Model Mech. 12, dmm037218 (2019). 
41. Treusch, S. et al. Functional links between Abeta toxicity, endocytic trafficking, and Alzheimer's disease risk factors in yeast. Science 334, 1241-1245 (2011).

42. Hampel, H. et al. The cholinergic system in the pathophysiology and treatment of Alzheimer's disease. Brain 141, 1917-1933 (2018).

43. Mahoney, T. R., Luo, S. \& Nonet, M. L. Analysis of synaptic transmission in Caenorhabditis elegans using an aldicarb-sensitivity assay. Nat. Protoc. 1, 1772-1777 (2006).

44. Cummins, N., Tweedie, A., Zuryn, S., Bertran-Gonzalez, J. \& Gotz, J. Disease-associated tau impairs mitophagy by inhibiting Parkin translocation to mitochondria. EMBO J. e99360 (2018)

45. Fatouros, C. et al. Inhibition of tau aggregation in a novel Caenorhabditis elegans model of tauopathy mitigates proteotoxicity. Hum. Mol. Genet. 21 3587-3603 (2012).

46. Iba, M. et al. Synthetic tau fibrils mediate transmission of neurofibrillary tangles in a transgenic mouse model of Alzheimer's-like tauopathy. J. Neurosci. 33, 1024-1037 (2013).

47. Spillantini, M. G. \& Goedert, M. Tau pathology and neurodegeneration. Lancet Neurol. 12, 609-622 (2013).

48. Wegmann, S. et al. Experimental evidence for the age dependence of tau protein spread in the brain. Sci. Adv. 5, eaaw6404 (2019).

49. McEwan, W. A. et al. Cytosolic Fc receptor TRIM21 inhibits seeded tau aggregation. Proc. Natl Acad. Sci. USA 114, 574-579 (2017).

50. Wolfson, C. et al. A reevaluation of the duration of survival after the onset of dementia. N. Engl. J. Med. 344, 1111-1116 (2001).

51. Kua, E. H. et al. The natural history of dementia. Psychogeriatrics 14, 196-201 (2014).

52. Cabreiro, F. et al. Metformin retards aging in C. elegans by altering microbial folate and methionine metabolism. Cell 153, 228-239 (2013).

53. Ryu, D. et al. Urolithin A induces mitophagy and prolongs lifespan in C. elegans and increases muscle function in rodents. Nat. Med. 22, 879-888 (2016).

54. Oddo, S. et al. Triple-transgenic model of Alzheimer's disease with plaques and tangles: intracellular Abeta and synaptic dysfunction. Neuron 39, 409-421 (2003).

55. Lautrup, S. et al. Microglial mitophagy mitigates neuroinflammation in Alzheimer's disease. Neurochem. Int. 129, 104469 (2019).

56. Mattsson-Carlgren, N. et al. Longitudinal plasma p-tau217 is increased in early stages of Alzheimer's disease. Brain 143, 3234-3241 (2020).

57. Palmqvist, S. et al. Discriminative accuracy of plasma phospho-tau217 for Alzheimer disease vs other neurodegenerative disorders. JAMA $\mathbf{3 2 4}$, 772-781 (2020).

58. Aman, Y. et al. The $\mathrm{NAD}(+)$-mitophagy axis in healthy longevity and in artificial intelligence-based clinical applications. Mech. Ageing Dev. 185, 111194 (2020)

59. Zhu, T. et al. Hit identification and optimization in virtual screening: practical recommendations based on a critical literature analysis. J. Med. Chem. 56, 6560-6572 (2013).

60. Truchon, J. F. \& Bayly, C. I. Evaluating virtual screening methods: good and bad metrics for the "early recognition" problem. J. Chem. Inf. Model. 47, 488-508 (2007).

61. Chuang, K. V., Gunsalus, L. \& Keiser, M. J. Learning molecular representations for medicinal chemistry. J. Med. Chem. 63, 8705-8722 (2020)

62. Stokes, J. M. et al. A deep learning approach to antibiotic discovery. Cell 181, 475-483 (2020).

63. Holland, T. M. et al. Dietary flavonols and risk of Alzheimer dementia. Neurology 94, e1749-e1756 (2020).

64. Pollack, R. M. et al. Resveratrol improves vascular function and mitochondrial number but not glucose metabolism in older adults. J. Gerontol. A Biol. Sci. Med Sci. 72, 1703-1709 (2017).

65. Bonkowski, M. S. \& Sinclair, D. A. Slowing ageing by design: the rise of $\mathrm{NAD}(+)$ and sirtuin-activating compounds. Nat. Rev. Mol. Cell Biol. 17, 679-690 (2016).

66. Kouhestani, S., Jafari, A. \& Babaei, P. Kaempferol attenuates cognitive deficit via regulating oxidative stress and neuroinflammation in an ovariectomized rat model of sporadic dementia. Neural Regen. Res. 13, 1827-1832 (2018).

67. Kim, J. K. et al. Protective effects of kaempferol (3,4,5,7-tetrahydroxyflavone) against amyloid beta peptide (Abeta)-induced neurotoxicity in ICR mice. Biosci. Biotechnol. Biochem. 74, 397-401 (2010).

68. Zhang, Q. et al. Improved blood-brain barrier distribution: effect of borneol on the brain pharmacokinetics of kaempferol in rats by in vivo microdialysis sampling. J. Ethnopharmacol. 162, 270-277 (2015).

69. Moradi-Afrapoli, F. et al. Validation of UHPLC-MS/MS methods for the determination of kaempferol and its metabolite 4-hydroxyphenyl acetic acid, and application to in vitro blood-brain barrier and intestinal drug permeability studies. J. Pharm. Biomed. Anal. 128, 264-274 (2016).

70. Roupe, K. A., Yanez, J. A., Teng, X. W. \& Davies, N. M. Pharmacokinetics of selected stilbenes: rhapontigenin, piceatannol and pinosylvin in rats. J. Pharm. Pharmacol. 58, 1443-1450 (2006).
71. Stallings, N. R. et al. Pin1 mediates Abeta42-induced dendritic spine loss. Sci. Signal 11, eaap8734 (2018)

72. Musuamba, F. T. et al. Advanced methods for dose and regimen finding during drug development: summary of the EMA/EFPIA workshop on dose finding (London 4-5 December 2014). CPT Pharmacometrics Syst. Pharmacol. 6, 418-429 (2017).

73. Baell, J. B. \& Holloway, G. A. New substructure filters for removal of pan assay interference compounds (PAINS) from screening libraries and for their exclusion in bioassays. J. Med. Chem. 53, 2719-2740 (2010).

74. Dahlin, J. L. et al. PAINS in the assay: chemical mechanisms of assay interference and promiscuous enzymatic inhibition observed during a sulfhydryl-scavenging HTS. J. Med. Chem. 58, 2091-2113 (2015).

75. Baell, J. B. \& Nissink, J. W. M. Seven year itch: pan-assay interference compounds (PAINS) in 2017 - utility and limitations. ACS Chem. Biol. 13, 36-44 (2018).

76. Baell, J. \& Walters, M. A. Chemistry: chemical con artists foil drug discovery. Nature 513, 481-483 (2014)

77. Hughes, J. P., Rees, S., Kalindjian, S. B. \& Philpott, K. L. Principles of early drug discovery. Br. J. Pharmacol. 162, 1239-1249 (2011).

78. Yoshino, M. et al. Nicotinamide mononucleotide increases muscle insulin sensitivity in prediabetic women. Science 372, 1224-1229 (2021).

79. Cen, X. et al. Pharmacological targeting of MCL-1 promotes mitophagy and improves disease pathologies in an Alzheimer's disease mouse model. Nat. Commun. 11, 5731 (2020).

80. Fang, E. F. Mitophagy and $\mathrm{NAD}(+)$ inhibit Alzheimer disease. Autophagy 15, 1112-1114 (2019).

81. Landrum, G. RDKit: A software suite for cheminformatics, computational chemistry, and predictive modeling, Academic Press, https://doi.org/10.15252/ embj.201899360 (2013).

82. Ehrlich, P. Über den jetzigen Stand der Chemotherapie. Ber. Dtsch. Chem. Ges. 42, 17-47 (1909).

83. Landrum, G. A., Penzotti, J. E. \& Putta, S. Feature-map vectors: a new class of informative descriptors for computational drug discovery. J. Comput. Aided Mol. Des. 20, 751-762 (2006).

84. Brenner, S. The genetics of Caenorhabditis elegans. Genetics 77, 71-94 (1974).

85. Fang, E. F. et al. NAD(+) augmentation restores mitophagy and limits accelerated aging in Werner syndrome. Nat. Commun. 10, 5284 (2019).

86. Voglis, G. \& Tavernarakis, N. A synaptic DEG/ENaC ion channel mediates learning in C. elegans by facilitating dopamine signalling. EMBO J. 27, 3288-3299 (2008).

87. Bargmann, C. I. \& Horvitz, H. R. Chemosensory neurons with overlapping functions direct chemotaxis to multiple chemicals in C. elegans. Neuron 7, 729-742 (1991).

88. Fang, E. F. et al. NAD(+) replenishment improves lifespan and healthspan in ataxia telangiectasia models via mitophagy and DNA repair. Cell Metab. 24, 566-581 (2016)

89. Sorrentino, V. et al. Enhancing mitochondrial proteostasis reduces amyloid-beta proteotoxicity. Nature 552, 187-193 (2017).

90. Ianevski, A. et al. Chemical, physical and biological triggers of evolutionary conserved Bcl-xL-mediated apoptosis. Cancers 12, 1694 (2020).

91. Matlack, K. E. et al. Clioquinol promotes the degradation of metal-dependent amyloid-beta (Abeta) oligomers to restore endocytosis and ameliorate Abeta toxicity. Proc. Natl Acad. Sci. USA 111, 4013-4018 (2014).

92. Serrano-Saiz, E. et al. Modular control of glutamatergic neuronal identity in C. elegans by distinct homeodomain proteins. Cell 155, 659-673 (2013).

93. Vorhees, C. V. \& Williams, M. T. Morris water maze: procedures for assessing spatial and related forms of learning and memory. Nat. Protoc. 1, 848-858 (2006).

94. Ghosal, K. et al. Alzheimer's disease-like pathological features in transgenic mice expressing the APP intracellular domain. Proc. Natl Acad. Sci. USA 106, 18367-18372 (2009).

95. Leger, M. et al. Object recognition test in mice. Nat. Protoc. 8 , 2531-2537 (2013)

96. Iaccarino, H. F. et al. Gamma frequency entrainment attenuates amyloid load and modifies microglia. Nature 540, 230-235 (2016).

97. Song, J. X. et al. A small molecule transcription factor EB activator ameliorates beta-amyloid precursor protein and Tau pathology in Alzheimer's disease models. Aging Cell 19, e13069 (2020).

\section{Acknowledgements}

This project was supported by HELSE SØR-ØST (Nos. 2017056, 2020001 and 2021021), the Research Council of Norway (Nos. 262175 and 277813), the National Natural Science Foundation of China (No. 81971327), Akershus University Hospital (Nos. 269901 and 261973), the Civitan Norges Forskningsfond for Alzheimers sykdom (for a 3-year Ph.D. Fellowship, No. 281931) and the Czech Republic-Norway KAPPA programme (with Martin Vyhnálek, No. TO01000215) to E.F.F. The project was partially supported by the Science and Technology Development Fund, Macau SAR (Grants No. 0128/2019/ A3 and 024/2017/AMJ), the University of Macau grants (Grant No. MYRG201900129-ICMS) awarded to J.H.L. H.N. was supported by grants from HELSE SØR-ØST 
(No. 2019098) and the Research Council of Norway (No. 302483). G.Y. was supported by the British Heart Foundation (Project No. PG/16/78/32402), the Hangzhou Economic and Technological Development Area Strategical Grant (Imperial Institute of Advanced Technology), the European Research Council Innovative Medicines Initiative on Development of Therapeutics and Diagnostics Combatting Coronavirus Infections Award 'DRAGON: rapiD and secuRe AI imaging-based diaGnosis, stratification, fOllow-up and preparedness for coronavirus paNdemics' (H2020-JTI-IMI2 101005122), the AI for Health Imaging Award 'CHAIMELEON: Accelerating the Lab to Market Transition of AI Tools for Cancer Management' (H2020-SC1-FA-DTS-2019-1 952172) and the UK Research and Innovation Future Leaders Fellowship (MR/V023799/1). C.X. was supported by the National Science Foundation of China (No. 81600977), Wenzhou City Committee of Science and Technology (Y20180137) and the Natural Science Foundation of Zhejiang Province (Y19H090059). R.A. and H.W. were funded by the China Scholarship Council (http://www.csc.edu.cn/); the funders had no role in study design, data collection and analysis, decision to publish or preparation of the manuscript. Support for some of the transgenic nematode models used came from a Collaborative Innovation Award from the Howard Hughes Medical Institute to G.A.C. We thank B. Olaisen (KCL) and D. J. Patrick-Brown (UiO) for reading and comments on the manuscript. Cartoon images in Fig. 2a and Supplementary Fig. 4a were generated using BioRender via subscription through the Fang laboratory.

\section{Author contributions}

E.F.F. designed and coordinated the project. C.X., R.A., S.L., R.H., T.S.G., M.J.L.-D., D.C., W.-W.W., T.S.-M., J.Z., H.W., G.L., K.P. and H.N. did the worm experiments. K.A.C. and G.A.C. generated the worm strains and helped in data analysis on neurodegeneration. X.-X.Z., C.-Z.C., L.-M.X., W.Z. and J.-H.L. did the mouse experiments. S.C. did the Tau seeding experiments. Z.N., S.Z., Y.J., G.Y. and X.X. did the AI experiments. C.X. and E.F.F. wrote the manuscript, while X.-X.Z., Z.N., J.-H.L., K.A.C., G.A.C., H.N., K.P., H.M.S. and G.Y. edited the manuscript.

\section{Competing interests}

E.F.F. and H.N. have a cooperative research and development agreement with ChromaDex. E.F.F., G.Y. and H.N. are consultants to Aladdin Healthcare
Technologies. E.F.F. is a consultant to the Vancouver Dementia Prevention Centre and Intellectual Labs. During the execution of this work, S.Z. was a paid part-time employee of MindRank Al Ltd. Z.N., Y.J. and X.X. are full-time employees of MindRank Al Ltd. E.F.F., J.-H.L, Z.N., C.X. and X.-X.Z. have filed a patent on the use of mitophagy inducers to treat Alzheimer's disease. The other authors declare no competing interests.

\section{Additional information}

Extended data is available for this paper at https://doi.org/10.1038/s41551-021-00819-5.

Supplementary information The online version contains supplementary material available at https://doi.org/10.1038/s41551-021-00819-5.

Correspondence and requests for materials should be addressed to Jia-Hong Lu or Evandro F. Fang.

Peer review information Nature Biomedical Engineering thanks Thomas Caulfield, Feixiong Cheng and Hongguang Xia for their contribution to the peer review of this work.

Reprints and permissions information is available at www.nature.com/reprints. Publisher's note Springer Nature remains neutral with regard to jurisdictional claims in published maps and institutional affiliations.

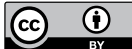

Open Access This article is licensed under a Creative Commons Attribution 4.0 International License, which permits use, sharing, adaptation, distribution and reproduction in any medium or format, as long as you give appropriate credit to the original author(s) and the source, provide a link to the Creative Commons license, and indicate if changes were made. The images or other third party material in this article are included in the article's Creative Commons license, unless indicated otherwise in a credit line to the material. If material is not included in the article's Creative Commons license and your intended use is not permitted by statutory regulation or exceeds the permitted use, you will need to obtain permission directly from the copyright holder. To view a copy of this license, visit http://creativecommons. org/licenses/by/4.0/.

(C) The Author(s) 2022 


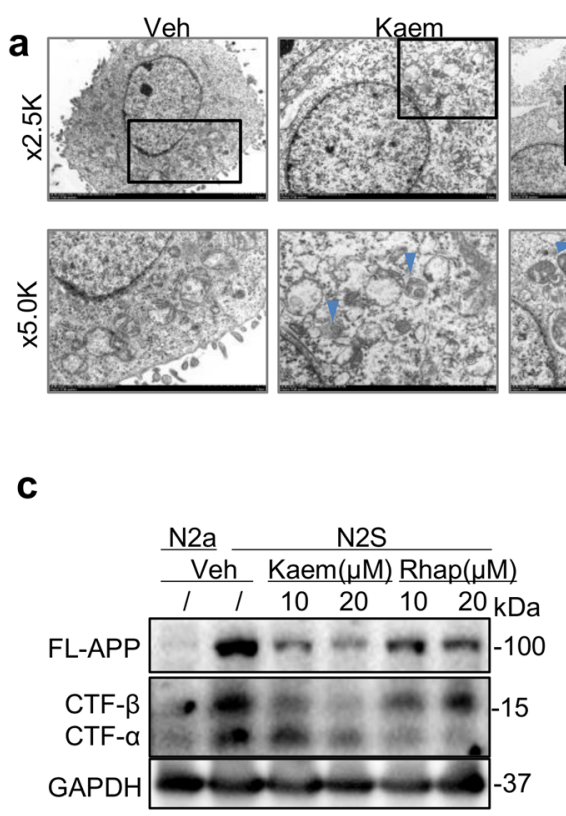

Rhap

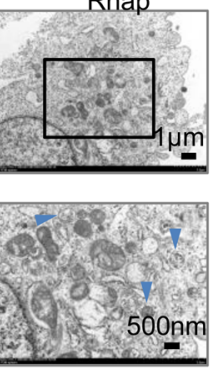

b

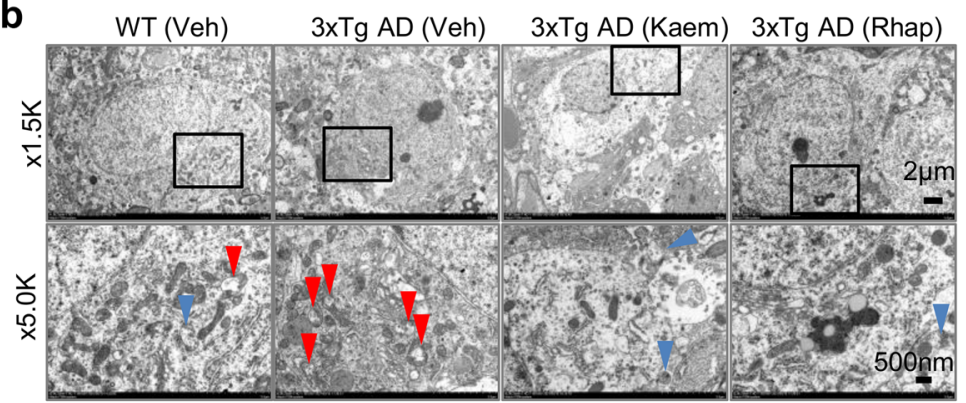

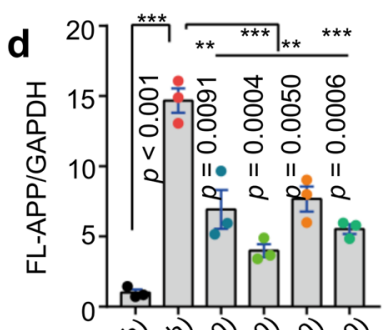

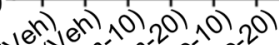

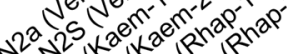

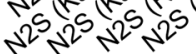

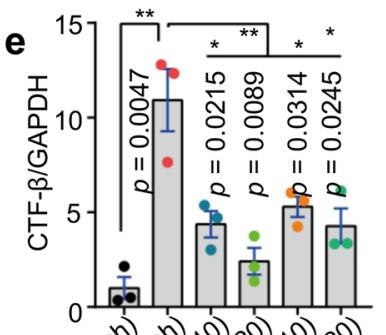

(e)

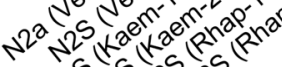
${ }^{2} \mathrm{a}^{2} \mathrm{n}^{2} \mathrm{a}^{5}$

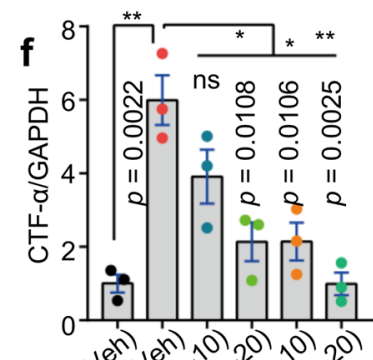

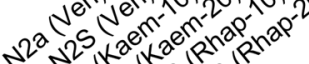
$35 \sqrt{5} \mathrm{a}^{5} \mathrm{n}^{5}$
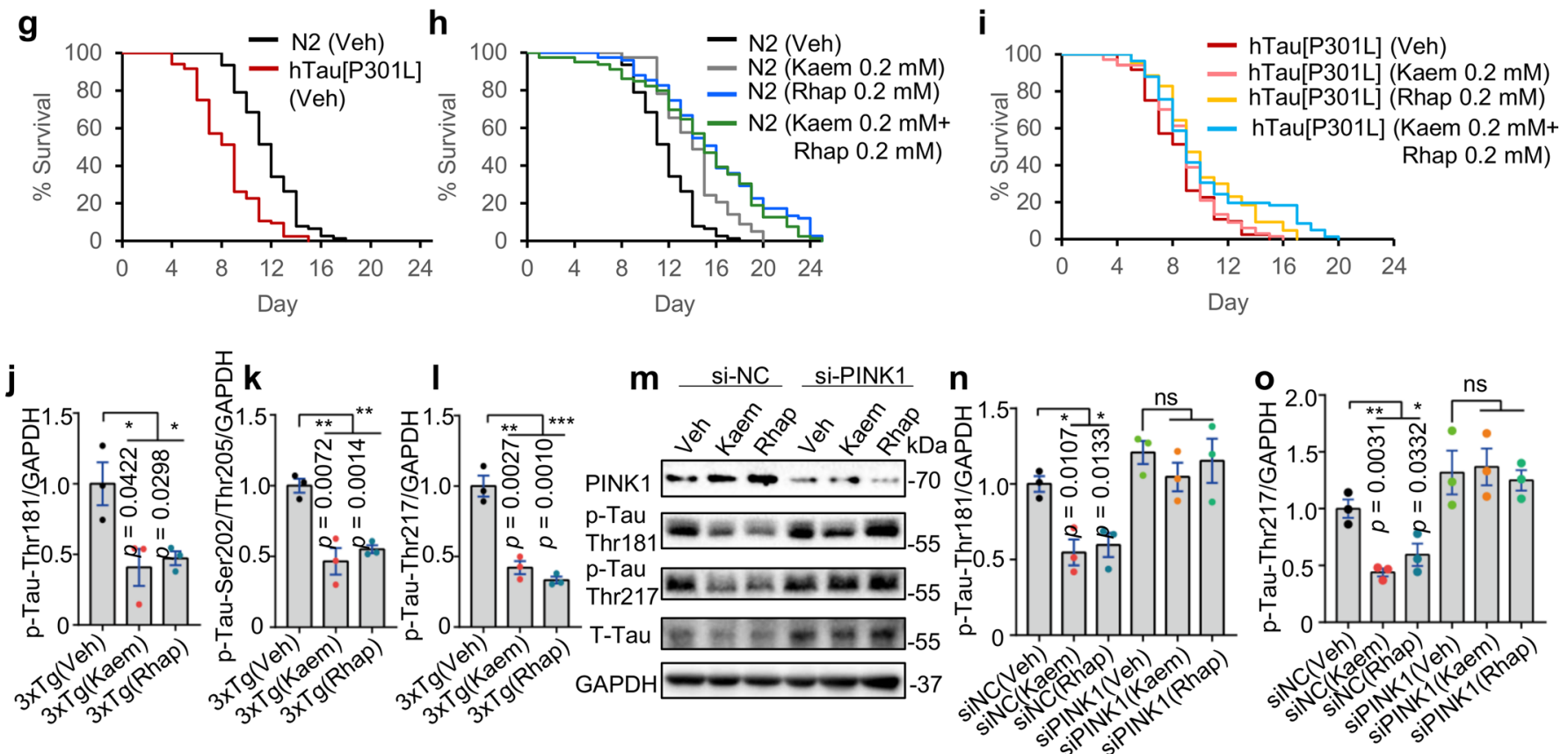

Extended Data Fig. 1 | See next page for caption. 
Extended Data Fig. 1 | Kaem and Rhap induce mitophagy, abrogate disease pathologies, and improve healthspan and lifespan in cells, worm, or mouse models of AD. $\mathbf{a}$, $\mathbf{b}$, electron microscopic images showing effects of Kaem and Rhap on mitochondrial morphology and mitophagy-like events in mt-Keima HeLa cells ( $\mathrm{a}, 20 \mu \mathrm{M}$ for $24 \mathrm{~h}$ ) and mouse hippocampal brain tissues (b, $100 \mathrm{mg} / \mathrm{kg} / \mathrm{d}$ via oral gavage from 12 months for 7 consecutive days; $\mathrm{n}=3 \mathrm{mice}$ per group with 4 random hippocampal neuronal images per mouse), treated with Kaem and Rhap. Blue arrows indicate mitophagosome-like events, while red arrows point to damaged mitochondria. Quantitative data of a and b are shown in Fig. 3m and Fig. 3n, respectively. c-f, Effects of Kaem and Rhap on the expression of designated proteins involved in A $\beta$ production. N2a and N2S (N2a stably transfected with human Swedish mutant APP695) cells were used. One set of western blot data is shown (c), with semi-quantifications from three biological replicates (d-f). All quantitative data were shown in mean \pm S.E.M. One-way ANOVA followed by Šidák's multiple comparisons test (d-f) were used for data analysis. NS, no significance, ${ }^{\star} p<0.05,{ }^{\star \star} p<0.01$, ${ }^{\star \star \star} p<0.001$. Original western blot gels for (c) are included in Source Data Fig. 2. g-i, Effects of Kaem and Rhap on lifespan of the N2 and hTau[P301L] (CK12) worms. Data shown are from one set of experiments from a total of two biological replicates (quantitative values in Supplementary Table 4). 90-120 worms were used for each group/biological repeat. All quantitative data were shown in mean \pm S.E.M. Log-rank test was used for the statistics of lifespan data (g-i). NS, no significance, ${ }^{\star} p<0.05,{ }^{\star \star} p<0.01,{ }^{\star \star \star} p<0.001$. j-I, Quantification of phosphorylated Tau sites (Thr181, Ser202/Thr205, Thr217) in hippocampal tissues from the $3 \times \operatorname{Tg} A D$ mice $(n=3$ biological replicates). The western blotting gel data are shown in Fig. 60. m-o, Effects of PINK1 knockdown on p-Tau inhibition by Kaem and Rhap in HEK 293 3G-EGFP-Tau P301L/mCherry cells. While one biological set of blot data are shown (m), quantifications $(\mathbf{n}, \mathbf{0})$ were from 3 biological replicates. All quantitative data were shown in mean \pm S.E.M. Two-way ANOVA followed by Tukey's multiple comparisons test $(\mathbf{j}, \mathbf{k} . \mathbf{I})$ and one-way ANOVA followed by Šidák's multiple comparisons test (n, o) were used for data analysis. NS, no significance, ${ }^{\star} p<0.05,{ }^{\star \star} p<0.01,{ }^{\star \star \star} p<0.001$. Original western blot gels for $(\mathbf{m})$ were included in Source Data Fig. 2. 


\section{HeLa cells}
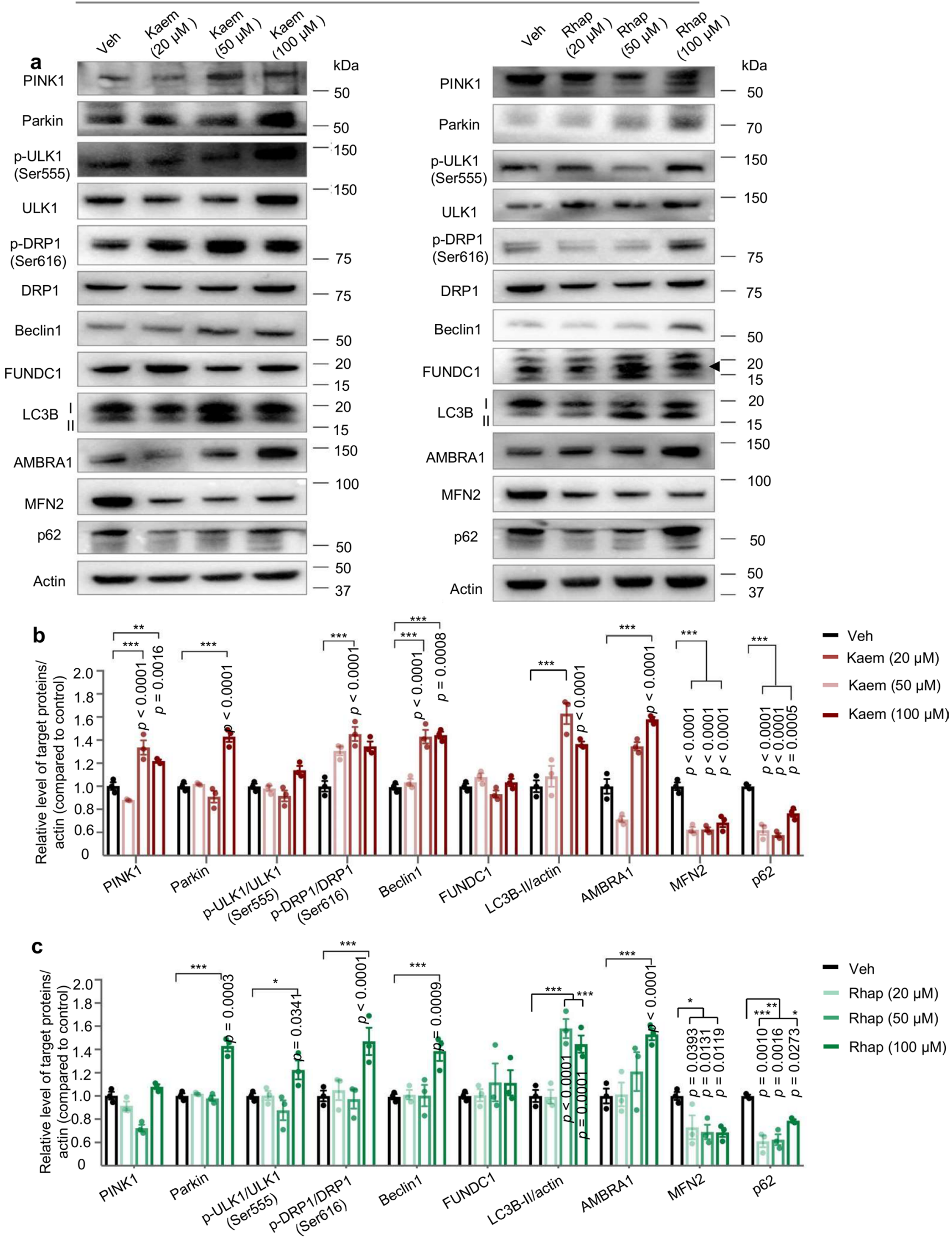

Extended Data Fig. 2 | See next page for caption. 
Extended Data Fig. 2 | Kaem and Rhap induce expression of a broad spectrum of autophagy/mitophagy proteins. a, A representative set of western

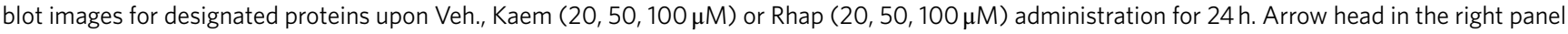
pointed to 'FUNDC1', with bands upper and lower were likely unknown or non-specific bands. $\mathbf{b}$, c, Semi-quantification of $\mathbf{a}$ from three biological replicates with data shown in mean \pm S.E.M. One-way ANOVA followed by Šidák's multiple comparisons test was used for data analysis, with ${ }^{\star} p<0.05,{ }^{\star \star} p<0.01$, ${ }^{\star \star \star} p<0.001$. Original western blot gels for (a) were included in Source Data Fig. 1. 


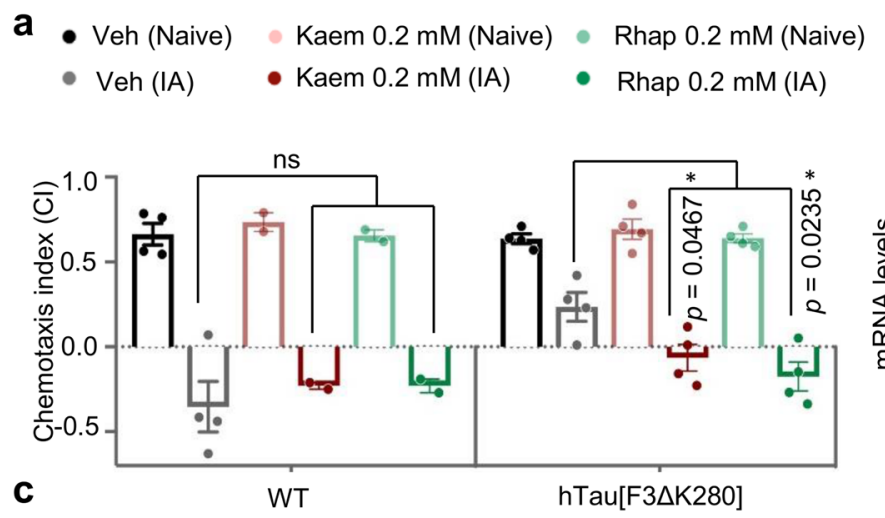

Same legend as in a

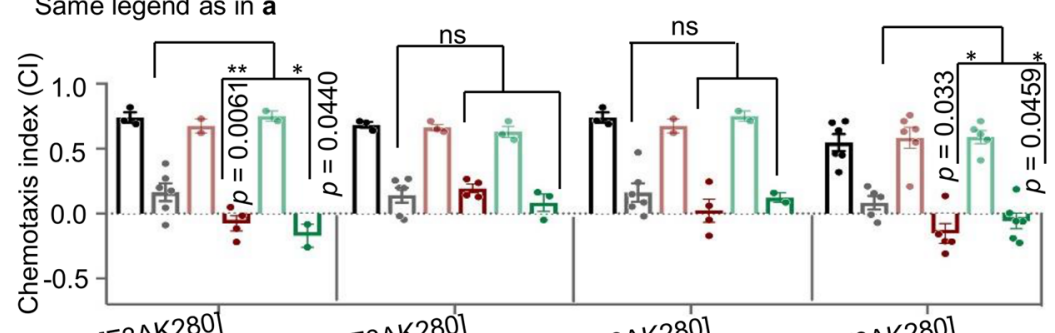

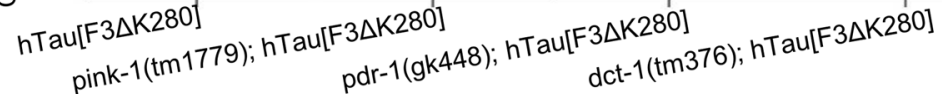

e Same legend as in a
With UV killed OP50

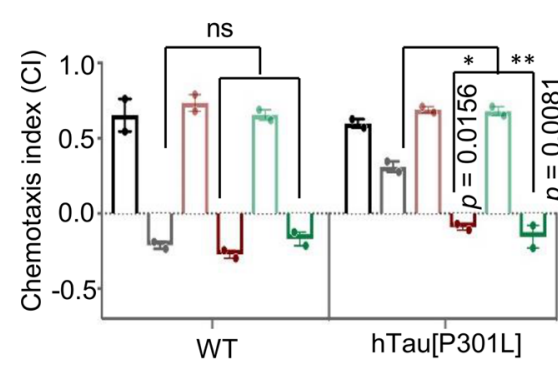

960 mins ns

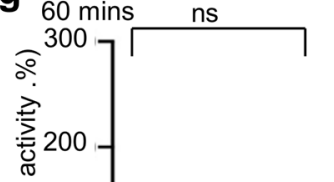

$\frac{\pi}{\overline{\bar{N}}}$

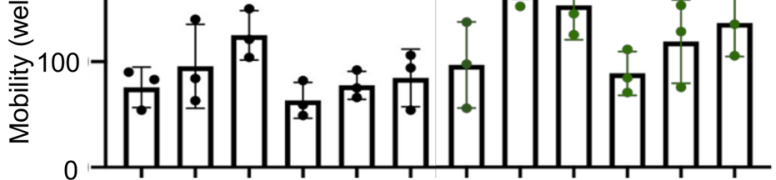

हe
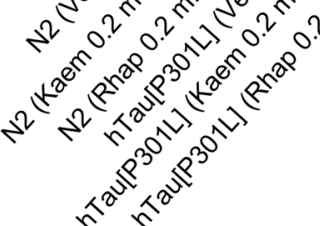

$$
\text { f }
$$

- N2 (Kaem 0.2 mM) • hTau[P301L] (Kaem 0.2 mM)

- N2 (Rhap 0.2 mM) • hTau[P301L] (Rhap 0.2 mM)

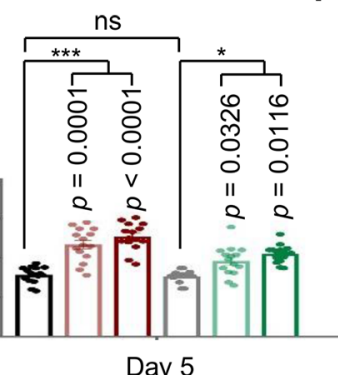

h
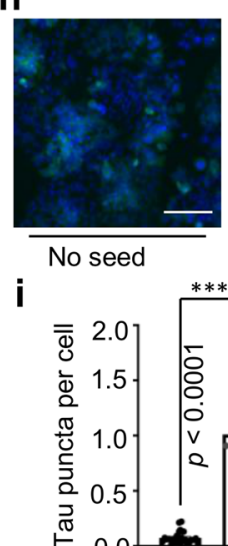

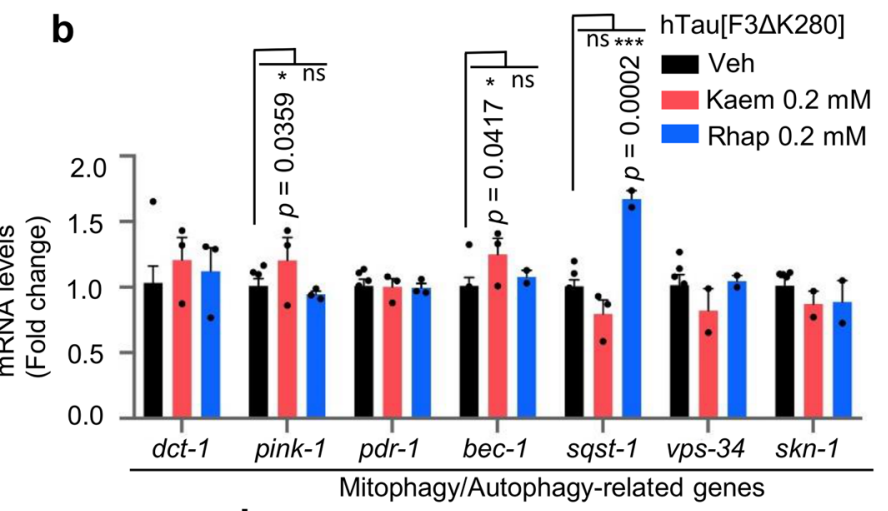

d

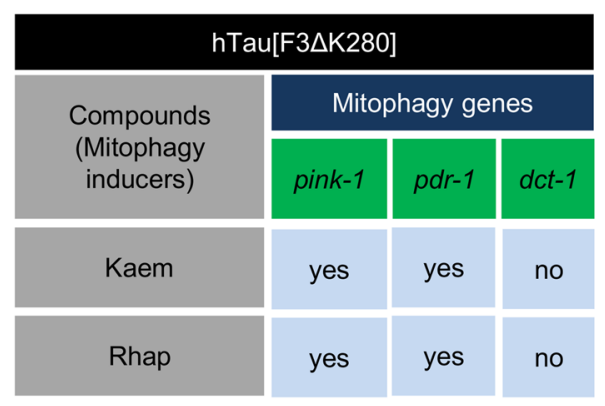

j

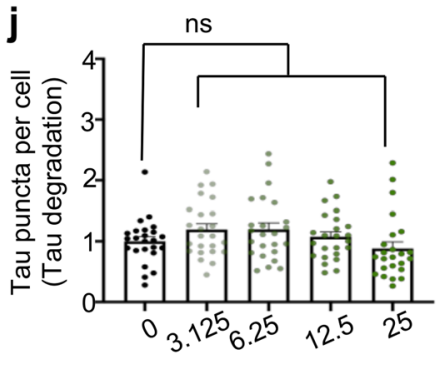

Tau-seed $(10 \mathrm{nM})+$ Kaem $(\mu \mathrm{M})$

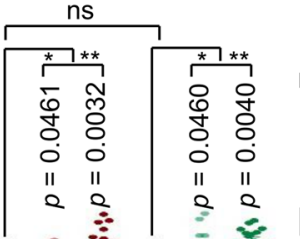

k

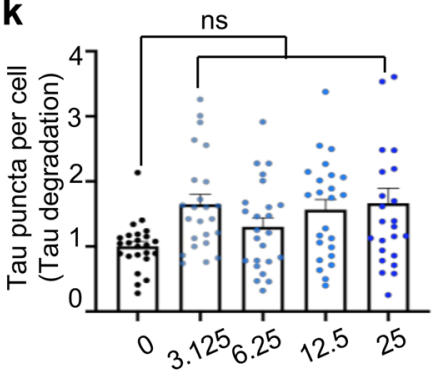

Tau-seed $(10 \mathrm{nM})+$ Rhap $(\mu \mathrm{M})$

Tau P301S (seeds)

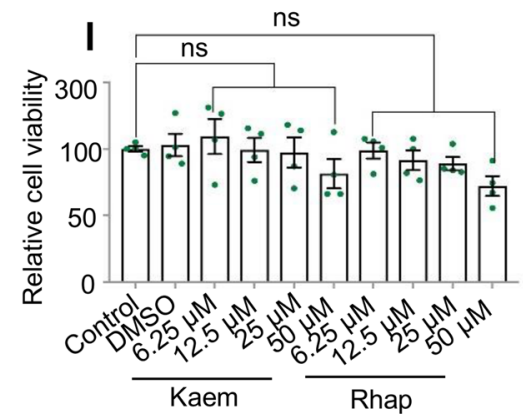

Extended Data Fig. 3 | See next page for caption. 


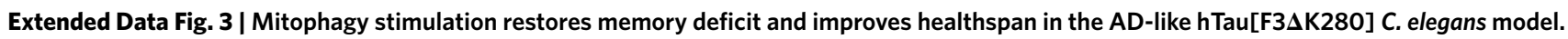
a, Effects of Kaem and Rhap on associative memory in transgenic animals expressing hTau[F3 $\Delta$ K280]. b, mRNA levels of genes affected by Kaem and Rhap in the hTau[F3 $\Delta$ K280] worms. c, Effects of pink-1, pdr-1, dct-1, sqst-1, and bec-1 on Kaem- and Rhap-induced associative memory in the $\mathrm{hTau}[\mathrm{F} 3 \Delta \mathrm{K} 280]$ worms. $\mathbf{d}$, A summary of mitophagy genes involved in Kaem and Rhap-induced memory improvement in the hTau[F3 $\Delta$ K280] worms. e, Memory assay of worms maintained on UV-killed OP50 treated with $0.2 \mathrm{mM} \mathrm{Kaem,} 0.2 \mathrm{mM}$ Rhap or Veh. f, Pharyngeal pumping rates at days 1, 5 and 9 of adulthood in worms treated with $0.2 \mathrm{mM} \mathrm{Kaem,} 0.2 \mathrm{mM}$ Rhap or Veh $(n=15)$. g, Mobility of hTau[P301L] worms treated with Kaem ( $n=50-100$ worms) or Rhap ( $n=50-100$ worms). Adult Day-1 worms were used. One set of data are shown from a total of two biological replicates. $\mathbf{h}-\mathbf{k}$, Effects of Kaem and Rhap on reduction of aggregated Tau in the HEK293 P301S Tau-Venus cells. Exogenous recombinant heparin-assembled P301S Tau (Tau seeds) induces conversion of endogenous Tau Venus from a dispersed distribution to bright foci $(h, i)$. Tests to determine the mechanism of the effect of drugs affecting the degradation of aggregated Tau were performed $(\mathbf{j}, \mathbf{k})$. One image/well was taken, with a total of 8 technical repeats per biological replicate. Data were analysed using ImageJ with images from 3 biological replicates. I, Toxicity evaluation of Kaem or Rhap on PC12 cells using MTT. All quantitative data are shown in mean \pm S.E.M. Two-way ANOVA followed by Tukey's multiple comparisons test (a-c, $\mathbf{e}-\mathbf{g}$ ), one-way ANOVA followed by Šidák's multiple comparisons test $(\mathbf{j}-\mathbf{I})$, and student $t$-test (i) were used for data analysis. NS, no significance, ${ }^{\star} p<0.05,{ }^{\star \star} p<0.01,{ }^{\star \star \star} p<0.001$. 
a

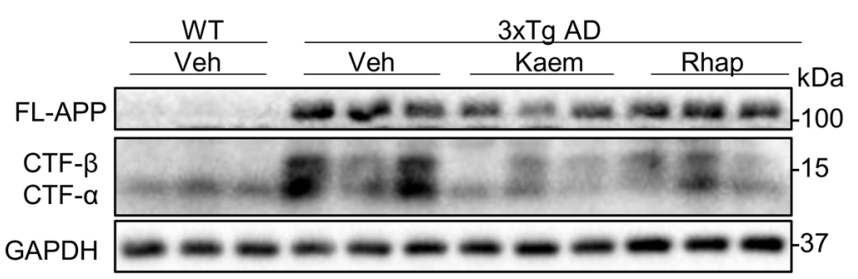

b

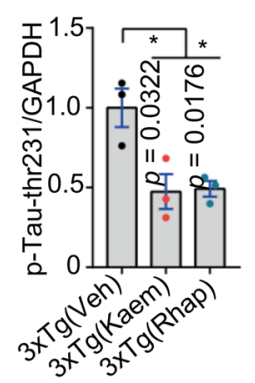

C
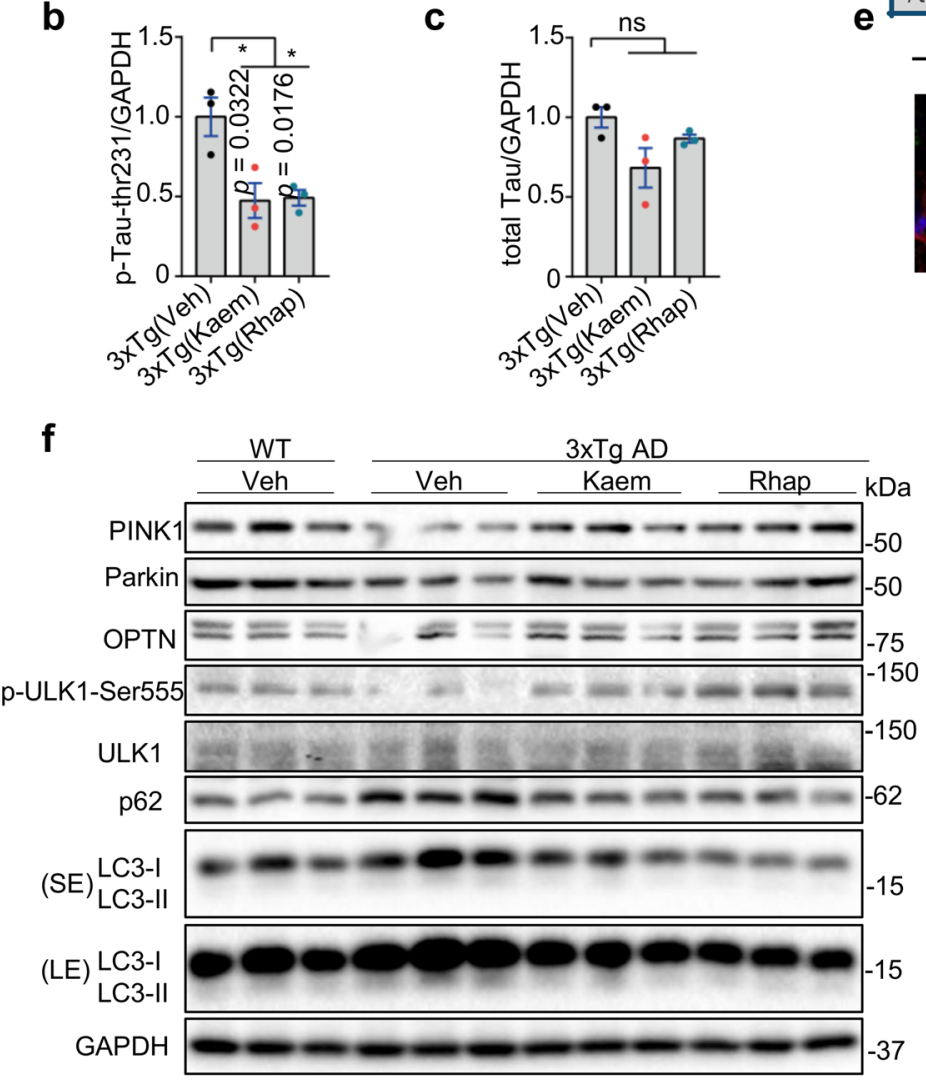

m
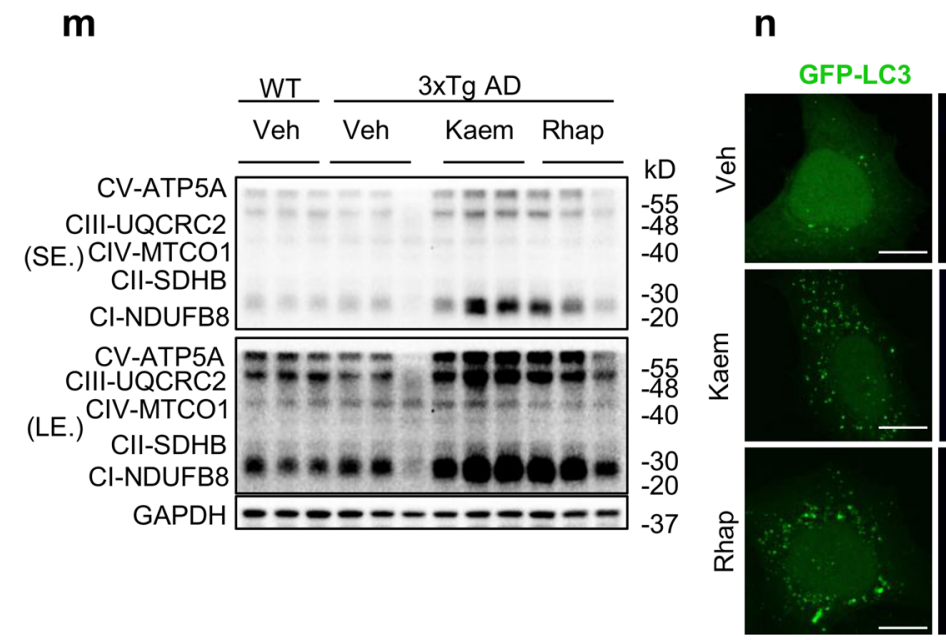

d

\begin{tabular}{|c|c|c|c|}
\hline \multicolumn{4}{|c|}{$3 x T g$ AD mice (compared with veh) } \\
\hline Parameters & Veh & Kaem & Rhap \\
\hline $\begin{array}{c}\text { Number of } \\
\text { microglia/ROI }\end{array}$ & $8.34 \pm 0.88$ & $15.67 \pm 0.66^{* *}$ & $13.67 \pm 1.20^{*}$ \\
\hline $\begin{array}{c}\text { Processes/microglia } \\
\text { (\% of Veh) }\end{array}$ & $100 \pm 5.26$ & $57.89 \pm 13.92^{*}$ & $63.16 \pm 9.12^{*}$ \\
\hline $\begin{array}{c}\text { Avg. process length } \\
\text { (\% of Veh) }\end{array}$ & $100 \pm 7.15$ & $64.86 \pm 8.11^{*}$ & $59.46 \pm 9.74^{*}$ \\
\hline $\begin{array}{c}\text { Microglia with } A \beta \\
\text { /total microglia (\%) }\end{array}$ & $35.36 \pm 3.47$ & $83.01 \pm 5.78^{* *}$ & $72.6 \pm 5.82^{* *}$ \\
\hline
\end{tabular}
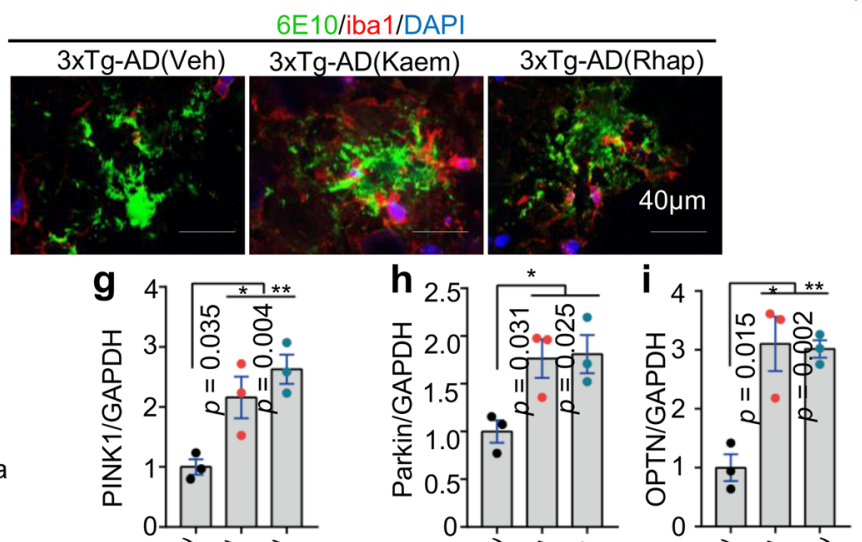
तentarinar
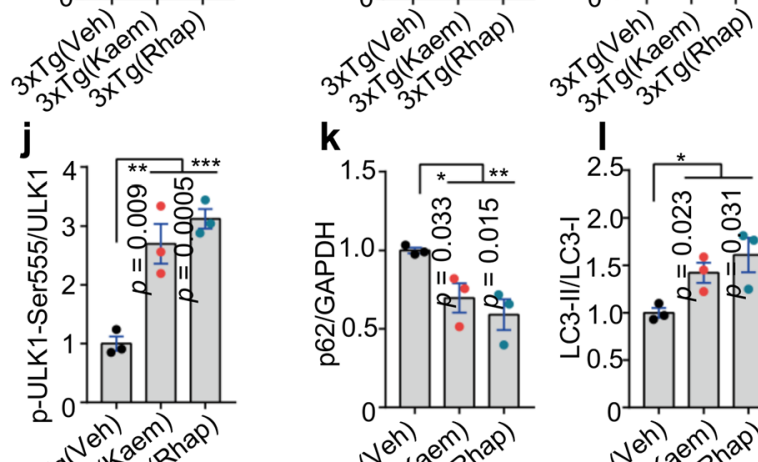

k
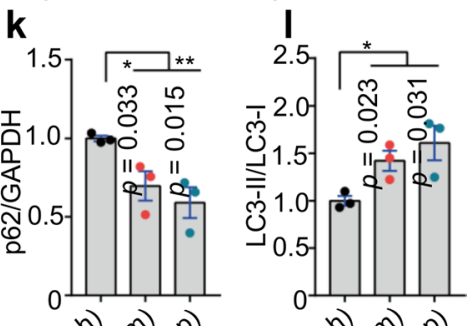

$3+3+3 x+3<0$

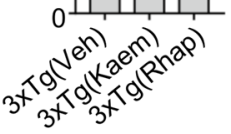

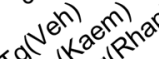

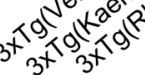

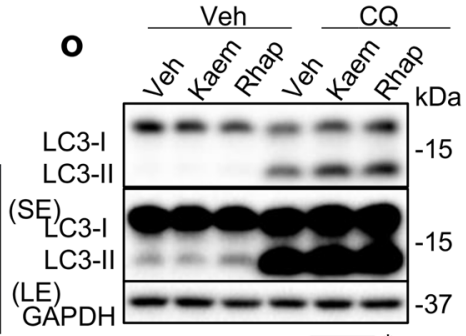
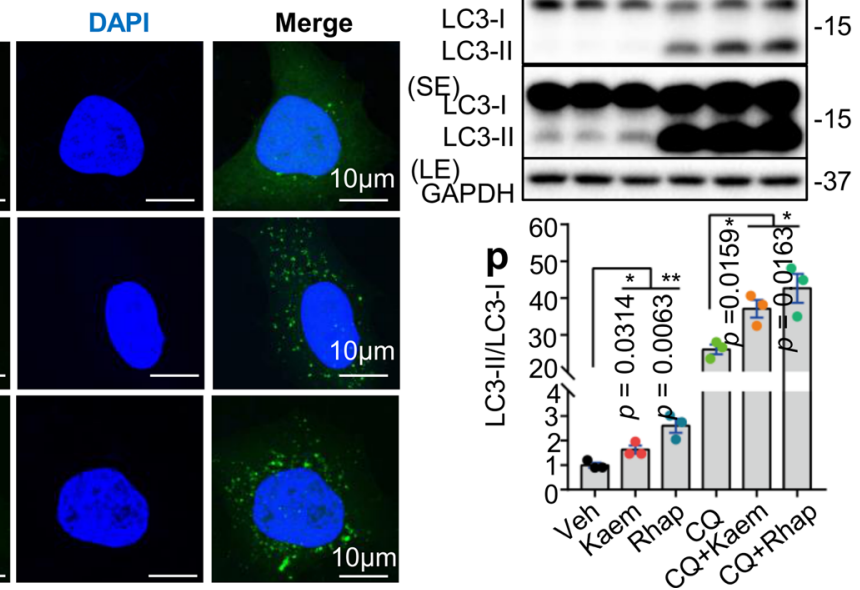

Extended Data Fig. 4 | See next page for caption. 
Extended Data Fig. 4 | Kaem and Rhap inhibit A $\beta$ production, increase microglial phagocytosis, and induce mitophagy and autophagy. a, Effects of Kaem and Rhap on protein levels of full-length APP (FL-APP), CTF- $\alpha$ and CTF- $\beta$ in hippocampal tissues from $3 x \operatorname{Tg}$ AD mice ( $n=3$ per group).

b, c, Quantification of phosphorylated Tau sites (Thr231) and total Tau/GAPDH in hippocampal tissues from 3xTg AD mice ( $n=3$ biologically independent samples). d, e, Effects of Kaem and Rhap on microglial phagocytosis of A $\beta$ plaques in hippocampal tissues from 3xTg AD mice. Data were quantified from 3 random images/mouse from a total of 3 mice (d). A $\beta$ plaques are shown in green (6E10 antibody) and microglia (anti-lba1 antibody) are in red (e). f, Western blot results showing the effects of Kaem and Rhap on the levels of proteins involved in mitophagy (PINK1, Parkin, OPTN, p-ULK1-Ser555 and ULK1) and substrates of autophagy (p62 and LC3-II/I) in the hippocampal tissues of the mice ( $n=3$ mice per group). g-I, Quantification of Western blot data in (f), $n=3$ biologically independent samples. se: short-exposure; le: long-exposure. $\mathbf{m}$, Western blot results showing the effects of Kaem and Rhap on the levels of proteins involved in OXPHOS in the hippocampal tissues of the mice ( $n=3$ mice per group). $\mathbf{n}$, Effects of Kaem and Rhap on autophagy induction using a HeLa cell line stably expressing GFP-LC3 following Kaem (10 $\mu \mathrm{M})$ or Rhap $(10 \mu \mathrm{M})$ treatment for $12 \mathrm{~h}$ before imaging. $\mathbf{0}$, $\mathbf{p}$, Western blot data (o) with semi-quantification ( $p$ ) showing the effects of Kaem and Rhap on levels of LC3-II/I in the HEK293 cells ( $n=3$ biologically independent samples). All quantitative data shown in mean \pm S.E.M. One-way ANOVA followed by Šidák's multiple comparisons test (b-d, $\mathbf{g}-\mathbf{I}, \mathbf{p}$ ) was used for data analysis. NS, no significance, ${ }^{\star} p<0.05,{ }^{\star *} p<0.01,{ }^{* \star} p<0.001$. Original unprocessed western blots for $\mathbf{a}, \mathbf{f}, \mathbf{m}, \mathbf{o}$ are available in Source Data Figs. 2-4. 
a

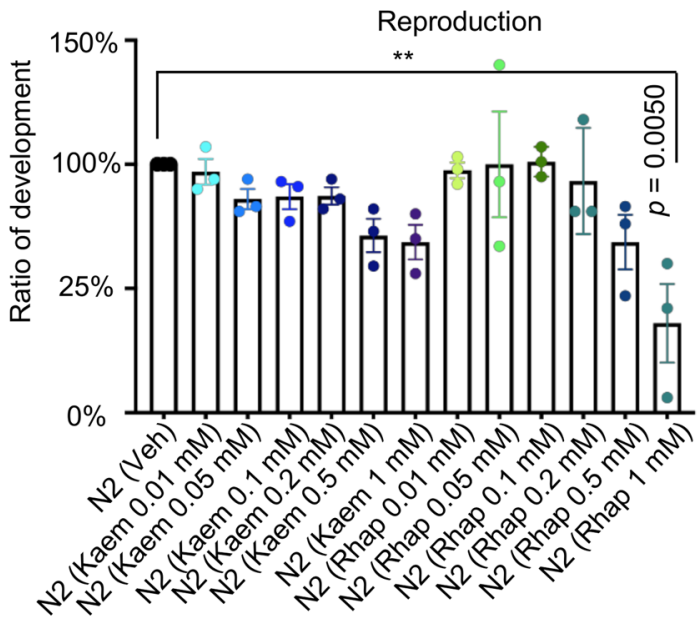

C

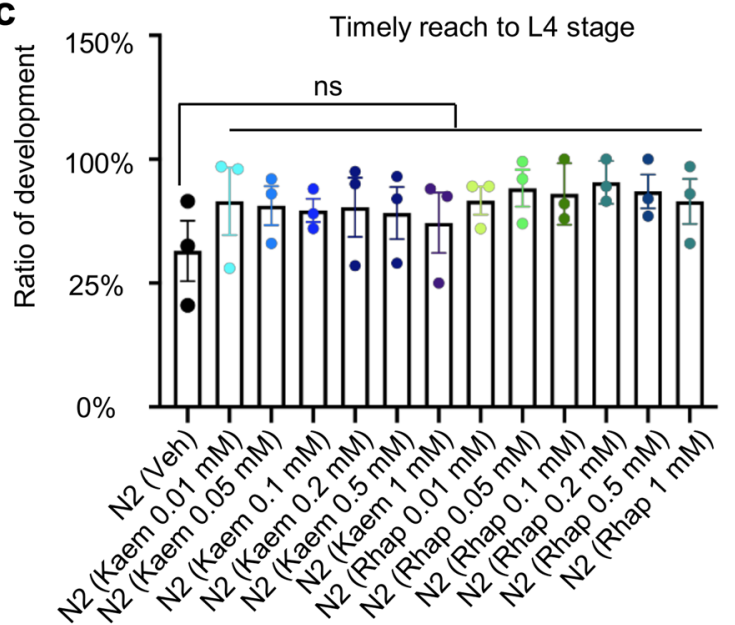

e
- Kaem 0.1 mM (Naive)

- Kaem 0.1 mM (IA)

- Kaem 0.2 mM (Naive)

- Kaem 0.2 mM (IA) Rhap $0.01 \mathrm{mM}$ (Naive) Rhap $0.01 \mathrm{mM}$ (IA) b
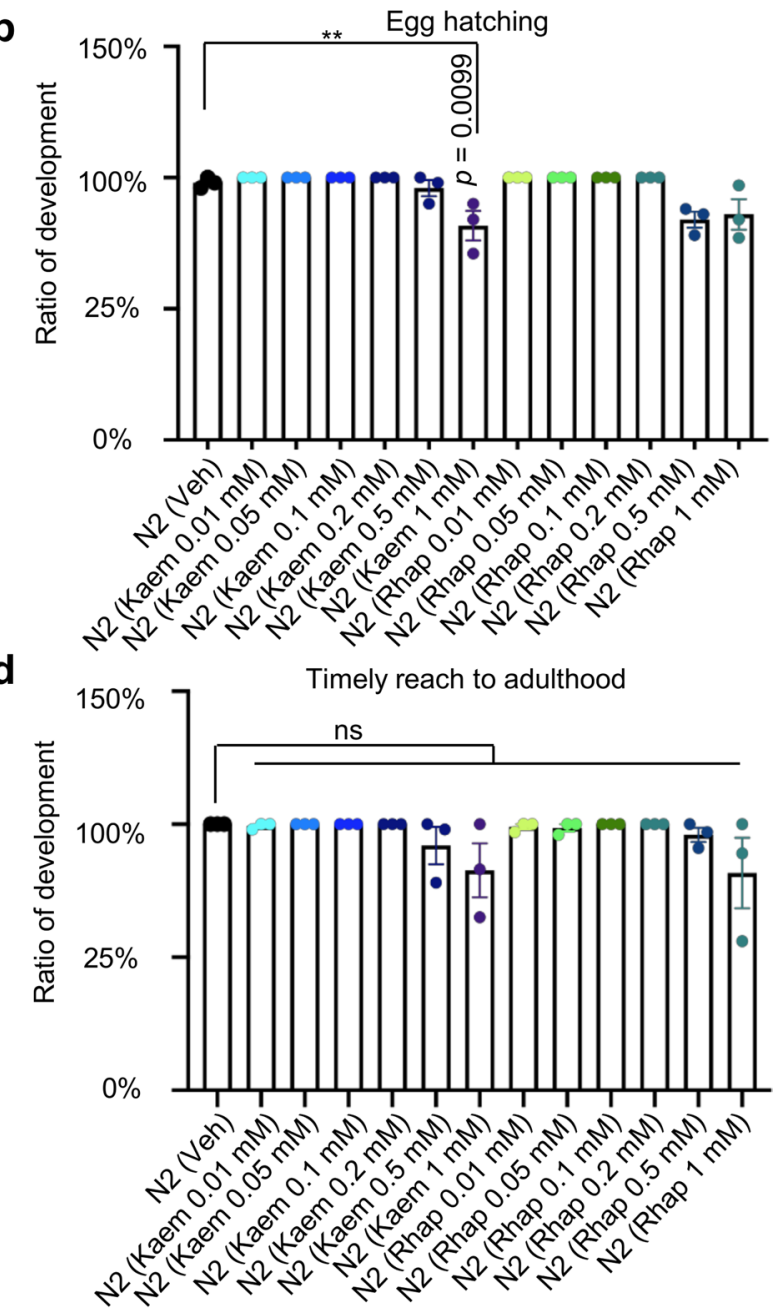

- Rhap 0.05 mM (Naive)

- Rhap 0.05 mM (IA)

- Rhap $0.1 \mathrm{mM}$ (Naive)

- Rhap 0.1 mM (IA)

- Rhap 0.2 mM (Naive)

- Rhap 0.2 mM (IA)

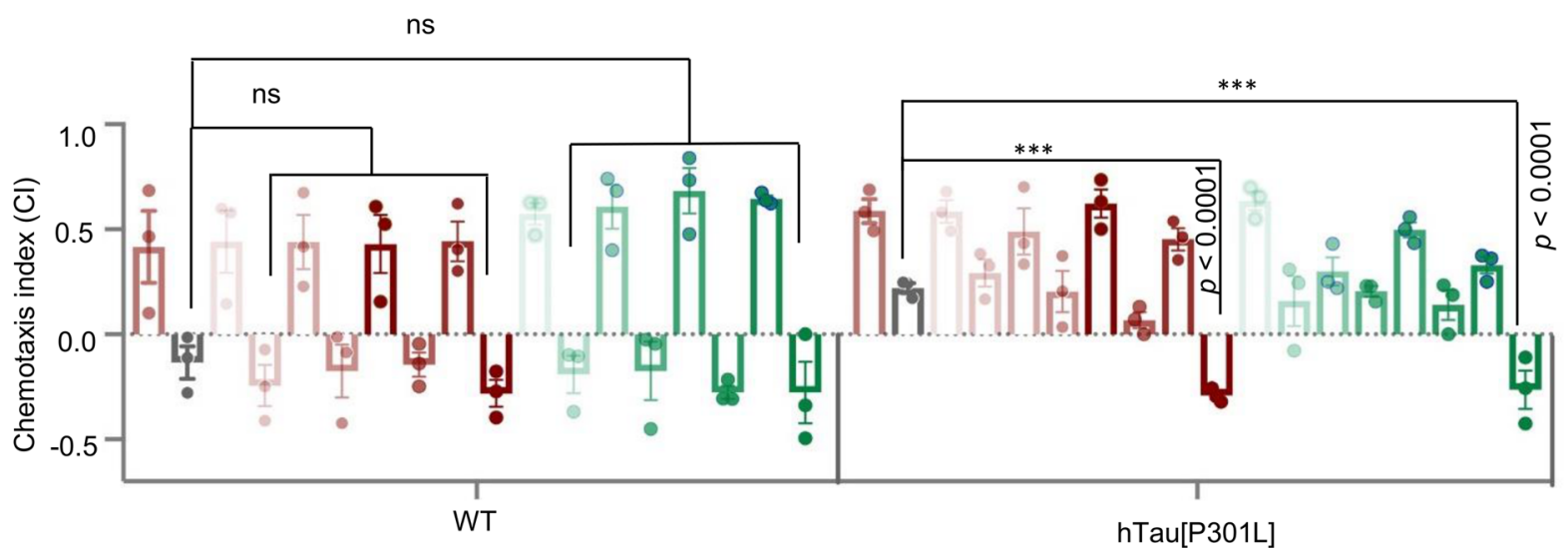

Extended Data Fig. 5 | See next page for caption. 
Extended Data Fig. 5 | Kaem and Rhap are toxicologically safe to nematodes up to $1 \mathrm{mM}$ and they do not show memory retention activity until reaching to $0.2 \mathrm{mM}$. Toxicological effects of Kaem or Rhap $(0.01,0.05,0.1,0.2 \mathrm{mM}$ from egg hatching onwards) on fecundity (3-h egg-lay) (a), egg hatching (b), development to L4 (c), and time to reach adulthood (d). e, Effects of different doses of Kaem and Rhap (0.01, 0.05, 0.1, 0.2 mM from egg hatching onwards) on associative memory in transgenic animals expressing hTau[301L]. All quantitative data are shown in mean \pm S.E.M. from three biological repeats. Two-way ANOVA followed by Tukey's multiple comparisons test was used for data analysis. NS, no significance, ${ }^{\star \star \star} p<0.001$. 


\section{Reporting Summary}

Nature Portfolio wishes to improve the reproducibility of the work that we publish. This form provides structure for consistency and transparency in reporting. For further information on Nature Portfolio policies, see our Editorial Policies and the Editorial Policy Checklist.

\section{Statistics}

For all statistical analyses, confirm that the following items are present in the figure legend, table legend, main text, or Methods section.

n/a Confirmed

$\searrow$ The exact sample size $(n)$ for each experimental group/condition, given as a discrete number and unit of measurement

$\searrow$ A statement on whether measurements were taken from distinct samples or whether the same sample was measured repeatedly

The statistical test(s) used AND whether they are one- or two-sided

Only common tests should be described solely by name; describe more complex techniques in the Methods section.

Х $\square$ A description of all covariates tested

Х $\square$ A description of any assumptions or corrections, such as tests of normality and adjustment for multiple comparisons

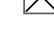

A full description of the statistical parameters including central tendency (e.g. means) or other basic estimates (e.g. regression coefficient)

AND variation (e.g. standard deviation) or associated estimates of uncertainty (e.g. confidence intervals)

For null hypothesis testing, the test statistic (e.g. $F, t, r$ ) with confidence intervals, effect sizes, degrees of freedom and $P$ value noted

Give $P$ values as exact values whenever suitable.

Х $\square$ For Bayesian analysis, information on the choice of priors and Markov chain Monte Carlo settings

Х $\square$ For hierarchical and complex designs, identification of the appropriate level for tests and full reporting of outcomes

$\square$ Estimates of effect sizes (e.g. Cohen's $d$, Pearson's $r$ ), indicating how they were calculated

Our web collection on statistics for biologists contains articles on many of the points above.

\section{Software and code}

Policy information about availability of computer code

Data collection

RDkit-2019 was used for molecule operation and similarity calculation. Scikit-learn 0.23 was used for data processing and clustering. Custom code was specific to our computing infrastructure and mainly used for data input/output and parallelization across computers and graphics processors. The code, currently under a patent-examination process, is available from the corresponding author on reasonable request.

Confocal Labmaze was used for behavioural tracking in the mouse studies.

Nikon, ZEN (blue edition) and Leica TCS SP8 confocal Laser Scanning Microscope System software were used for taking images.

Data analysis Microsoft Excel (2016) and Graphpad Prism 8 were used for data analysis. ImageJ with the plug in ObjectJ was used for the quantification of mitophagy levels.

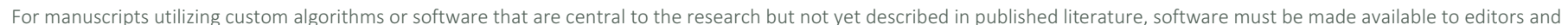

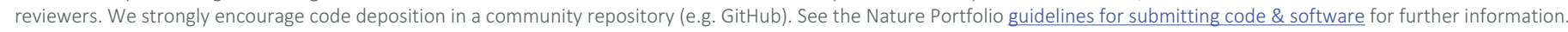

\section{Data}

Policy information about availability of data

All manuscripts must include a data availability statement. This statement should provide the following information, where applicable:

- Accession codes, unique identifiers, or web links for publicly available datasets

- A description of any restrictions on data availability

- For clinical datasets or third party data, please ensure that the statement adheres to our policy 


\section{Field-specific reporting}

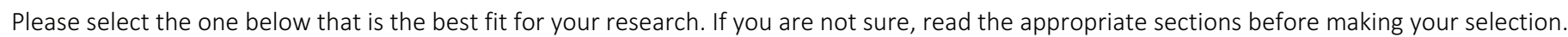

\section{Life sciences study design}

All studies must disclose on these points even when the disclosure is negative.

Sample size

Sample sizes for the behavioral tests were determined by the current standard used for mice in behavioral experiments, as well as based on the minimal amount of mice required to detect statistical significance with an alpha rate set at 0.05 in a standardly powered experiment. Thus, a total 6 mice/group were used for behavioral tests. The sample sizes used for immuno-histochemistry, Western blotting and other analyses were 3-6, as specified in the figure captions. Sample sizes for the C. elegans studies were 200-400 worms/group for the memory assay and around 100 worms/group for the lifespan studies. The sample sizes used in other experiments are detailed in the respective figure captions.

Data exclusions No data were excluded from analysis.

Replication

All the cell-culture and C. elegans experiments were performed with 3 biological repeats (with 3 technical repeats within each biological repeat), unless otherwise specified. All experiments were replicated successfully, with consistent data. The mouse data were from one biological experiment, as is common in the field.

Randomization Animals and samples were assigned randomly to the various experimental groups. Mice were randomly selected for the behavioural experiments.

Blinding

In data collection and analysis (mouse behavioural studies, mouse imaging-data analysis, and the imaging and data analysis of electron microscopy), the investigators were blinded to the experimental design.

\section{Reporting for specific materials, systems and methods}

We require information from authors about some types of materials, experimental systems and methods used in many studies. Here, indicate whether each material, system or method listed is relevant to your study. If you are not sure if a list item applies to your research, read the appropriate section before selecting a response.

\begin{tabular}{l|l} 
Materials \& experimental syste \\
\hline$n / a$ & Involved in the study \\
\hline & $\bigotimes$ Antibodies \\
$\square$ & $\bigotimes$ Eukaryotic cell lines \\
$\square$ & $\square$ Palaeontology and archaeology \\
$\square$ & $\square$ Animals and other organisms \\
$\square$ & $\square$ Clinical data \\
$\searrow$ & $\square$ Dual use research of concern
\end{tabular}

\begin{tabular}{l|l} 
Methods \\
\hline n/a & Involved in the study \\
$X$ & $\square$ ChIP-seq \\
$X$ & $\square$ Flow cytometry \\
$X$ & $\square$ MRI-based neuroimaging
\end{tabular}

\section{Antibodies}

Antibodies used

Antibodies used in western blot as follows (all from Cell Signaling Technology unless otherwise stated): PINK1 antibody (catalog no. ab75487, Abcam; no. A7131, ABclonal); Parkin antibody (no.NB100-91921; Novus); FUNDC1 antibody (no. ab74834; Abcam); LC3B antibody (no.NB100-2220; Novus); Beclin1 antibody (no.3495s); phospho-DRP1 antibody (no.S616); DRP1 antibody (no.8570s); p62 antibody (no.8025s); MFN2 antibody (no.94823s); phospho-ULK1 antibody (no.5869s); ULK1 antibody (no.6439s); AMBRA1 antibody (no.24907s); OPTN antibody (no. A1845, ABclona); Tau antibody (no. 46687s); p-Tau-thr181 (no. 12285s); p-Tau-thr231 (no. ab151559, Abcam); p-Tau-ser202/thr205 (no. MN1020, ThermoFisher Scientific); p-Tau-thr217 (no. 44-744, ThermoFisher Scientific); beta Amyloid polyclonal antibody (no. 51-2700, ThermoFisher Scientific); $\beta$-actin antibody (no. A5441; Sigma). Secondary antibodies including anti-mouse immunoglobulin G (IgG; catalog no. 7076s) and anti-rabbit IgG (no. 7074s). Specific primary antibodies used in immunohistochemistry include: mouse anti-B-Amyloid, 1-16 antibody (clone 6E10, catalog no. 803002; BioLegend); and rabbit anti-iba1 antibody (019-19741; Wako); mouse anti-AT8 antibody (cat no. MN1020; ThermoFisher Scientific). For first antibodies, the dilution ratio was 1:1000 or otherwise as specified else where; for secondary antibodies, the dilution ration was 1:5000 or otherwise as specified else where. 


\section{Eukaryotic cell lines}

Policy information about cell lines

Cell line source(s)

Mt-Keima HeLa cells were used for mitophagy evaluation; HEK293 cells stably expressing the ON4R isoform of human Tau, bearing the P301S mutation with a C-terminal venus were used for Tau aggregation and degradation. N2a and GFP-LC3 HeLa cells were cultured for mechanistic studies.

Authentication

None of the cell lines used were authenticated.

Mycoplasma contamination

All cell lines tested negative for mycoplasma contamination.

Commonly misidentified lines

(See ICLAC register)

No commonly misidentified cell lines were used.

\section{Animals and other organisms}

Policy information about studies involving animals; ARRIVE guidelines recommended for reporting animal research

Laboratory animals

3xTg-AD mice were provided on a B6;129 genetic background (Stock No. 004807 B6;129-Tg(APPSwe,tauP301L)1Lfa Psen1tm1Mpm/ Mmjax; https://www.jax.org/strain/004807) by the Jackson's laboratory. C57BL/6J mice were used as WT control. All animals were housed in individually ventilated cages on standardized rodent bedding. All animals were housed under constant-light cycle $(12 \mathrm{~h}$ light/dark) with food and water provided. 12.5-month-old 3xTg-AD female mice were used for two-month drug treatments by oral gavage, followed by behavioural and biochemical studies.

Wild animals

The study did not involve wild animals.

Field-collected samples

The study did not involve samples collected from the field.

Ethics oversight

All animal care and experimental procedures were approved by the Committee on the Ethics of Animal Experiments of the University of Macau (UMARE-013-2019).

Note that full information on the approval of the study protocol must also be provided in the manuscript. 